\title{
Conditions for shock revival by neutrino heating in core-collapse supernovae
}

\author{
H.-Th. Janka \\ Max-Planck-Institut für Astrophysik, Karl-Schwarzschild-Straße 1, 85741 Garching, Germany
}

Received 30 August 2000 / Accepted 12 December 2000

\begin{abstract}
Energy deposition by neutrinos can rejuvenate the stalled bounce shock and can provide the energy for the supernova explosion of a massive star. This neutrino-heating mechanism, though investigated by numerical simulations and analytic studies, is not finally accepted or proven as the trigger of the explosion. Part of the problem is that different groups have obtained seemingly discrepant results, and the complexity of the hydrodynamic models often hampers a clear and simple interpretation of the results. This demands a deeper theoretical understanding of the requirements of a successful shock revival. A toy model is developed here for discussing the neutrino heating phase analytically. The neutron star atmosphere between the neutrinosphere and the supernova shock can well be considered to be in hydrostatic equilibrium, with a layer of net neutrino cooling below the gain radius and a layer of net neutrino heating above. Since the mass infall rate to the shock is in general different from the rate at which gas is advected into the neutron star, the mass in the gain layer varies with time. Moreover, the gain layer receives additional energy input by neutrinos emitted from the neutrinosphere and the cooling layer. Therefore the determination of the shock evolution requires a time-dependent treatment. To this end the hydrodynamical equations of continuity and energy are integrated over the volume of the gain layer to obtain conservation laws for the total mass and energy in this layer. The radius and velocity of the supernova shock can then be calculated from global properties of the gain layer as solutions of an initial value problem, which expresses the fact that the behavior of the shock is controlled by the cumulative effects of neutrino heating and mass accumulation in the gain layer. The described toy model produces steady-state accretion and mass outflow from the nascent neutron star as special cases. The approach is useful to illuminate the conditions that can lead to delayed explosions and in this sense supplements detailed numerical simulations. On grounds of the model developed here, a criterion is derived for the requirements of shock revival. It confirms the existence of a minimum neutrino luminosity that is needed for shock expansion, but also demonstrates the importance of a sufficiently large mass infall rate to the shock. If the neutrinospheric luminosity or accretion rate by the shock are too low, the shock is weakened because the gain layer loses more mass than is resupplied by inflow. On the other hand, very high infall rates damp the shock expansion and above some threshold, the development of positive total energy in the neutrino-heating layer is prevented. Time-dependent solutions for the evolution of the gain layer show that the total specific energy transferred to nucleons by neutrinos is limited by about $10^{52} \operatorname{erg} M_{\odot}^{-1}(\sim 5 \mathrm{MeV}$ per nucleon). This excludes the possibility of very energetic explosions by the neutrino-heating mechanism, because the typical mass in the gain layer is about $0.1 M_{\odot}$ and does not exceed a few tenths of a solar mass. The toy model also allows for a crude discussion of the global effects of convective energy transport in the neutrino-heating layer. Transfer of energy from the region of maximum heating to radii closer behind the shock mainly reduces the loss of energy by the inward flow of neutrino-heated matter through the gain radius.
\end{abstract}

Key words. supernovae: general - elementary particles: neutrinos - hydrodynamics - accretion

\section{Introduction}

Neutrinos dominate the energetics of core-collapse supernovae. Only about one percent or $\sim 10^{51} \mathrm{erg}$ of the gravitational binding energy released in the formation process

Send offprint requests to: H.-Th. Janka,

e-mail: thj@mpa-garching.mpg.de of the compact remnant, usually a neutron star, end up as kinetic energy of the expanding ejecta, whereas $99 \%$ of this energy are radiated away in neutrinos. Electron captures on protons and nuclei trigger the gravitational instability of the iron core of an evolved massive star, because the electron number and thus the pressure are reduced by the escape of electron neutrinos (see, e.g., 
Bruenn 1986a). Later the loss of energy by the diffusion of neutrinos and antineutrinos of all flavors drives the evolution of the nascent neutron star from a hot, inflated configuration to the compact and very dense final state (Burrows \& Lattimer 1986).

Colgate \& White (1966) were the first to suggest that neutrinos may also play a crucial role for the explosion by taking up the gravitational binding energy of the collapsing core and depositing it in the rest of the star. Subsequent improvements and more realistic treatments of the microphysics, like equation of state (EoS) and neutrino transport, have changed our modern picture of stellar core collapse dramatically compared to the pioneering simulations by Colgate \& White (1966). Because of the discovery of weak neutral currents and the corresponding importance of neutrino scattering off nucleons and nuclei, the forming neutron star was recognized to be highly opaque to neutrinos. Therefore the neutrino luminosities turned out to be too low, and the energy transfer rate by neutrinos not large enough to invert the infall of the surrounding gas into an explosion. For many years, hopes and efforts therefore concentrated on the prompt bounceshock mechanism: the energy given to the hydrodynamical shock wave in the moment of core bounce was thought to lead directly to the ejection of the stellar mantle and envelope. Detailed models, however, showed that the shock experiences such severe energy losses by photodisintegration of iron nuclei and additional neutrino emission, that its outward propagation stops still well inside the iron core (e.g., Bruenn 1985, 1989a,b, 1993; Baron \& Cooperstein 1990; Hillebrandt 1987; Myra et al. 1987, 1989).

Wilson (1985), however, discovered that neutrinos can indeed cause an explosion on a timescale much longer than previously thought. More than 100 milliseconds after core bounce the conditions for neutrino energy deposition have significantly improved (Bethe \& Wilson 1985), and the mass infall rate and thus the ram pressure of the shock have decreased, making an explosion at later times easier than right after bounce (Burrows \& Goshy 1993; Bethe 1995). Although Wilson et al. (1986) obtained such "delayed" explosions via the neutrino-heating mechanism, their simulations gave rather low explosion energies, and their successes could not be confirmed by independent models with supposedly superior treatment of the neutrino physics and EoS (Bruenn 1986b, 1989a,b). Later simulations by Wilson \& Mayle $(1988,1993)$ and Mayle \& Wilson (1988) included neutron-finger convection in the nascent neutron star, which boosts the neutrino luminosities and thus increases the neutrino heating and the explosion energy. But whether neutron-finger convection actually occurs in the hot neutron star, or Ledoux-type convection (Burrows 1987; Keil et al. 1996; Pons et al. 1999), or none (Bruenn et al. 1995; Mezzacappa et al. 1998a) seems to depend on the properties of the nuclear EoS and possibly also on the treatment of the neutrino physics.

More recently, multi-dimensional simulations showed that convective overturn in the region of net neutrino heating between shock and gain radius (that is the po- sition outside the neutrinosphere where neutrino cooling is balanced by neutrino heating; Bethe \& Wilson 1985) can aid the explosion (Herant et al. 1994; Janka \& Müller 1995, 1996; Burrows et al. 1995) and can produce successes even when spherically symmetric models fail. This "convective engine" (Herant et al. 1994) or "boiling" (Burrows et al. 1995) transports cool gas into the region of strongest heating while at the same time hot gas rises towards the shock. Both effects increase the efficiency of neutrino energy transfer, reduce the energy loss by the reemission of neutrinos from the heated gas, and raise the postshock pressure, thus leading to more favorable conditions for shock expansion. While the existence and importance of postshock convection is not questioned, simulations with the most advanced treatment of the neutrino transport applied to multi-dimensional supernova calculations so far (Mezzacappa et al. 1998b; Lichtenstadt et al. 1999) nourished doubts whether the effects of convection are sufficiently strong to cause explosions.

Therefore scepticism about the viability of the delayed explosion mechanism by neutrino heating still remains (Thompson 2000), and seems justified even more because of recent observations which indicate a possible connection between gamma-ray bursts and at least some supernovae (e.g., Galama et al. 1998; Bloom et al. 1999). If confirmed, this discovery would require to consider large energies and/or asphericities of the explosions (Iwamoto et al. 1998; Woosley et al. 1999; Höflich et al. 1999) which might be hard to explain by the neutrino-driven mechanism. Therefore, despite the fact that the observations are still far from being conclusive, theorists feel tempted to speculate about alternative ways to power stellar explosions, e.g., by invoking magnetically driven jets (Wang \& Wheeler 1998; Khokhlov et al. 1999). However, while we know about the crucial role of neutrinos, we have no observational evidence or convincing theoretical argument in support of a dynamically important strength of magnetic fields in combination with a significant degree of rotation in the iron cores of all massive stars. Rather than in ordinary core-collapse supernovae, jets and a magnetohydrodynamic mechanism may be at work in cases where the neutrino-driven mechanism definitely fails, e.g., for progenitor main sequence masses above about $25 M_{\odot}$. (Fryer 1999) and when a black hole forms at the center of a rapidly spinning massive star (MacFadyen \& Woosley 1999; MacFadyen et al. 1999).

When judging about the viability of the neutrinodriven mechanism, one must, however, keep in mind the enormous complexity of the problem. Because of this complexity a number of approximations and simplifications had to be made in even the currently most refined hydrodynamical calculations. Some of these deficiencies have probably disadvantageous consequences for the efficiency of neutrino energy deposition in the postshock layers. Until very recently, all published hydrodynamical models employed, for example, a still unsatisfactory treatment of the neutrino transport. Instead of solving the Boltzmann transport equation, they used flux-limited 
diffusion schemes, a fact which underestimates the neutrino heating above the gain radius and overestimates the energy loss by neutrino emission below it (Janka 1991a, 1992; Messer et al. 1998; Yamada et al. 1999). Moreover, multidimensional supernova simulations have so far not been able to resolve the convective processes inside the nascent neutron star, although cooling models of neutron stars show their potential importance (Burrows 1987; Keil et al. 1996; Pons et al. 1999). Even more, recent investigations (e.g., Raffelt \& Seckel 1995; Janka et al. 1996; Burrows \& Sawyer 1998, 1999; Reddy et al. 1998, 1999; Yamada 2000; Yamada \& Toki 2000, and references therein) suggest that neutrino interaction rates in hot nuclear matter are suppressed compared to the standard description used in the numerical codes. Both the latter effects imply that the neutrino luminosities from the postcollapse core are most likely underestimated in current supernova models.

The neutrino-driven mechanism is by its nature sensitive to the neutrino-matter coupling in the heating region, which depends on the properties, i.e., spectra and luminosities, of the neutrino emission from the neutrinosphere and on the angular distribution of the neutrinos exterior to the neutrinosphere (Messer et al. 1998; Yamada et al. 1999; Burrows et al. 2000). These issues require not only the best possible technical treatment of the neutrino transport (cf. Mezzacappa et al. 2000; Liebendörfer et al. 2000; Rampp \& Janka 2000) and of the description of the neutrino opacities, but they can vary with the structure of the progenitor star, with general relativity, and with the nuclear EoS and therefore the compactness of the nascent neutron star. Differences of the simulations by different groups may be associated with one or more of these issues. Unfortunately, a detailed analysis and direct comparison is essentially impossible because of largely different numerical approaches and a complicated interdependence of effects.

In this unclear and extremely unsatisfactory situation a better fundamental understanding of the conditions and requirements for shock revival by neutrino heating is highly desirable. Several attempts were made for a discussion by analytic means (Bruenn 1993; Bethe 1993, 1995, 1997; Shigeyama 1995; Thompson 2000) or on grounds of simplified numerical analysis (Burrows \& Goshy 1993). While each of them contains interesting aspects and can shed light on certain results of simulations, they have led to contradictory conclusions, and none is general enough to be finally convincing. For example, assuming steadystate conditions (Burrows \& Goshy 1993) cannot explain how accretion is reversed into expansion, and why an accretion shock should contract again after moving outward for some while, a possibility which was in fact observed in many hydrodynamical simulations. The beginning of the reexpansion of the stalled shock and the phase when most of the explosion energy is deposited can also not be described by a stationary neutrino-driven baryonic wind (Qian \& Woosley 1996). Bethe (1990, 1993, 1995, 1997) gave a very useful and detailed discussion of the physics of neutrino heating, the structure and composition of the heating region, and the shock energetics and nucleosynthesis, using observational constraints from Supernova 1987A and numerical results provided mainly by Jim Wilson. Although addressing the question of the start of the shock, his analysis does not really reveal the requirements for a successful shock revival. Moreover, aspects were disregarded which have been recognized to be important for the outcome of simulations, for example the fact that rapid neutrino losses in the cooling region can weaken or even prevent an explosion (Woosley \& Weaver 1994; Janka \& Müller 1996; Messer et al. 1998). Bethe arrived at the conclusion that the explosion energy is delivered by neutrinos, whereas Bruenn (1993) and Thompson (2000) argued that neutrino heating is insufficient to cause an explosion because the advection timescale of the gas between shock and gain radius is too short for large energy deposition. Shigeyama (1995), on the other hand, performed a quasistationary analysis by expanding the physical variables in a power series of a small parameter, but his approach obscures the essential physics of shock revival rather than illuminating them.

The work presented here is a new approach for an analytic discussion of the conditions which can lead to the reexpansion of the supernova shock. The analysis is based on a simplified model for the post-bounce structure of the collapsed stellar core and generalizes the treatment of neutron star accretion by Chevalier (1989; see also Brown \& Weingartner 1994; Fryer et al. 1996). It is not meant to yield quantitative results or to be able to compete with detailed hydrodynamical simulations, but it should allow one to reproduce the basic features of the shock stagnation, accretion, and shock revival phases. It is therefore a supplementary tool which helps one getting a qualitative understanding of the processes that determine the postbounce evolution of the collapsed stellar core. In particular, the relative strength of competing effects that play a role in the neutrino-heating mechanism and their influence on the behavior of the supernova shock, i.e., its radial position and velocity as a function of time, can be estimated. This should help explaining why some models fail to produce explosions while others succeed.

The paper is organized in the following way. In Sect. 2 the physics of the post-bounce accretion phase will be described, in Sect. 3 the basic equations and corresponding assumptions used in the simplified analytic model will be introduced, in Sect. 4 the characteristic radii of the problem and their properties will be formally defined, in Sect. 5 the structure of the collapsed stellar core behind the stalled supernova shock will be discussed, in Sect. 6 expressions for the neutrino heating and cooling will be derived, in Sect. 7 the mass accretion rate of the nascent neutron star will be estimated, and in Sect. 8 the equations of mass and energy conservation will be applied to the neutrino heating layer, which leads to a criterion for the revival of a stalled supernova shock in Sect. 9. The equations derived in this paper will then be combined to an analytic toy model which allows one to integrate the 


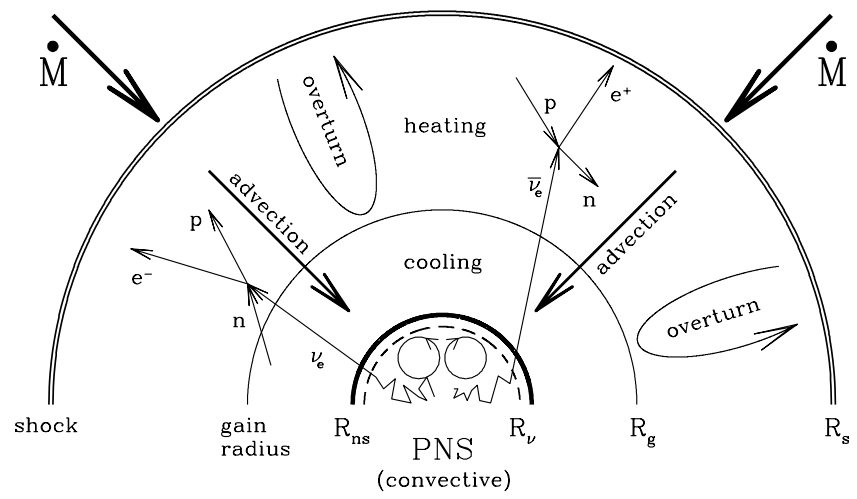

Fig. 1. Sketch which summarizes the processes that determine the evolution of the stalled supernova shock after core bounce. Stellar matter falls into the shock at radius $R_{\mathrm{s}}$ with a mass accretion rate $\dot{M}$ and a velocity near free fall. After deceleration in the shock, the gas is much more slowly advected towards the nascent neutron star through the regions of net neutrino heating and cooling, respectively. The radius $R_{\mathrm{ns}}$ of the neutron star is defined by a steep decline of the density over several orders of magnitude outside the neutrinosphere at $R_{\nu}$. Heating balances cooling at the gain radius $R_{\mathrm{g}}$. The dominant processes of energy deposition and loss are absorption of electron neutrinos onto neutrons and electron antineutrinos onto protons as indicated in the figure. Convective overturn mixes the layer between gain radius and shock, and convection inside the neutron star helps the explosion by boosting the neutrino luminosities

shock position, shock radius, and properties of the gain layer as functions of time by solving an initial value problem. A summary and conclusions will follow in Sect. 10.

\section{Physical picture}

Right after core bounce the hydrodynamic shock propagates outward in mass as well as in radius, being strongly damped by energy losses due to the photodisintegration of iron-group nuclei and neutrinos. The neutrino emission rises significantly when the shock breaks out into the neutrino-transparent regime. As a consequence, the pressure behind the shock is reduced and the velocities of the shock and of the fluid behind the shock, both of which were positive initially, decrease. Finally, the outward expansion of the shock stagnates, and the shock transforms into a standing accretion shock with negative gas velocity in the postshock region. The gas of the progenitor star, which continues to fall into the shock at a velocity near free fall, is decelerated abruptly within the shock. Below the shock it moves much more slowly towards the center, where it settles onto the surface of the nascent neutron star.

Figure 1 displays the most important physical elements which determine this evolutionary stage. Around the neutrinosphere at radius $R_{\nu}$, which is close to the radius $R_{\mathrm{ns}}$ of the proto-neutron star (PNS), the hot and comparatively dense gas loses energy by radiating neutrinos. If this energy sink were absent, the gas that is accreted through the shock at a rate $\dot{M}$ would pile up in a growing, high-entropy atmosphere on top of the compact remnant (Colgate et al. 1993; Colgate \& Fryer 1995; Fryer et al. 1996). But since neutrinos are emitted efficiently at the thermodynamical conditions around the neutrinosphere, the entropy of the gas is reduced so that the gas can be absorbed into the surface of the neutron star. The mass flow through the neutrinospheric region is therefore triggered by the neutrino energy loss and allows more gas to be advected inward from larger radii. In case of stationary accretion the temperature at the base of the atmosphere ensures that the emitted neutrinos carry away the gravitational binding energy of the matter which is added to the neutron star at a given accretion rate. In fact, this requirement closes the set of equations that determines the steady state of the accretion system and allows one to determine the radius $R_{\mathrm{S}}$ of the accretion shock (see, e.g., Chevalier 1989; Brown \& Weingartner 1994; Fryer et al. 1996).

At the so-called gain radius $R_{\mathrm{g}}$ (Bethe \& Wilson 1985) between neutrinosphere $R_{\nu}$ and shock position $R_{\mathrm{s}}$, the temperature of the atmosphere becomes so low that the absorption of high-energy electron neutrinos and antineutrinos starts to exceed the neutrino emission. This radius therefore separates the region of net neutrino cooling below from a layer of net heating above. Since the neutrino heating is strongest just outside the gain radius and the propagation of the shock has weakened before stagnation, a negative entropy gradient is built up in the postshock region. This leads to convective overturn roughly between $R_{\mathrm{g}}$ and $R_{\mathrm{s}}$, which transports hot matter outward in rising high-entropy bubbles. At the same time cooler material is mixed inward in narrow, low-entropy downflows (Herant et al. 1994; Burrows et al. 1995; Janka \& Müller 1996). Inside the nascent neutron star, below the neutrinosphere, convective motions can enhance the neutrino emission by carrying energy faster to the surface than neutrino diffusion does (Keil et al. 1996).

Between neutrinosphere and the supernova shock a number of approximations apply to a high degree of accuracy, which help one developing a simple analytic understanding of the effects that influence the evolution of the supernova shock. Figure 2 shows schematically the profiles of density, temperature and mass accretion rate in that region. A formal discussion follows in the subsequent sections. Outside the neutrinosphere (typically at about $10^{11} \mathrm{~g} / \mathrm{cm}^{3}$ ) the temperature drops slowly compared to the density decline, which is steep. When nonrelativistic nucleons dominate the pressure, the decrease of the density yields the pressure gradient which ensures hydrostatic equilibrium in the gravitational field of the neutron star. Assuming a temperature equal to the neutrinospheric temperature in this region is a reasonably good approximation for the following reasons. On the one hand, the cooling rate depends sensitively both on density and temperature, and the density drops rapidly. Therefore the total energy loss is determined in the immediate vicinity of the neutrinosphere and the details of the temperature profile do not matter very much. On the other hand, efficient neutrino heating prevents that the temperature can drop much 

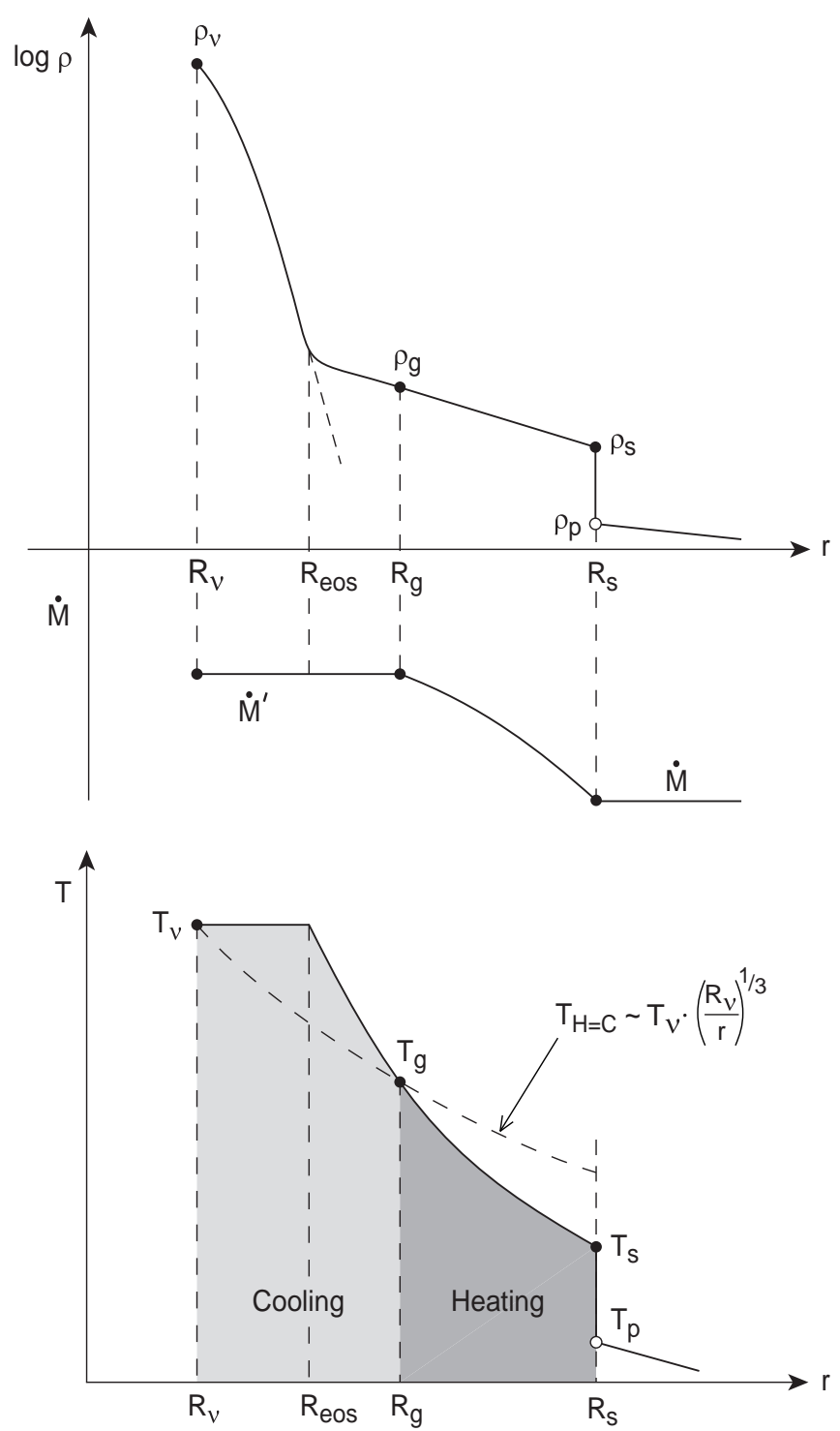

Fig. 2. Schematic profiles of density, temperature, and mass accretion rate between neutrinosphere at radius $R_{\nu}$ and shock at $R_{\mathrm{s}}$ some time after core bounce. $R_{\mathrm{g}}$ denotes the position of the gain radius. At the shock, $\rho$ and $T$ jump discontinuously from their preshock values $\rho_{\mathrm{p}}$ and $T_{\mathrm{p}}$ to the postshock values $\rho_{\mathrm{s}}$ and $T_{\mathrm{s}}$, respectively. For $r<R_{\text {eos }}$ the density declines steeply because the pressure is mainly caused by the nonrelativistic Boltzmann gases of free neutrons and protons. Outside of $R_{\text {eos }}$ the gas is radiation dominated and the density decrease much flatter. In general, some of the gas falling into the shock at rate $\dot{M}$ may stay in the region of neutrino heating while another part (rate $\dot{M}^{\prime}$ ) is advected into the nascent neutron star. Note that $\dot{M}(r)$ is continuous at the shock in the rest frame of the star only in case of a stalled shock front. Between $R_{\nu}$ and $R_{\text {eos }}$ the temperature can be considered roughly as constant, whereas its negative gradient in the radiation dominated region ensures hydrostatic equilibrium. There is net energy loss between $R_{\nu}$ and $R_{\mathrm{g}}$ where $T(r)$ exceeds the temperature $T_{\mathrm{H}=\mathrm{C}} \sim T_{\nu}\left(R_{\nu} / r\right)^{1 / 3}$, for which neutrino heating equals cooling. Net energy deposition occurs between $R_{\mathrm{g}}$ and $R_{\mathrm{s}}$

below the neutrinospheric value. If, instead, the temperature would rise significantly above this latter value, the matter would become optically thick to the energetic neutrinos produced in the hot gas (the opacity increases roughly with the square of the neutrino energy) and the neutrinosphere would move farther out to a lower density (and thus typically a lower temperature).

Below a density between $10^{9} \mathrm{~g} / \mathrm{cm}^{3}$ and $10^{10} \mathrm{~g} / \mathrm{cm}^{3}$, relativistic electron-positron pairs and radiation determine the pressure, provided the temperature is sufficiently high, typically around $1 \mathrm{MeV}$ or more (see Woosley et al. 1986). Exterior to the corresponding radius $R_{\text {eos }}$, where this transition from the baryon-dominated to the radiation-dominated regime takes place, the temperature must therefore decrease so that the negative temperature gradient can yield the force which balances gravity.

The gain radius $R_{\mathrm{g}}$ is located at the radial position where the temperature profile $T(r)$ intersects with the curve of temperature values, $T_{\mathrm{H}=\mathrm{C}}(r)$, for which heating is equal to cooling by neutrinos, roughly given by

$T_{\mathrm{H}=\mathrm{C}}(r) \sim T_{\nu} \cdot\left(\frac{R_{\nu}}{r}\right)^{\frac{1}{3}}$

(Bethe \& Wilson 1985). In Eq. (1) $T_{\nu}$ means the temperature at the radius $R_{\nu}$ of the neutrinosphere. The shock at $R_{\mathrm{s}}$ is taken to be infinitesimally thin compared to the scales considered. Within the shock the density and temperature therefore jump from their preshock values $\rho_{\mathrm{p}}$ and $T_{\mathrm{p}}$, to the postshock values $\rho_{\mathrm{s}}$ and $T_{\mathrm{s}}$, respectively. A part of the gas which falls into the shock with a mass accretion rate $\dot{M}$ can stay in the region of neutrino heating, whereas another part is advected with rate $\dot{M}^{\prime}$ through the cooling region to be added to the neutron star inside $R_{\nu}$.

The approach to the problem of shock revival taken in this paper is considerably different from the discussion of steady-state accretion or winds. Steady-state assumptions, for example, were also used by Burrows \& Goshy (1993) in their theoretical analysis of the explosion mechanism. Having realized the fact, however, that the mass and energy in the gain layer vary because of different rates of mass flow through the boundaries and additional neutrino heating, one is forced to the following conclusions. Firstly, the discussion has to be time-dependent, which means that the time derivatives in the continuity and energy equations cannot be ignored. (Dropping the total time-derivative in the momentum equation by assuming hydrostatic equilibrium is less problematic and yields a reasonably good approximation.) Secondly, the properties of the shock and of the gain layer must be determined as solutions of an initial value problem rather than from a steady-state picture. This reflects essential physics, namely that the shock behavior is controlled by the cumulative effects of neutrino heating and mass accumulation in the gain layer. For these reasons conservation laws for the total mass and energy in the gain layer will be derived by integrating the hydrodynamic equations of continuity and energy, including the terms with time derivatives, over the volume of the gain layer. The treatment will therefore retain the timedependence of the problem. 
In this paper the discussion will be restricted to an idealized, spherically symmetric situation and possible convective mixing will be assumed to lead to efficient homogenization of the unstable layer. Certainly, this is not a good assumption for the convective overturn that takes place in the region between gain radius and shock front, where prominent, large-scale inhomogeneities develop (Herant et al. 1994; Burrows et al. 1995; Janka \& Müller 1996). Bethe (1995) has made attempts to discuss the physical implications of the simultaneous presence of low-entropy downstreams and high-entropy rising bubbles. For this purpose he introduced free parameters, e.g., to quantify the fraction of neutrinos that hits the cold downflows and is effective for their heating, or to account for the part of the matter that is added to the neutron star instead of being pushed outward in the expanding bubbles. This procedure is not really satisfactory and will not be copied here. Instead, an admittedly simplified and idealized spherical situation will be considered to highlight the conditions needed for shock revival and to develop a qualitative understanding of the influence of different effects. One-dimensional analysis can help developing a better understanding of the delayed explosion mechanism, because simulations in spherical symmetry have produced successful explosions (Wilson 1985; Wilson et al. 1986; Janka \& Müller 1995, 1996). Thus they have demonstrated that convection behind the shock is not an indispensable requirement for an explosion, although it may be an essential (Herant et al. 1994; Burrows et al. 1995; Janka \& Müller 1996) - yet not necessarily sufficient (Janka \& Müller 1996; Mezzacappa et al. 1998b; Lichtenstadt et al. 1999) - ingredient to obtain explosions, or to raise the explosion energy in cases which fail or nearly fail in spherical symmetry.

\section{Basic equations and assumptions}

The hydrodynamic equations are considered in Eulerian form for spherical symmetry with source terms for Newtonian gravity and neutrino energy and momentum exchange with the stellar medium. The equations of continuity, momentum, and energy are:

$$
\begin{aligned}
& \frac{\partial \rho}{\partial t}+\frac{1}{r^{2}} \frac{\partial}{\partial r}\left(r^{2} \rho v\right)=0, \\
& \frac{\partial(\rho v)}{\partial t}+\frac{1}{r^{2}} \frac{\partial}{\partial r}\left(r^{2} \rho v^{2}\right)=-\frac{\partial P}{\partial r}-\rho \frac{\partial \Phi}{\partial r}, \\
& \frac{\partial e}{\partial t}+\frac{1}{r^{2}} \frac{\partial}{\partial r}\left[r^{2} v(e+P)\right]=-\rho v \frac{\partial \Phi}{\partial r}+Q_{\nu} .
\end{aligned}
$$

Here $r, v, \rho, P, t$ are radius, fluid velocity, density, pressure, and time, respectively, and $e$ is defined as the sum of internal energy density, $\varepsilon$, and kinetic energy density of the gas:

$e=\frac{1}{2} \rho v^{2}+\varepsilon$
The term $Q_{\nu}$ denotes the rate of energy gain or loss per unit volume by neutrino heating and cooling. $\Phi(r)$ is an effective potential which contains contributions from the gravitational potential and from the momentum transfer to the stellar gas by neutrinos. Neglecting self-gravity of the gas in the region between neutrinosphere and supernova shock, it can be written as

$\Phi=-\frac{G \widetilde{M}}{r}=-\frac{G}{r}\left(M-\frac{\left\langle\kappa_{t}\right\rangle L_{\nu}}{4 \pi G c \rho}\right)$

Here $G$ is the gravitational constant, $c$ the speed of light, $M$ the mass inside $R_{\nu}$ and $\widetilde{M}$ means an effective mass that includes the momentum transfer term and is defined by the term in brackets on the right side of Eq. (6). When self-gravity is disregarded, the mass of the gas between $R_{\nu}$ and $R_{\mathrm{s}}$ must be negligible compared to the neutron star mass $M$, i.e.,

$\Delta M=\int_{R_{\nu}}^{R_{\mathrm{s}}} \mathrm{d} r 4 \pi r^{2} \rho(r) \ll M$.

In Eq. (6) $L_{\nu}=\sum_{\nu_{i}} L_{\nu_{i}}$ is the total neutrino luminosity and $\left\langle\kappa_{\mathrm{t}}\right\rangle$ the mean total opacity calculated as an average of the total opacities of neutrinos $\nu_{i}$ and antineutrinos $\bar{\nu}_{i}$ of all flavors according to

$\left\langle\kappa_{\mathrm{t}}\right\rangle L_{\nu} \equiv \sum_{\nu_{i}} \kappa_{\mathrm{t}, \nu_{i}} L_{\nu_{i}}+\sum_{\bar{\nu}_{i}} \kappa_{\mathrm{t}, \bar{\nu}_{i}} L_{\bar{\nu}_{i}}$

The total opacity $\kappa_{\mathrm{t}, \nu_{i}}$ of neutrino $\nu_{i}$ is considered to be averaged over the spectrum of the corresponding energy flux. Note that in this paper, opacities are defined as inverse mean free paths and are thus measured in units of $1 / \mathrm{cm}$. Equations (3), (4), and (6) imply that the momentum transfer rate from neutrinos to the stellar gas is written as $\left\langle\kappa_{\mathrm{t}}\right\rangle L_{\nu} /\left(4 \pi c r^{2}\right)$ with $L_{\nu}$ and $\left\langle\kappa_{\mathrm{t}}\right\rangle / \rho$ not depending on $r$. This is approximately fulfilled in the optically thin regime for neutrinos, i.e., exterior to the neutrinosphere where the neutrino luminosities and spectra are roughly constant. Yet it is not exactly true, because the concept of "the" neutrinosphere is fuzzy and neutrino emission and absorption continue even outside the neutrinosphere. In addition, the opacity depends on the composition which varies with the radius. During all of the post-bounce evolution, however, the typical total neutrino luminosity is only a few per cent of the Eddington luminosity,

$L_{\mathrm{Edd}, \nu} \equiv \frac{4 \pi G M c \rho}{\left\langle\kappa_{\mathrm{t}}\right\rangle}$

Therefore the neutrino source terms for momentum in Eq. (3) and for kinetic energy in Eq. (4), which are carried by the potential $\Phi$, are always small and the approximate treatment following below is justified.

Neutrinos transfer momentum to the stellar medium by neutral-current scatterings off neutrons and protons. The corresponding transport opacity for these scattering processes is

$\kappa_{\mathrm{sc}} \approx \frac{5 \alpha^{2}+1}{24} \frac{\sigma_{0}\left\langle\epsilon_{\nu}^{2}\right\rangle}{\left(m_{\mathrm{e}} c^{2}\right)^{2}} \frac{\rho}{m_{\mathrm{u}}}\left(Y_{\mathrm{n}}+Y_{\mathrm{p}}\right)$. 
Here $m_{\mathrm{u}} \approx 1.6610^{-24} \mathrm{~g}$ is the atomic mass unit, $m_{\mathrm{e}} c^{2}=0.511 \mathrm{MeV}$ the rest-mass energy of the electron, $\sigma_{0}=1.7610^{-44} \mathrm{~cm}^{2}$, and $Y_{\mathrm{n}}=n_{\mathrm{n}} / n_{\mathrm{b}}$ and $Y_{\mathrm{p}}=n_{\mathrm{p}} / n_{\mathrm{b}}$ are the number fractions of free neutrons and protons, i.e., their particle densities normalized to the number density $n_{\mathrm{b}}$ of nucleons. A minor difference between the neutrinoproton and the neutrino-neutron scattering cross section due to different vector coupling constants is ignored, and also the axial-vector couplings are assumed to be the same and to be equal to the charged-current axial-vector coupling constant in vacuum, $\alpha=-1.26$. Additional scattering reactions with electrons and positrons can be neglected because of their much smaller cross sections, and neutrino scattering off nuclei is unimportant because the postbounce medium exterior to the neutrinosphere is nearly completely disintegrated into free nucleons.

In case of $\nu_{\mathrm{e}}$ and $\bar{\nu}_{\mathrm{e}}$ also the charged-current absorptions on neutrons and protons, respectively, need to be taken into account due to their large cross sections. The absorption opacity is

$\kappa_{\mathrm{a}} \approx \frac{3 \alpha^{2}+1}{4} \frac{\sigma_{0}\left\langle\epsilon_{\nu}^{2}\right\rangle}{\left(m_{\mathrm{e}} c^{2}\right)^{2}} \frac{\rho}{m_{\mathrm{u}}}\left\{\begin{array}{c}Y_{\mathrm{n}} \\ Y_{\mathrm{p}}\end{array}\right\}$.

In Eqs. (10) and (11) the recoil of the nucleon and phase space blocking effects for the fermions are neglected, which is very good at the conditions considered in this paper. In both neutral-current and charged-current processes only the leading terms depending on the squared neutrino energy, $\epsilon_{\nu}^{2}$, are taken into account. Averaging over the spectrum of the neutrino energy flux yields the factor $\left\langle\epsilon_{\nu}^{2}\right\rangle$, for which Eq. (25) provides a suitable definition, if minor differences between the spectra of neutrino energy density and flux density are disregarded.

The total opacity includes the contributions from scattering and absorption and is given as $\kappa_{\mathrm{t}, \nu_{i}}=\kappa_{\mathrm{sc}, \nu_{i}}+\kappa_{\mathrm{a}, \nu_{i}}$. With typical values $L_{\nu_{\mathrm{e}}}=L_{\bar{\nu}_{\mathrm{e}}}=L_{\nu_{x}}=\frac{1}{6} L_{\nu}$ (with $\nu_{x} \in\left\{\nu_{\mu}, \bar{\nu}_{\mu}, \nu_{\tau}, \bar{\nu}_{\tau}\right\}$ and $L_{\nu_{x}}$ being the luminosity of each individual type of $\left.\nu_{x}\right),\left\langle\epsilon_{\bar{\nu}_{\mathrm{e}}}^{2}\right\rangle \approx 2\left\langle\epsilon_{\nu_{\mathrm{e}}}^{2}\right\rangle$ and $\left\langle\epsilon_{\bar{\nu}_{x}}^{2}\right\rangle \approx 4\left\langle\epsilon_{\nu_{\mathrm{e}}}^{2}\right\rangle$, the total opacity averaged for all neutrinos and antineutrinos can be estimated from Eq. (8) as

$$
\begin{aligned}
\left\langle\kappa_{\mathrm{t}}\right\rangle & \approx \frac{113 \alpha^{2}+25}{144} \frac{\sigma_{0}\left\langle\epsilon_{\nu_{\mathrm{e}}}^{2}\right\rangle}{\left(m_{\mathrm{e}} c^{2}\right)^{2}} \frac{\rho}{m_{\mathrm{u}}}\left(Y_{\mathrm{n}}+Y_{\mathrm{p}}\right) \\
& \approx 1.910^{-7} \rho_{10}\left(\frac{k T_{\nu_{\mathrm{e}}}}{4 \mathrm{MeV}}\right)^{2}\left[\frac{1}{\mathrm{~cm}}\right] .
\end{aligned}
$$

For deriving the first expression, the factor $Y_{\mathrm{n}}+2 Y_{\mathrm{p}}$ in the absorption term was replaced by $Y_{\mathrm{n}}+Y_{\mathrm{p}}$. This is a reasonably good approximation because $Y_{\mathrm{p}} \lesssim Y_{\mathrm{n}}$ between the neutrinosphere and the shock, and the corresponding change of the absorption opacity of electron antineutrinos implies only a minor error in the total opacity. In the second equation use was made of $Y_{\mathrm{n}}+Y_{\mathrm{p}} \approx 1$. If the neutrino flux spectrum has Fermi-Dirac shape with vanishing degeneracy, the neutrino temperature $T_{\nu}$ is related to the mean squared neutrino energy by $\left\langle\epsilon_{\nu}^{2}\right\rangle \approx$ $21\left(k T_{\nu}\right)^{2} . k$ is the Boltzmann constant and $\rho_{10}$ the density measured in $10^{10} \mathrm{~g} / \mathrm{cm}^{3}$. For a total neutrino luminosity
$L_{\nu}=10^{53} \mathrm{erg} / \mathrm{s}$, neutrino momentum transfer reduces $\widetilde{M}$ in Eq. (6) relative to $M$ by about $3.810^{-2} M_{\odot}$, which is indeed a small correction.

\section{The characteristic radii}

The neutrinospheric radius $R_{\nu}$, the gain radius $R_{\mathrm{g}}$ and the transition radius of the EoS properties, $R_{\text {eos }}$, will be formally defined below. They are characteristic of the atmospheric structure in the postshock region, which determines, together with the infall region ahead of the shock, the shock radius $R_{\mathrm{s}}$ and the shock velocity $U_{\mathrm{s}} \equiv \dot{R}_{\mathrm{s}}$.

\subsection{The neutrinosphere}

The neutrinosphere relevant for the discussion in the following sections is the "energy-sphere", where neutrinos decouple energetically from the stellar background. It usually does not coincide with the sphere of last scattering, the so-called "transport-sphere", outside of which the neutrino distribution becomes strongly forward peaked (for a detailed discussion, see Janka 1995). Only inside their energy-sphere neutrinos can be considered to be roughly in thermodynamic equilibrium with the stellar medium. Besides neutrino-nucleon scattering, which is important for all neutrinos, electron neutrinos $\nu_{\mathrm{e}}$ and electron antineutrinos $\bar{\nu}_{\mathrm{e}}$ interact via frequent chargedcurrent absorption and emission reactions with nucleons, whereas muon and tau neutrinos and antineutrinos do not. Therefore the energy-spheres of electron neutrinos and antineutrinos are typically located farther out in the star at larger radii than those of muon and tau neutrinos.

The energy deposition in the gain region, however, is clearly dominated by $\nu_{\mathrm{e}}$ and $\bar{\nu}_{\mathrm{e}}$. For this reason one can concentrate on their transport properties and neglect muon and tau neutrinos and antineutrinos in the discussion. Scattering off nucleons acts on all neutrinos equally. The charged-current absorption reactions of $\nu_{\mathrm{e}}$ and $\bar{\nu}_{\mathrm{e}}$ on neutrons and protons, respectively, yield an even larger contribution to the total opacity. The opacities of $\nu_{\mathrm{e}}$ and $\bar{\nu}_{\mathrm{e}}$ are nearly equal, because $\bar{\nu}_{\mathrm{e}}$ absorption and emission (Eq. (18)) is similarly frequent as $\nu_{\mathrm{e}}$ absorption and emission (Eq. (17)) as long as positrons are abundant, i.e., the stellar atmosphere is hot and electrons are not very degenerate. Therefore the transport-spheres and energy-spheres of electron neutrinos and antineutrinos are all close together and it is justified to consider only one, "the", neutrinosphere at radius $R_{\nu}$. Of course, the real situation is more complex and there is no definite radius interior to which neutrinos are in equilibrium at the local thermodynamical conditions and diffuse, and exterior to which they are decoupled from the background and stream freely. The transition between these two limits is continuous and in case of neutrinos, whose reaction rates are strongly energy dependent, it is also a function of the neutrino energy.

The spectral temperature of electron neutrinos will be taken equal to the gas temperature at the assumed neutrinosphere, $k T_{\nu_{\mathrm{e}}}=k T\left(R_{\nu}\right)$. Detailed simulations of 
neutrino transport show that electron antineutrinos have somewhat more energetic spectra. A typical result (e.g., Bruenn 1993; Janka 1991a) is $k T_{\bar{\nu}_{\mathrm{e}}} \approx 1.5 k T_{\nu_{\mathrm{e}}}$, which will be used below. The fact that $\nu_{\mathrm{e}}$ and $\bar{\nu}_{\mathrm{e}}$ spectra are found to be different in detailed models is an indication that the picture drawn above is overly simplified. Nevertheless it is sufficiently accurate for the analysis in this paper. Note that in general the neutrino luminosity $L_{\nu}$ can not be related to the neutrinospheric temperature by the StefanBoltzmann law for blackbody emission of a sphere with radius $R_{\nu}, L_{\nu}=\pi R_{\nu}^{2} \frac{7}{8} a c\left(k T_{\nu}\right)^{4}$. This formula is frequently taken for the combined luminosity of neutrinos plus antineutrinos, assuming their chemical potentials to be zero. However, the effective temperature $k T_{\text {eff }}$, which should be used in the Stefan-Boltzmann law, is typically not equal to the spectral temperature $k T_{\nu}$ (for a discussion, see Janka 1995). Transport simulations show that due to non-equilibrium effects the difference can be quite significant. For this reason two parameters, $T_{\nu}$ and $L_{\nu}$, will be retained here to describe the spectrum and the luminosity of the neutrinos emitted from the neutrinosphere. Moreover, the radius of the neutrinosphere will be considered as the position in the star where the mean value of the cosine of the neutrino propagation angle relative to the radial direction has a value of 0.25 (see Eq. (26) and Janka 1991a,b, 1995).

Keeping in mind the simplifications associated with the concept of the neutrinosphere, the radius $R_{\nu}$ can be defined by the requirement that the effective optical depth to energy exchange for neutrinos with average energy is

$\tau_{\text {eff }}=\int_{R_{\nu}}^{\infty} \mathrm{d} r \kappa_{\text {eff }}(r)=\frac{2}{3}$

(Suzuki 1989). The effective opacity $\kappa_{\text {eff }}$ in case of $\nu_{\mathrm{e}}$ and $\bar{\nu}_{\mathrm{e}}$ is defined from the scattering opacity $\kappa_{\mathrm{sc}}$ and the absorption opacity $\kappa_{\mathrm{a}}$ as

$\kappa_{\mathrm{eff}}=\sqrt{\kappa_{\mathrm{a}}\left(\kappa_{\mathrm{a}}+\kappa_{\mathrm{sc}}\right)}$

(Rybicki \& Lightman 1979; Shapiro \& Teukolsky 1983; Suzuki 1989). Using Eqs. (10) and (11) one obtains for the effective opacity, again averaged over the spectrum of the energy flux which is supposed to have Fermi-Dirac shape with zero degeneracy, in case of electron neutrinos the expression

$$
\begin{aligned}
\kappa_{\mathrm{eff}, \nu_{\mathrm{e}}} & =1.62 \frac{\sigma_{0}\left\langle\epsilon_{\nu_{\mathrm{e}}}^{2}\right\rangle}{\left(m_{\mathrm{e}} c^{2}\right)^{2}} \frac{\rho}{m_{\mathrm{u}}} Y_{\mathrm{n}} \sqrt{1+0.21 \frac{Y_{\mathrm{p}}}{Y_{\mathrm{n}}}} \\
& =2.210^{-7} \rho_{10}\left(\frac{k T_{\nu_{\mathrm{e}}}}{4 \mathrm{MeV}}\right)^{2} Y_{\mathrm{n}} \sqrt{1+0.21 \frac{Y_{\mathrm{p}}}{Y_{\mathrm{n}}}}
\end{aligned}
$$

and in case of electron antineutrinos the analogue result with $Y_{\mathrm{n}} \sqrt{1+0.21 Y_{\mathrm{p}} / Y_{\mathrm{n}}}$ being replaced by $Y_{\mathrm{p}} \sqrt{1+0.21 Y_{\mathrm{n}} / Y_{\mathrm{p}}}$. Since nuclei are nearly completely dissociated into free nucleons and $Y_{\mathrm{n}}$ is larger than $Y_{\mathrm{p}}$, i.e., $Y_{\mathrm{n}} \sim 0.8$ and $Y_{\mathrm{p}} \sim 0.2$, but $k T_{\nu_{\mathrm{e}}}$ is usually somewhat lower than $k T_{\bar{\nu}_{\mathrm{e}}}$, i.e., $k T_{\nu_{\mathrm{e}}} \approx 4 \mathrm{MeV}$ and $k T_{\bar{\nu}_{\mathrm{e}}} \approx 6 \mathrm{MeV}$, we verify the above statement that the effective opacities of $\nu_{\mathrm{e}}$ and $\bar{\nu}_{\mathrm{e}}$ are approximately equal. Assuming equal luminosities, $L_{\nu_{\mathrm{e}}}=L_{\bar{\nu}_{\mathrm{e}}}$, a suitable average value for the effective opacity therefore is

$$
\begin{aligned}
& \left\langle\kappa_{\mathrm{eff}}\right\rangle=\frac{1}{2} \kappa_{\mathrm{eff}, \nu_{\mathrm{e}}}+\frac{1}{2} \kappa_{\mathrm{eff}, \bar{\nu}_{\mathrm{e}}} \\
& \approx 1.510^{-7} \rho_{10}\left(\frac{k T_{\nu_{\mathrm{e}}}}{4 \mathrm{MeV}}\right)^{2}
\end{aligned}
$$

where the composition dependent term in the weighted average has been approximated by $\sqrt{2}$. Knowing the density profile $\rho(r)$, the density $\rho_{\nu}$ at the neutrinosphere can be determined by using Eqs. (16) in (13).

\subsection{The gain radius}

Heating and cooling of the gas outside the neutrinosphere mainly proceed via the charged-current absorption and emission processes of $\nu_{\mathrm{e}}$ and $\bar{\nu}_{\mathrm{e}}$ (Bethe \& Wilson 1985; Bethe 1993, 1995, 1997):

$\nu_{\mathrm{e}}+\mathrm{n} \longleftrightarrow \mathrm{p}+\mathrm{e}^{-}$,

To leading order in the particle energies, the cross sections for neutrino and electron/positron absorption, respectively, are

$\sigma_{\mathrm{a}, \nu_{\mathrm{e}}} \approx \sigma_{\mathrm{a}, \bar{\nu}_{\mathrm{e}}} \approx \frac{3 \alpha^{2}+1}{4} \sigma_{0}\left(\frac{\epsilon_{\nu}}{m_{\mathrm{e}} c^{2}}\right)^{2}$,
$\sigma_{\mathrm{a}, \mathrm{e}^{-}} \approx \sigma_{\mathrm{a}, \mathrm{e}^{+}} \approx \frac{3 \alpha^{2}+1}{8} \sigma_{0}\left(\frac{\epsilon_{\mathrm{e}}}{m_{\mathrm{e}} c^{2}}\right)^{2}$.

At the considered densities and temperatures, fermion phase space blocking and dense-medium effects can be safely ignored, and electrons are relativistic $\left(k T \gtrsim m_{\mathrm{e}} c^{2}\right)$. The heating rate $Q_{\nu_{i}}^{+}$of the stellar medium by neutrinos $\nu_{i}$ is given by

$Q_{\nu_{i}}^{+}=\frac{3 \alpha^{2}+1}{4} \frac{\sigma_{0} c n_{j}}{\left(m_{\mathrm{e}} c^{2}\right)^{2}} \int_{0}^{\infty} \mathrm{d} \epsilon_{\nu} \int_{-1}^{+1} \mathrm{~d} \mu \frac{\mathrm{d}^{2} n_{\nu_{i}}}{\mathrm{~d} \epsilon_{\nu} \mathrm{d} \mu} \epsilon_{\nu}^{3}$,

where $n_{j}$ is the number density of the target nucleons $(j=p, n)$, and $\mathrm{d}^{2} n_{\nu_{i}} /\left(\mathrm{d} \epsilon_{\nu} \mathrm{d} \mu\right)$ the neutrino distribution,

$\frac{\mathrm{d}^{2} n_{\nu_{i}}}{\mathrm{~d} \epsilon_{\nu} \mathrm{d} \mu}=\frac{2 \pi}{(h c)^{3}} f_{\nu_{i}}\left(\epsilon_{\nu}, \mu\right) \epsilon_{\nu}^{2}$

with $f_{\nu_{i}}\left(\epsilon_{\nu}, \mu\right)$ being the neutrino phase space occupation function at some radius $r$, which depends on the neutrino energy $\epsilon_{\nu}$ and the cosine of the angle of neutrino propagation relative to the radial direction, $\mu=\cos \theta$. In Eq. (22) the factor $h$ in the denominator is Planck's constant. Introducing Eq. (22) into Eq. (21) and performing the phase space integration over all energies and angles yields

$Q_{\nu_{i}}^{+}=\frac{3 \alpha^{2}+1}{4} \sigma_{0} n_{j} \frac{\left\langle\epsilon_{\nu_{i}}^{2}\right\rangle}{\left(m_{\mathrm{e}} c^{2}\right)^{2}} \frac{L_{\nu_{i}}}{4 \pi r^{2}\left\langle\mu_{\nu_{i}}\right\rangle}$.

Here the neutrino luminosity $L_{\nu_{i}}$, the average squared neutrino energy $\left\langle\epsilon_{\nu_{i}}^{2}\right\rangle$, and the mean value of the cosine 
of the propagation angle, $\left\langle\mu_{\nu_{i}}\right\rangle$, are calculated from the neutrino phase space occupation function $f_{\nu_{i}}\left(\epsilon_{\nu}, \mu\right)$ by

$$
\begin{aligned}
L_{\nu_{i}} & =4 \pi r^{2} c \frac{2 \pi}{(h c)^{3}} \int_{0}^{\infty} \mathrm{d} \epsilon_{\nu} \int_{-1}^{+1} \mathrm{~d} \mu \mu \epsilon_{\nu}^{3} f_{\nu_{i}}\left(\epsilon_{\nu}, \mu\right) \\
\left\langle\epsilon_{\nu_{i}}^{2}\right\rangle & =\int_{0}^{\infty} \mathrm{d} \epsilon_{\nu} \int_{-1}^{+1} \mathrm{~d} \mu \epsilon_{\nu}^{5} f_{\nu_{i}}\left(\int_{0}^{\infty} \mathrm{d} \epsilon_{\nu} \int_{-1}^{+1} \mathrm{~d} \mu \epsilon_{\nu}^{3} f_{\nu_{i}}\right)^{-1} \\
\left\langle\mu_{\nu_{i}}\right\rangle & =\int_{0}^{\infty} \mathrm{d} \epsilon_{\nu} \int_{-1}^{+1} \mathrm{~d} \mu \mu \epsilon_{\nu}^{3} f_{\nu_{i}}\left(\int_{0}^{\infty} \mathrm{d} \epsilon_{\nu} \int_{-1}^{+1} \mathrm{~d} \mu \epsilon_{\nu}^{3} f_{\nu_{i}}\right)^{-1} .
\end{aligned}
$$

The quantity $\left\langle\mu_{\nu}\right\rangle$ is also called flux factor and can be understood as the ratio of the neutrino energy flux, $L_{\nu} /\left(4 \pi r^{2}\right)$, to the neutrino energy density times $c$. Typically, it is close to 0.25 near the neutrinosphere of $\nu_{\mathrm{e}}$ and $\bar{\nu}_{\mathrm{e}}$ and approaches unity when the neutrino distribution get more and more forward peaked in the limit of free streaming with increasing distance from the neutrinosphere (Janka 1991b, 1992, 1995). The total heating rate $Q_{\nu}^{+}$is the sum of the contributions from $\nu_{\mathrm{e}}$ and $\bar{\nu}_{\mathrm{e}}$ :

$Q_{\nu}^{+}=Q_{\nu_{\mathrm{e}}}^{+}+Q_{\bar{\nu}_{\mathrm{e}}}^{+}$.

To derive a simple expression, one can again assume that $L_{\nu_{\mathrm{e}}} \approx L_{\bar{\nu}_{\mathrm{e}}},\left\langle\epsilon_{\bar{\nu}_{\mathrm{e}}}^{2}\right\rangle \approx 2\left\langle\epsilon_{\nu_{\mathrm{e}}}^{2}\right\rangle$, and that the $\nu_{\mathrm{e}}$ spectrum has Fermi-Dirac shape with zero degeneracy, i.e., $\left\langle\epsilon_{\nu_{\mathrm{e}}}^{2}\right\rangle \approx$ $21\left(k T_{\nu_{\mathrm{e}}}\right)^{2}$. In addition, the equality $\left\langle\mu_{\nu_{\mathrm{e}}}\right\rangle=\left\langle\mu_{\bar{\nu}_{\mathrm{e}}}\right\rangle \equiv\left\langle\mu_{\nu}\right\rangle$ is reasonably well fulfilled because the opacities of electron neutrinos and antineutrinos are very similar and therefore the neutrinospheres of both of them are nearly at the same radius. Putting everything together, the heating rate per unit volume is derived as

$$
\begin{aligned}
Q_{\nu}^{+} & =\frac{3 \alpha^{2}+1}{4} \frac{\sigma_{0}\left\langle\epsilon_{\nu_{\mathrm{e}}}^{2}\right\rangle}{\left(m_{\mathrm{e}} c^{2}\right)^{2}} \frac{\rho}{m_{\mathrm{u}}} \frac{L_{\nu_{\mathrm{e}}}}{4 \pi r^{2}\left\langle\mu_{\nu}\right\rangle}\left(Y_{\mathrm{n}}+2 Y_{\mathrm{p}}\right) \\
& \approx 160 \frac{\rho}{m_{\mathrm{u}}} \frac{L_{\nu_{\mathrm{e}}, 52}}{r_{7}^{2}\left\langle\mu_{\nu}\right\rangle}\left(\frac{k T_{\nu_{\mathrm{e}}}}{4 \mathrm{MeV}}\right)^{2}\left[\frac{\mathrm{MeV}}{\mathrm{s}}\right] .
\end{aligned}
$$

The numerical factor gives the rate in $\mathrm{MeV}$ per baryon, $r_{7}$ is the radius in $10^{7} \mathrm{~cm}$, and $L_{\nu_{\mathrm{e}}, 52}$ the $\nu_{\mathrm{e}}$ luminosity normalized to $10^{52} \mathrm{erg} / \mathrm{s}$. In the layers where most of the heating and cooling between neutrinosphere and shock take place, nuclei are nearly fully dissociated into free nucleons (Bethe 1993, 1995, 1997; Thompson 2000) and $Y_{\mathrm{p}}<Y_{\mathrm{n}}$, therefore using $Y_{\mathrm{n}}+2 Y_{\mathrm{p}} \approx 1$ in the last expression is a reasonable approximation.

The cooling rate of the stellar gas by emission of $\nu_{\mathrm{e}}$ and $\bar{\nu}_{\mathrm{e}}$ is calculated as

$Q_{\nu}^{-}=\frac{3 \alpha^{2}+1}{8} \frac{\sigma_{0} c}{\left(m_{\mathrm{e}} c^{2}\right)^{2}} \int_{0}^{\infty} \mathrm{d} \epsilon \epsilon^{3}\left(n_{\mathrm{p}} \frac{\mathrm{d} n_{\mathrm{e}^{-}}}{\mathrm{d} \epsilon}+n_{n} \frac{\mathrm{d} n_{\mathrm{e}^{+}}}{\mathrm{d} \epsilon}\right)$,

where use was made of Eq. (20), and the distributions of relativistic electrons and positrons are given by

$\frac{\mathrm{d} n_{\mathrm{e}^{ \pm}}}{\mathrm{d} \epsilon}=\frac{8 \pi}{(h c)^{3}} \frac{\epsilon^{2}}{1+\exp \left(\epsilon / k T-\eta_{\mathrm{e}^{ \pm}}\right)}$.
$T(r)$ is the local gas temperature and $\eta_{\mathrm{e}^{ \pm}}$the degeneracy parameter of electrons or positrons, defined as the ratio of the chemical potential to the temperature. A factor of 2 was taken into account as the statistical weight for positive and negative spin states. Inserting Eq. (30) into Eq. (29) one gets for the cooling rate per unit volume

$$
\begin{aligned}
Q_{\nu}^{-}= & \left(3 \alpha^{2}+1\right) \frac{\pi \sigma_{0} c(k T)^{6}}{(h c)^{3}\left(m_{\mathrm{e}} c^{2}\right)^{2}} \frac{\rho}{m_{\mathrm{u}}} \\
& \times\left[Y_{\mathrm{p}} \mathcal{F}_{5}\left(\eta_{\mathrm{e}}\right)+Y_{\mathrm{n}} \mathcal{F}_{5}\left(-\eta_{\mathrm{e}}\right)\right] \\
\approx & 145 \frac{\rho}{m_{\mathrm{u}}}\left(\frac{k T}{2 \mathrm{MeV}}\right)^{6}\left[\frac{\mathrm{MeV}}{\mathrm{s}}\right],
\end{aligned}
$$

where the numerical factor is the rate in $\mathrm{MeV}$ per nucleon when $Y_{\mathrm{n}}+Y_{\mathrm{p}} \approx 1$ and the equilibrium relation $\eta_{\mathrm{e}^{-}}=$ $-\eta_{\mathrm{e}^{+}} \equiv \eta_{\mathrm{e}}$ with $\eta_{\mathrm{e}} \approx 0$ are used. The latter approximation is good in the shock-heated layers because the electron fraction $Y_{\mathrm{e}}=n_{\mathrm{e}} / n_{\mathrm{b}}$ and thus the electron degeneracy is rather low and $\mathrm{e}^{ \pm}$pairs are abundant. $\mathcal{F}_{5}(\eta)$ is the Fermi integral for relativistic particles,

$\mathcal{F}_{j}(\eta)=\int_{0}^{\infty} \mathrm{d} x \frac{x^{j}}{1+\exp (x-\eta)}$

with $\mathcal{F}_{5}(0) \approx 118$. (Useful formulae for sums and differences of these Fermi integrals can be found in Bludman \& Van Riper 1978, and simple approximations in Takahashi et al. 1978.)

Heating balances cooling at the gain radius, i.e., the gain radius $R_{\mathrm{g}}$ has to fulfill the condition $Q_{\nu}^{+}=Q_{\nu}^{-}$by definition. With Eqs. (28) and (31) one obtains the following relation:

$R_{\mathrm{g}, 7}\left(\frac{k T_{\mathrm{g}}}{2 \mathrm{MeV}}\right)^{3} \approx 1.05 \sqrt{\frac{L_{\nu_{\mathrm{e}}, 52}}{\left\langle\mu_{\nu}\right\rangle_{\mathrm{g}}}}\left(\frac{k T_{\nu_{\mathrm{e}}}}{4 \mathrm{MeV}}\right)$

$R_{\mathrm{g}, 7}$ is the gain radius in units of $10^{7} \mathrm{~cm}$ and $T_{\mathrm{g}}=T\left(R_{\mathrm{g}}\right)$ the temperature at the gain radius. Depending on the position of the gain radius, $\left\langle\mu_{\nu}\right\rangle_{\mathrm{g}}$ is a factor somewhere between 0.25 (value at the neutrinosphere) and unity (limit for $r \rightarrow \infty)$.

\subsection{The EoS transition radius}

It is interesting to consider the conditions for which the pressure is dominated by nonrelativistic nucleons or radiation plus relativistic $\mathrm{e}^{ \pm}$pairs $(k T \gtrsim 0.5 \mathrm{MeV})$. In the first case $P \approx P_{\mathrm{b}}=k T \rho / m_{\mathrm{u}}$, if nuclei are fully dissociated into free nucleons. In the latter case $P \approx$ $P_{\mathrm{r}}=P_{\mathrm{e}^{ \pm}}+P_{\gamma} \approx \frac{11}{12} a_{\gamma}(k T)^{4}$, when $\eta_{\mathrm{e}} \approx 0$ is again assumed for the electron degeneracy and the constant is $a_{\gamma}=8 \pi^{5} /\left[15(h c)^{3}\right] \approx 8.5610^{31} \mathrm{MeV}^{-3} \mathrm{~cm}^{-3}$. Setting $P_{\mathrm{b}}$ equal to $P_{\mathrm{r}}$ gives

$\frac{(k T)^{3}}{\rho}=\frac{12}{11} \frac{1}{m_{\mathrm{u}} a_{\gamma}}$

or, using the temperature $k T \approx k T_{\nu_{\mathrm{e}}} \approx 4 \mathrm{MeV}$ (compare Fig. 2),

$\left(\frac{k T}{4 \mathrm{MeV}}\right)^{3} \rho_{10}^{-1} \cong 1.2$. 
This means that the transition from the baryondominated to the radiation-dominated regime occurs at a density significantly below that of the neutrinosphere. The latter is typically above $10^{11} \mathrm{~g} / \mathrm{cm}^{3}$. When the electron degeneracy is negligibly small, the contributions of relativistic and nonrelativistic gas components to the pressure are equal for a value of the radiation entropy per nucleon of $s_{\mathrm{r}}=s_{\mathrm{e}^{ \pm}}+s_{\gamma} \approx\left(\varepsilon_{\mathrm{r}}+P_{\mathrm{r}}\right) /\left(k T \rho / m_{\mathrm{u}}\right)=4 P_{\mathrm{r}} / P_{\mathrm{b}}=4$. Since the energy density of relativistic particles is $\varepsilon_{\mathrm{r}}=3 P_{\mathrm{r}}$, whereas $\varepsilon_{\mathrm{b}}=3 P_{\mathrm{b}} / 2$ for nonrelativistic particles, the relativistic electrons-positron pairs and photons dominate the energy density at such conditions. The main contribution to the entropy, however, then still comes from nucleons and nuclei (cf. Fig. 8 in Woosley et al. 1986).

\subsection{The shock radius and infall region}

Conservation of the mass flow, momentum flow and energy flow across the discontinuity of the shock front is expressed by the three Rankine-Hugoniot conditions

$\rho_{\mathrm{p}} u_{\mathrm{p}}=\rho_{\mathrm{s}} u_{\mathrm{s}}$

$P_{\mathrm{p}}+\rho_{\mathrm{p}} u_{\mathrm{p}}^{2}=P_{\mathrm{s}}+\rho_{\mathrm{s}} u_{\mathrm{s}}^{2}$,

$\frac{1}{2} u_{\mathrm{p}}^{2}+w_{\mathrm{p}}-q_{\mathrm{d}}=\frac{1}{2} u_{\mathrm{s}}^{2}+w_{\mathrm{s}}$

where the indices $\mathrm{p}$ and $\mathrm{s}$ denote quantities just ahead and behind the shock, respectively (see Fig. 2), $w=(\varepsilon+$ $P) / \rho$ is the enthalpy per unit mass, $q_{\mathrm{d}}$ the nuclear binding energy per unit mass absorbed by photodisintegration of nuclei within the shock front, and $u=v-U_{\mathrm{s}}$ the fluid velocity relative to the shock when $U_{\mathrm{s}}=\dot{R}_{\mathrm{s}}$ is the shock velocity and $v$ the gas velocity relative to the center of the star. Note that in the infall region $v$ has negative sign.

With the definition $\beta \equiv \rho_{\mathrm{s}} / \rho_{\mathrm{p}}$, Eq. (36) gives $u_{\mathrm{s}}=$ $u_{\mathrm{p}} / \beta$, which can be used to eliminate $u_{\mathrm{s}}$ from Eq. (37). For a strong shock, i.e., $P_{\mathrm{s}} \gg P_{\mathrm{p}}$, this yields

$P_{\mathrm{s}} \approx\left(1-\frac{1}{\beta}\right) \rho_{\mathrm{p}}\left(v_{\mathrm{p}}-U_{\mathrm{s}}\right)^{2}$

Combining Eqs. (36)-(38) one further finds

$w_{\mathrm{s}}-w_{\mathrm{p}}=\frac{1}{2}\left(P_{\mathrm{s}}-P_{\mathrm{p}}\right)\left(\frac{1}{\rho_{\mathrm{s}}}+\frac{1}{\rho_{\mathrm{p}}}\right)-q_{\mathrm{d}}$.

With $P_{\mathrm{p}} \ll P_{\mathrm{s}}, w_{\mathrm{p}} \ll w_{\mathrm{s}}$ and $w_{\mathrm{s}} \approx 4 P_{\mathrm{s}} / \rho_{\mathrm{s}}$ for the radiation-dominated gas in the postshock region, Eq. (40) can be rewritten as

$$
\frac{\rho_{\mathrm{s}}}{\rho_{\mathrm{p}}} \approx 7+\frac{2 q_{\mathrm{d}} \rho_{\mathrm{s}}}{P_{\mathrm{s}}} \approx \frac{7}{4-3 \sqrt{1+14 q_{\mathrm{d}} /\left(9 u_{\mathrm{p}}^{2}\right)}}
$$

where in the second transformation Eq. (39) was used to replace $P_{\mathrm{s}} / \rho_{\mathrm{s}}$. This shows that for a relativistic gas the density jump in a strong shock is a factor of 7 . Energy consumed by photodissociation of nuclei increases the density contrast between preshock and postshock region (Thompson 2000). In a more general treatment, retaining $w_{\mathrm{p}}$ and taking into account the (subdominant) contributions from nonrelativistic nucleons to the gas pressure behind the shock (but still using $P_{\mathrm{p}} \ll \rho_{\mathrm{p}} u_{\mathrm{p}}^{2}$ in the infall region) one also derives the right hand side of Eq. (41), now with the expression

$q^{*} \equiv q_{\mathrm{d}}-w_{\mathrm{p}}-\frac{3}{2 m_{\mathrm{u}}} \sum_{i} Y_{i} k T_{\mathrm{s}}$

instead of $q_{\mathrm{d}}$ in the denominator. This means that the density discontinuity is also affected by the preshock enthalpy and the thermal pressure of nucleons and nuclei behind the shock (the nuclear composition is accounted for by the sum of the number fractions, $\left.\sum_{i} Y_{i}\right)$. Considering $q_{\mathrm{d}}$ to be several $\mathrm{MeV} / m_{\mathrm{u}}, k T_{\mathrm{s}} \sim 1 \mathrm{MeV}$, and the preshock medium to be dominated by relativistic, degenerate electrons in which case $w_{\mathrm{p}} \approx \zeta_{\mathrm{e}} Y_{\mathrm{e}} / m_{\mathrm{u}}$ with an electron chemical potential $\zeta_{\mathrm{e}}=\eta_{\mathrm{e}} k T$ of a few $\mathrm{MeV}$, one can see that all terms in Eq. (42) are of the same order and therefore equally important.

The preshock region is not affected by the postshock conditions. Because the shock moves supersonically relative to the medium ahead of it, sound waves cannot transport information in this direction. The matter there falls into the shock with a significant fraction of the free-fall velocity,

$v_{\mathrm{p}}=-\alpha \sqrt{\frac{2 G \widetilde{M}}{R_{\mathrm{s}}}}$

with $\alpha \sim 1 / \sqrt{2}$ (Bethe 1990, 1993; Bruenn 1993). Ahead of the shock free nucleons are absent and therefore $\nu_{\mathrm{e}}$ and $\bar{\nu}_{\mathrm{e}}$ absorption does not play a role, but neutrinos interact with nuclei by coherent scatterings. The opacity of the latter reaction scales roughly with $N^{2} / A$ when $N$ is the neutron number and $A$ the mass number of the nuclei, and the total neutrino opacity of the preshock medium turns out to be close to the result of Eq. (12). Therefore the momentum transfer by neutrinos was again taken into account by using $\widetilde{M}$ instead of $M$ in Eq. (43). Plugging Eq. (43) into the rate at which mass falls into the shock, $\dot{M}=4 \pi R_{\mathrm{s}}^{2} \rho_{\mathrm{p}} v_{\mathrm{p}}(<0)$, gives the density just above the shock:

$\rho_{\mathrm{p}}=-\frac{\dot{M}}{4 \pi \alpha \sqrt{2 G \widetilde{M}} R_{\mathrm{s}}^{3 / 2}}$.

On the other hand, if the original presupernova material has a density distribution $\rho_{0}\left(r_{0}\right)=H r_{0}^{-3}$ with $H$ being a constant, then mass conservation yields a density at the footpoint of the shock at time $t$ after the start of the collapse of

$\rho_{\mathrm{p}}=\frac{2}{3} \frac{H}{\alpha \sqrt{2 G \widetilde{M}}} t^{-1} R_{\mathrm{s}}^{-3 / 2}$

(Bethe 1990, 1993; see also Cooperstein et al. 1984). Comparing Eqs. (44) and (45) one finds that the rate at which mass crosses the shock in this case is

$\dot{M}=-\frac{8 \pi}{3} \frac{H}{t}$ 
which depends on the structure of the progenitor star through the constant $H$ and decreases with time.

\section{Structure of the atmosphere}

Within the supernova shock, the infalling matter is strongly decelerated to a velocity $v_{\mathrm{s}}=\left(v_{\mathrm{p}}-U_{\mathrm{s}}\right) / \beta+U_{\mathrm{s}}$. For a stalled shock, $\left|v_{\mathrm{s}}\right| \ll\left|v_{\mathrm{p}}\right|$. Compared to the internal energy and the gravitational energy, the kinetic energy behind the shock is therefore negligibly small. The gas is further slowed down as it moves inward and settles onto the nascent neutron star. Between neutrinosphere and shock front $\mathrm{d} v / \mathrm{d} t \approx 0$ is therefore a good assumption, i.e., the stellar structure is well approximated by hydrostatic equilibrium (Chevalier 1989; Bethe 1993, 1995; Fryer et al. 1996). Combining Eqs. (2) and (3) and using Eq. (6), the equation of hydrostatic equilibrium is found to be

$$
-\frac{1}{\rho} \frac{\partial P}{\partial r}-\frac{G \widetilde{M}}{r^{2}}=0 \text {. }
$$

In the following, the solutions of this equation in the layers between neutrinosphere $R_{\nu}$ and EoS transition radius $R_{\text {eos }}$ and between $R_{\text {eos }}$ and shock position $R_{\mathrm{S}}$ will be derived.

\subsection{Hydrostatic equilibrium between $R_{\nu}$ and $R_{\text {eos }}$}

When nonrelativistic baryons dominate the pressure and relativistic electrons contribute, but positrons and radiation can be ignored because the electrons are mildly degenerate, the pressure can be expressed as

$$
\begin{aligned}
P \approx P_{\mathrm{b}}+P_{\mathrm{e}^{-}} & =\frac{\rho}{m_{\mathrm{u}}} k T\left(1+\frac{Y_{\mathrm{e}}}{3} \frac{\mathcal{F}_{3}\left(\eta_{\mathrm{e}}\right)}{\mathcal{F}_{2}\left(\eta_{\mathrm{e}}\right)}\right) \\
& \equiv f_{\mathrm{g}} \frac{\rho}{m_{\mathrm{u}}} k T .
\end{aligned}
$$

Since $Y_{\mathrm{e}}$ and the electron degeneracy do not vary strongly, the factor $f_{\mathrm{g}}$ can be considered as constant. Between $R_{\nu}$ and $R_{\text {eos }}$ also the temperature is a slowly changing quantity, $T(r) \approx T_{\nu} \approx T_{\nu_{\mathrm{e}}}$ (compare Fig. 2). Hydrostatic equilibrium therefore implies

$\rho(r)=\rho_{\nu} \exp \left[-\frac{G \widetilde{M} m_{\mathrm{u}}}{f_{\mathrm{g}} k T_{\nu} R_{\nu}}\left(1-\frac{R_{\nu}}{r}\right)\right]$,

where $\rho_{\nu}$ is the density at the neutrinosphere. Near the neutrinosphere, $r \approx R_{\nu}$, this can be approximated by

$$
\rho(r) \approx \rho_{\nu} \exp \left(-\frac{x}{h}\right)
$$

with $\quad x \equiv r-R_{\nu} \quad$ and $\quad h \equiv f_{\mathrm{g}} \frac{k T_{\nu} R_{\nu}^{2}}{G \widetilde{M} m_{\mathrm{u}}}$.

Using typical numbers gives

$h \approx 2.910^{4} f_{\mathrm{g}} R_{\nu, 6}^{2}\left(\frac{k T_{\nu}}{4 \mathrm{MeV}}\right)\left(\frac{\widetilde{M}}{M}\right)^{-1}[\mathrm{~cm}]$,

where $R_{\nu, 6}$ is the radius of the neutrinosphere in units of $10^{6} \mathrm{~cm}$.
The density declines exponentially outside the neutrinosphere with a scale height $h \ll r$, forming a sharp "cliff" (Bethe \& Wilson 1985; Bethe 1990; Woosley 1993a). For this reason the effective optical depth is dominated by the immediate vicinity of the neutrinosphere. Therefore the integration in Eq. (13) can be performed, using Eq. (50) for the density in the effective opacity of Eq. (16), to derive the neutrinospheric density (normalized to $10^{10} \mathrm{~g} / \mathrm{cm}^{3}$ ) as

$\rho_{\nu, 10} \approx 150 f_{\mathrm{g}}^{-1} R_{\nu, 6}^{-2}\left(\frac{\widetilde{M}}{M_{\odot}}\right)\left(\frac{k T_{\nu}}{4 \mathrm{MeV}}\right)^{-3}$.

This result confirms that the density of the transition from the baryon-dominated to the radiation-dominated regime (Eq. (35)) is significantly lower than $\rho_{\nu}$.

\subsection{Hydrostatic equilibrium between $R_{\text {eos }}$ and $R_{s}$}

In the radiation-dominated region a large part of the pressure is due to relativistic electron-positron pairs and photons, but also contributions from nucleons and nuclei with number fractions $Y_{i}$ might not be negligible, therefore

$$
\begin{aligned}
P & =P_{\gamma}+P_{\mathrm{e}^{ \pm}}+P_{\mathrm{b}} \\
& =\frac{a_{\gamma}}{3}(k T)^{4}\left[\frac{11}{4}+\frac{15}{2 \pi^{4}} \eta_{\mathrm{e}}^{2}\left(\pi^{2}+\frac{\eta_{\mathrm{e}}^{2}}{2}\right)+\frac{3 \rho k T \sum_{i} Y_{i}}{m_{\mathrm{u}} a_{\gamma}(k T)^{4}}\right] \\
& \equiv P_{\mathrm{r}}\left(1+\frac{4}{g_{\mathrm{r}} s_{\gamma}}\right) \equiv f_{\mathrm{r}} P_{\mathrm{r}},
\end{aligned}
$$

where $P_{\mathrm{r}}$ is the pressure associated with relativistic particles,

$P_{\mathrm{r}}=g_{\mathrm{r}} P_{\gamma}=\frac{1}{3} a_{\gamma} g_{\mathrm{r}}(k T)^{4}$,

with $\quad g_{\mathrm{r}} \equiv \frac{11}{4}+\frac{15}{2 \pi^{4}} \eta_{\mathrm{e}}^{2}\left(\pi^{2}+\frac{1}{2} \eta_{\mathrm{e}}^{2}\right)$,

$s_{\gamma}$ is the entropy per nucleon carried by photons, $s_{\gamma}=$ $4 a_{\gamma}(k T)^{3} /\left(3 \rho / m_{\mathrm{u}}\right)$, and $\sum_{i} Y_{i} \approx 1$ because of the nearly complete disintegration of nuclei. If both the factor $g_{\mathrm{r}}$ and $s_{\gamma}$ are constant (which is roughly fulfilled in the radiationdominated region between $R_{\text {eos }}$ and $R_{\mathrm{s}}$ where the electron degeneracy parameter $\eta_{\mathrm{e}}$ divided by $\pi$ is small, and, as was discussed in Sect. 2, convective processes tend to homogenize the total entropy and thus also the radiation entropy; see Bethe 1996b) then also $f_{\mathrm{r}}$ can be considered as constant. In this case the pressure is simply proportional to $(k T)^{4}$, both for the contribution from nucleons and for the contribution from photons plus electron-positron pairs (for a detailed discussion, see Bethe 1993).

This implies that the density $\rho$ is proportional to $T^{3}$, i.e.,

$P=f_{\mathrm{r}} g_{\mathrm{r}} \frac{a_{\gamma}}{3}(k T)^{4}=K \rho^{4 / 3}$.

Note that Eq. (56) is valid more generally than for radiation-dominated conditions (Bethe 1996b). Using

$n_{\mathrm{e}}=Y_{\mathrm{e}} \frac{\rho}{m_{\mathrm{u}}}=\frac{8 \pi}{3} \frac{(k T)^{3}}{(h c)^{3}} \eta_{\mathrm{e}}\left(\pi^{2}+\eta_{\mathrm{e}}^{2}\right)$, 
the coefficient $K$ can be determined as

$$
K=f_{\mathrm{r}} g_{\mathrm{r}} \frac{a_{\gamma}}{3}(h c)^{4}\left(\frac{3}{8 \pi} \frac{Y_{\mathrm{e}}}{m_{\mathrm{u}} \eta_{\mathrm{e}}\left(\pi^{2}+\eta_{\mathrm{e}}^{2}\right)}\right)^{4 / 3} .
$$

If $K$ in Eq. (58) is approximately constant, which is typically fulfilled in the region between $R_{\mathrm{g}}$ and $R_{\mathrm{S}}$ (Bethe $1996 \mathrm{~b})$, Eq. (56) is a useful representation of the equation of state.

With Eq. (47), one can now determine the density distribution between $R_{\text {eos }}$ and $R_{\mathrm{s}}$ in hydrostatic equilibrium as

$$
\rho(r)=\left[\rho_{\mathrm{s}}^{1 / 3}+\frac{1}{4} \frac{G \widetilde{M}}{K}\left(\frac{1}{r}-\frac{1}{R_{\mathrm{s}}}\right)\right]^{3} .
$$

Inserting this in Eq. (56) and setting $K=P_{\mathrm{s}} / \rho_{\mathrm{s}}^{4 / 3}$, the pressure as a function of radius is obtained,

$P(r)=\left[P_{\mathrm{s}}^{1 / 4}+\frac{1}{4} \frac{G \widetilde{M}}{K^{3 / 4}}\left(\frac{1}{r}-\frac{1}{R_{\mathrm{s}}}\right)\right]^{4}$,

and $k T(r)$ can also be found from Eq. (56) as

$$
k T(r)=k T_{\mathrm{s}}+\frac{1}{4}\left(\frac{3}{f_{\mathrm{r}} g_{\mathrm{r}} a_{\gamma}}\right)^{1 / 4} \frac{G \widetilde{M}}{K^{3 / 4}}\left(\frac{1}{r}-\frac{1}{R_{\mathrm{s}}}\right)
$$

when $k T_{\mathrm{s}}=\left[3 P_{\mathrm{s}} /\left(f_{\mathrm{r}} g_{\mathrm{r}} a_{\gamma}\right)\right]^{1 / 4}$ is used. If the densitypressure relation is more general than Eq. (56), namely $P=K \rho^{\gamma}$ with $K$ being constant, hydrostatic equilibrium implies

$\rho(r)=\left[\rho_{\mathrm{s}}^{\gamma-1}+\frac{\gamma-1}{\gamma} \frac{G \widetilde{M}}{K}\left(\frac{1}{r}-\frac{1}{R_{\mathrm{s}}}\right)\right]^{1 /(\gamma-1)}$,

which replaces Eq. (59).

Instead of the general solutions, Eqs. (59)-(61), simple power-laws,

$$
\begin{aligned}
& \rho(r)=\rho_{\mathrm{s}}\left(\frac{R_{\mathrm{s}}}{r}\right)^{3}, k T(r)=k T_{\mathrm{s}} \frac{R_{\mathrm{S}}}{r}, \\
& P(r)=P_{\mathrm{s}}\left(\frac{R_{\mathrm{s}}}{r}\right)^{4},
\end{aligned}
$$

yield a good approximation for the hydrostatic atmosphere, if $K$ fulfills the condition $K=G \widetilde{M} /\left(4 R_{\mathrm{s}} \rho_{\mathrm{s}}^{1 / 3}\right)$. Since $K=P_{\mathrm{s}} / \rho_{\mathrm{s}}^{4 / 3}$, this is equivalent to the requirement that

$$
\frac{P_{\mathrm{s}}}{\rho_{\mathrm{s}}}=\frac{G \widetilde{M}}{4 R_{\mathrm{s}}}
$$

On the other hand, from Eqs. (39) and (43) one gets

$$
\begin{aligned}
\frac{P_{\mathrm{s}}}{\rho_{\mathrm{s}}} & \approx \frac{\beta-1}{\beta^{2}} \frac{2 \alpha^{2} G \widetilde{M}}{R_{\mathrm{s}}}\left(1-\frac{U_{\mathrm{s}}}{v_{\mathrm{p}}}\right)^{2} \\
& \sim \frac{6}{49} \frac{G \widetilde{M}}{R_{\mathrm{s}}}\left(1-\frac{U_{\mathrm{s}}}{v_{\mathrm{p}}}\right)^{2} .
\end{aligned}
$$

The numerical factor on the right hand side of Eq. (65) was obtained with $\alpha \sim 1 / \sqrt{2}$ (Bethe 1990, 1993) and $\beta \sim 7$ (Eq. (41)). Equation (65) shows that the requirement of Eq. (64) is reasonably well, although for small shock radii not very well, fulfilled. In the following the power-laws of Eq. (63) will therefore only serve to facilitate analytical evaluation, and the use of this approximate solution of hydrostatic equilibrium will be avoided where inconsistencies might result.

With Eqs. (33) and (63) the gain radius $R_{\mathrm{g}}$ and the conditions at the gain radius can be expressed in terms of the properties at the shock front and the characteristic parameters $\left(T_{\nu_{\mathrm{e}}}, L_{\nu_{\mathrm{e}}}\right)$ of the neutrino emission. Inserting the relation $k T_{\mathrm{g}}=k T_{\mathrm{s}}\left(R_{\mathrm{s}} / R_{\mathrm{g}}\right)$ into Eq. (33) yields the gain radius (in units of $10^{7} \mathrm{~cm}$ ),

$R_{\mathrm{g}, 7} \approx 0.98 R_{\mathrm{s}, 7}^{\frac{3}{2}}\left(k T_{\mathrm{s}, 2}\right)^{\frac{3}{2}}\left(k T_{\nu_{\mathrm{e}}, 4}\right)^{-\frac{1}{2}}\left(\frac{L_{\nu_{\mathrm{e}}, 52}}{\left\langle\mu_{\nu}\right\rangle_{\mathrm{g}}}\right)^{-\frac{1}{4}}$

and for the temperature at the gain radius one gets

$k T_{\mathrm{g}, 2} \approx 1.02 R_{\mathrm{s}, 7}^{-\frac{1}{2}}\left(k T_{\mathrm{s}, 2}\right)^{-\frac{1}{2}}\left(k T_{\nu_{\mathrm{e}}, 4}\right)^{\frac{1}{2}}\left(\frac{L_{\nu_{\mathrm{e}}, 52}}{\left\langle\mu_{\nu}\right\rangle_{\mathrm{g}}}\right)^{\frac{1}{4}}$

where $k T_{\mathrm{g}, 2}=k T_{\mathrm{g}} /(2 \mathrm{MeV})$ and $k T_{\nu_{\mathrm{e}}, 4}$ is the neutrinospheric temperature and $k T_{\mathrm{s}, 2}$ the postshock temperature normalized to $4 \mathrm{MeV}$ and $2 \mathrm{MeV}$, respectively.

The assumptions made in this section to solve the equation of hydrostatic equilibrium in the layer between $R_{\text {eos }}$ and $R_{\mathrm{s}}$ do not seem to be very restrictive, because two-dimensional as well as one-dimensional simulations without convection (e.g., Bruenn 1993; Janka \& Müller 1996, Fig. 6; Rampp 2000) yield density and temperature profiles in the postshock region which are very close to power laws with power law indices around 3 and 1, respectively. Near $R_{\text {eos }}$ the contributions of relativistic and nonrelativistic gas components will become equally important. Here the exponentially steep density decline just outside the neutrinosphere must change to the power-law behavior behind the shock, and both of these limiting solutions will not provide a good description. The exact structure in the intermediate layer between $R_{\text {eos }}$ and $R_{\mathrm{g}}$, however, does not play an important role in the further discussion and therefore a more accurate treatment is not necessary.

\section{Heating and cooling}

To discuss energy deposition and emission of neutrinos exterior to the neutrinosphere, one starts with the energy equation for $\nu_{\mathrm{e}}$ plus $\bar{\nu}_{\mathrm{e}}$, which is

$\frac{1}{4 \pi r^{2}} \frac{\partial L_{\nu}}{\partial r}=-Q_{\nu}^{+}+Q_{\nu}^{-}$

where $L_{\nu}=L_{\nu_{\mathrm{e}}}+L_{\bar{\nu}_{\mathrm{e}}}$ and $Q_{\nu}^{+}$and $Q_{\nu}^{-}$are the heating and cooling rates of the stellar medium as given by Eqs. (28) and (31), respectively. In writing Eq. (68), stationarity was assumed for the neutrinos, which is justified because the neutrino emission of the accreting neutrino star changes 
on a timescale which is typically longer than other relevant timescales of the discussed problem. From Eq. (68) the net effect of heating or cooling in a layer between radii $r_{1}$ and $r_{2}$ can be deduced as

$\int_{r_{1}}^{r_{2}} \mathrm{~d} r 4 \pi r^{2}\left(Q_{\nu}^{+}-Q_{\nu}^{-}\right)=L_{\nu}\left(r_{1}\right)-L_{\nu}\left(r_{2}\right)$.

Refering to Eqs. (23) and (27), a suitable spectral and flavor average for the absorption coefficient of $\nu_{\mathrm{e}}$ and $\bar{\nu}_{\mathrm{e}}$ can be defined as

$\left\langle\kappa_{\mathrm{a}}\right\rangle=\frac{Q_{\nu}^{+}}{L_{\nu} /\left(4 \pi r^{2}\left\langle\mu_{\nu}\right\rangle\right)}$,

when $\left\langle\mu_{\nu_{\mathrm{e}}}\right\rangle \approx\left\langle\mu_{\bar{\nu}_{\mathrm{e}}}\right\rangle \equiv\left\langle\mu_{\nu}\right\rangle$ is used. Plugging this into Eq. (68) gives

$\frac{\partial L_{\nu}}{\partial r}=-\left\langle\kappa_{\mathrm{a}}\right\rangle \frac{L_{\nu}}{\left\langle\mu_{\nu}\right\rangle}+4 \pi r^{2} Q_{\nu}^{-}$.

The neutrino luminosity as a function of radius $r \geq r_{0}$ is the general solution of Eq. (71):

$$
\begin{aligned}
L_{\nu}(r) & =\exp \left\{-\int_{r_{0}}^{r} \mathrm{~d} r^{\prime} \frac{\left\langle\kappa_{\mathrm{a}}\right\rangle}{\left\langle\mu_{\nu}\right\rangle}\right\} L_{\nu}\left(r_{0}\right) \\
& +\int_{r_{0}}^{r} \mathrm{~d} r^{\prime} 4 \pi\left(r^{\prime}\right)^{2} Q_{\nu}^{-} \exp \left\{-\int_{r^{\prime}}^{r} \mathrm{~d} r^{\prime \prime} \frac{\left\langle\kappa_{\mathrm{a}}\right\rangle}{\left\langle\mu_{\nu}\right\rangle}\right\} .
\end{aligned}
$$

The first exponential factor represents the absorption damping of the luminosity in the shell between $r_{0}$ and $r$, the second exponential factor the reabsorption of neutrinos emitted at $r^{\prime}$ in the layer enclosed by radii $r^{\prime}$ and $r$.

\subsection{Heating and cooling between $R_{\nu}$ and $R_{g}$}

Here the lower boundary of the considered volume is the neutrinosphere at radius $r_{0}=R_{\nu}$. Since both $\left\langle\kappa_{\mathrm{a}}\right\rangle \propto \rho(r)$ (cf. Eqs. (70) and (28)) and $Q_{\nu}^{-} \propto \rho(r)$ (cf. Eq. (31)) are steep functions of the radius in the region between $R_{\nu}$ and $R_{\mathrm{g}}$, where the density drops exponentially, most of the absorption and emission occurs in the immediate vicinity of the neutrinosphere. Therefore the neutrino luminosity at the gain radius, $L_{\nu}\left(R_{\mathrm{g}}\right)$, can be approximated by the limit for $r \rightarrow \infty$ of Eq. (72), and the integral $\int_{r^{\prime}}^{r} \mathrm{~d} r^{\prime \prime}\left\langle\kappa_{\mathrm{a}}\right\rangle /\left\langle\mu_{\nu}\right\rangle$ can be replaced by $\int_{R_{\nu}}^{\infty} \mathrm{d} r\left\langle\kappa_{\mathrm{a}}\right\rangle /\left\langle\mu_{\nu}\right\rangle$. This leads to

$$
\begin{aligned}
L_{\nu}\left(R_{\mathrm{g}}\right) \approx \exp & \left\{-\int_{R_{\nu}}^{\infty} \mathrm{d} r \frac{\left\langle\kappa_{\mathrm{a}}\right\rangle}{\left\langle\mu_{\nu}\right\rangle}\right\} \\
\times & {\left[L_{\nu}\left(R_{\nu}\right)+\int_{R_{\nu}}^{\infty} \mathrm{d} r 4 \pi r^{2} Q_{\nu}^{-}\right] . }
\end{aligned}
$$

To evaluate the exponential damping factor, $\left\langle\kappa_{\mathrm{a}}\right\rangle$ is expressed by Eq. (70), making use of Eq. (28) and $L_{\nu}=$ $2 L_{\nu_{\mathrm{e}}}$. The neutrino spectrum is assumed not to change outside the neutrinosphere. With Eq. (50) the integral over the density profile becomes $\int_{R_{\nu}}^{\infty} \mathrm{d} r \rho(r) \approx \rho_{\nu} h$. Employing Eqs. (51) and (52), one finds

$\int_{R_{\nu}}^{\infty} \mathrm{d} r \frac{\left\langle\kappa_{\mathrm{a}}\right\rangle}{\left\langle\mu_{\nu}\right\rangle} \approx \frac{0.42}{\widetilde{\left\langle\mu_{\nu}\right\rangle}} \equiv a$

where $\widetilde{\left\langle\mu_{\nu}\right\rangle}$ denotes a radial average of the flux factor $\left\langle\mu_{\nu}\right\rangle$ in the layer between $R_{\nu}$ and $R_{\mathrm{g}}$. The energy loss integral is calculated with Eq. (31) where $T(r) \approx T_{\nu_{\mathrm{e}}}$ is used near the neutrinosphere. With $\int_{R_{\nu}}^{\infty} \mathrm{d} r r^{2} \rho(r) \approx$ $\rho_{\nu} h\left[h^{2}+\left(R_{\nu}+h\right)^{2}\right] \approx \rho_{\nu} R_{\nu}^{2} h$ (because $h \ll R_{\nu}$, cf. Eq. (51)) and Eqs. (51) and (52) this leads to:

$\int_{R_{\nu}}^{\infty} \mathrm{d} r 4 \pi r^{2} Q_{\nu}^{-} \approx 4.910^{51} R_{\nu, 6}^{2}\left(\frac{k T_{\nu_{\mathrm{e}}}}{4 \mathrm{MeV}}\right)^{4}\left[\frac{\mathrm{erg}}{\mathrm{s}}\right] \equiv b$.

Equation (73) now becomes

$L_{\nu}\left(R_{\mathrm{g}}\right) \approx \mathrm{e}^{-a}\left[L_{\nu}\left(R_{\nu}\right)+b\right]$,

and the total energy exchange between $R_{\nu}$ and $R_{\mathrm{g}}$ according to Eq. (69) therefore is

$L_{\nu}\left(R_{\mathrm{g}}\right)-L_{\nu}\left(R_{\nu}\right) \equiv L_{\mathrm{acc}} \approx\left(\mathrm{e}^{-a}-1\right) L_{\nu}\left(R_{\nu}\right)+\mathrm{e}^{-a} b$.

Since $R_{\mathrm{g}}$ separates the layer of neutrino cooling from the one of neutrino heating, the region between $R_{\nu}$ and $R_{\mathrm{g}}$ must lose energy by neutrino emission. Therefore the neutrino luminosity at $R_{\mathrm{g}}$ must be larger than $L_{\nu}\left(R_{\nu}\right)$, and $L_{\text {acc }}$ represents the luminosity associated with the accretion of matter through the gain radius onto the surface of the nascent neutron star. The requirement $L_{\mathrm{acc}} \geq 0$ constrains the luminosity of the neutron star core relative to the product $R_{\nu}^{2}\left(k T_{\nu_{\mathrm{e}}}\right)^{4}$ by the inequality

$L_{\nu}\left(R_{\nu}\right) \leq b\left(\mathrm{e}^{a}-1\right)^{-1}$.

Provided the core luminosity can be expressed in terms of blackbody emission of temperature $T_{\nu_{\mathrm{e}}}$,

$$
\begin{aligned}
L_{\nu}\left(R_{\nu}\right) & \approx 4 \pi R_{\nu}^{2} \frac{c}{4} \frac{7}{8} a_{\gamma}\left(k T_{\nu_{\mathrm{e}}}\right)^{4} \\
& \approx 2.910^{51} R_{\nu, 6}^{2}\left(\frac{k T_{\nu_{\mathrm{e}}}}{4 \mathrm{MeV}}\right)^{4}\left[\frac{\mathrm{erg}}{\mathrm{s}}\right],
\end{aligned}
$$

the consistency condition translates into the relation

$\widetilde{\left\langle\mu_{\nu}\right\rangle} \gtrsim 0.42$

which is satisfied above the neutrinosphere in the layer between $R_{\nu}$ and $R_{\mathrm{g}}$.

\subsection{Heating and cooling between $R_{g}$ and $R_{s}$}

For reasons of simplicity it will be assumed that in the layer bounded by $R_{\mathrm{g}}$ and $R_{\mathrm{s}}$ nuclei are completely disintegrated into free nucleons. Disregarding the occurrence of $\alpha$ particles, in particular, is certainly an approximation 
which becomes invalid when the temperature drops below about $1 \mathrm{MeV}$, i.e., when the shock is at large radii, typically around $300 \mathrm{~km}$ (see Bethe 1993, 1995, 1996a-c, 1997). The presence of $\alpha$ particles reduces the neutrino heating, because electron neutrinos and antineutrinos are absorbed only on nucleons, but energy released by the recombination of $\alpha$ 's during shock expansion supports the shock at a later stage and contributes to the energy budget of the explosion. Since in the context of this paper we do not attempt to calculate the explosion energy, but are interested in a qualitative discussion of the revival phase of the stalled shock, the recombination of nucleons to $\alpha$ particles is probably not a crucial issue.

As will be demonstrated below, the optical depth between $R_{\mathrm{g}}$ and $R_{\mathrm{S}}$ is small such that $\int_{R_{\mathrm{g}}}^{R_{\mathrm{s}}} \mathrm{d} r\left\langle\kappa_{\mathrm{a}}\right\rangle /\left\langle\mu_{\nu}\right\rangle \lesssim 0.5$. Therefore the reabsorption probability of emitted neutrinos is also small and an approximation to the solution of Eq. (72) at the shock position is

$$
L_{\nu}\left(R_{\mathrm{S}}\right) \approx\left(1-\int_{R_{\mathrm{g}}}^{R_{\mathrm{S}}} \mathrm{d} r \frac{\left\langle\kappa_{\mathrm{a}}\right\rangle}{\left\langle\mu_{\nu}\right\rangle}\right) L_{\nu}\left(R_{\mathrm{g}}\right)+\int_{R_{\mathrm{g}}}^{R_{\mathrm{s}}} \mathrm{d} r 4 \pi r^{2} Q_{\nu}^{-}
$$

The net energy deposition is found to be

$$
\begin{aligned}
L_{\nu}\left(R_{\mathrm{g}}\right)-L_{\nu}\left(R_{\mathrm{s}}\right) & \approx \int_{R_{\mathrm{g}}}^{R_{\mathrm{s}}} \mathrm{d} r \frac{\left\langle\kappa_{\mathrm{a}}\right\rangle}{\left\langle\mu_{\nu}\right\rangle} L_{\nu}\left(R_{\mathrm{g}}\right)-\int_{R_{\mathrm{g}}}^{R_{\mathrm{s}}} \mathrm{d} r 4 \pi r^{2} Q_{\nu}^{-} \\
& \equiv \mathcal{H}-\mathcal{C} .
\end{aligned}
$$

Since in the layer bounded by $R_{\mathrm{g}}$ and $R_{\mathrm{s}}$ neutrino heating takes place, $L_{\nu}\left(R_{\mathrm{g}}\right) \geq L_{\nu}\left(R_{\mathrm{S}}\right)$ (this will also be verified below). Expanding the exponential damping factors only to lowerst order in the exponent implies a slight overestimation of the energy input into the stellar medium by neutrinos (because the luminosity entering at $R_{\mathrm{g}}$ is assumed to decay linearly through the layer), but also the energy loss by neutrinos is overestimated, because the reabsorption of emitted neutrinos is not included.

Using $Q_{\nu}^{+}$from Eq. (28) in Eq. (70), $L_{\nu}=2 L_{\nu_{\mathrm{e}}}$, and the density profile from Eq. (63), one finds for the first integral in Eq. (82):

$$
\begin{aligned}
\mathcal{H} \approx 4.910^{50} \frac{L_{\nu, 52}\left(R_{\mathrm{g}}\right)}{\left\langle\mu_{\nu}\right\rangle^{*}} & \left(k T_{\nu_{\mathrm{e}}, 4}\right)^{2} \rho_{\mathrm{s}, 9} R_{\mathrm{s}, 7} \\
\times & {\left[\left(\frac{R_{\mathrm{s}}}{R_{\mathrm{g}}}\right)^{2}-1\right]\left[\frac{\mathrm{erg}}{\mathrm{s}}\right], }
\end{aligned}
$$

where $k T_{\nu_{\mathrm{e}}}$ was again treated as a constant, $\left\langle\mu_{\nu}\right\rangle^{*}$ defines an average value of the flux factor in the layer between $R_{\mathrm{g}}$ and $R_{\mathrm{S}}$, and $\rho_{\mathrm{s}, 9}$ is the density behind the shock in units of $10^{9} \mathrm{~g} \mathrm{~cm}^{-3}$. The second integral in Eq. (82) is evaluated with $Q_{\nu}^{-}$from Eq. (31) and the temperature and density relations from Eq. (63):

$$
\begin{aligned}
\mathcal{C} \approx 2.910^{50}\left(k T_{\mathrm{s}, 2}\right)^{6} & \rho_{\mathrm{s}, 9} R_{\mathrm{s}, 7}^{3} \\
\times & {\left[\left(\frac{R_{\mathrm{s}}}{R_{\mathrm{g}}}\right)^{6}-1\right] \quad\left[\frac{\mathrm{erg}}{\mathrm{s}}\right] . }
\end{aligned}
$$

Employing the gain condition, Eq. (33), and again making use of Eq. (63), one gets

$$
\left(\frac{k T_{\mathrm{s}}}{2 \mathrm{MeV}}\right)^{6} \approx 0.55 \frac{L_{\nu, 52}\left(R_{\mathrm{g}}\right)}{\left\langle\mu_{\nu}\right\rangle_{\mathrm{g}} R_{\mathrm{g}, 7}^{2}}\left(\frac{k T_{\nu_{\mathrm{e}}}}{4 \mathrm{MeV}}\right)^{2}\left(\frac{R_{\mathrm{g}}}{R_{\mathrm{s}}}\right)^{6}
$$

which serves to rewrite Eq. (84) as

$$
\begin{aligned}
& \mathcal{C} \approx 1.610^{50} \frac{L_{\nu, 52}\left(R_{\mathrm{g}}\right)}{\left\langle\mu_{\nu}\right\rangle_{\mathrm{g}}}\left(k T_{\nu_{\mathrm{e}}, 4}\right)^{2} \rho_{\mathrm{s}, 9} R_{\mathrm{s}, 7} \\
& \times\left(\frac{R_{\mathrm{s}}}{R_{\mathrm{g}}}\right)^{2}\left[1-\left(\frac{R_{\mathrm{g}}}{R_{\mathrm{s}}}\right)^{6}\right]\left[\frac{\mathrm{erg}}{\mathrm{s}}\right] .
\end{aligned}
$$

Combining Eqs. (83) and (86) gives the net energy transfer to the stellar medium in the gain region:

$\mathcal{H}-\mathcal{C} \approx \mathcal{H} \times\left\{1-\frac{1}{3} \frac{\left\langle\mu_{\nu}\right\rangle^{*}}{\left\langle\mu_{\nu}\right\rangle_{\mathrm{g}}}\left[1+\left(\frac{R_{\mathrm{g}}}{R_{\mathrm{s}}}\right)^{2}+\left(\frac{R_{\mathrm{g}}}{R_{\mathrm{s}}}\right)^{4}\right]\right\}$

Since $\left\langle\mu_{\nu}\right\rangle^{*} /\left\langle\mu_{\nu}\right\rangle_{\mathrm{g}} \sim 1$ and $R_{\mathrm{s}}>R_{\mathrm{g}}$, typically $R_{\mathrm{s}} \sim 2 R_{\mathrm{g}}$, we verify that $\mathcal{H}-\mathcal{C}>0$ and therefore $L_{\nu}\left(R_{\mathrm{s}}\right)<L_{\nu}\left(R_{\mathrm{g}}\right)$, as expected for the neutrino heating region. For $\beta=$ $\rho_{\mathrm{s}} / \rho_{\mathrm{p}} \approx 7$ (Eq. (41)) and $\alpha=1 / \sqrt{2}$, Eq. (44) yields for the postshock density

$\rho_{\mathrm{s}} \approx 310^{9} \frac{(-\dot{M})}{M_{\odot} / \mathrm{s}}\left(\frac{\widetilde{M}}{M_{\odot}}\right)^{-1 / 2} R_{\mathrm{s}, 7}^{-3 / 2}\left[\frac{\mathrm{g}}{\mathrm{cm}^{3}}\right]$.

Using this and $R_{\mathrm{s}} \sim 2 R_{\mathrm{g}}$ in Eq. (83) leads to

$$
\begin{aligned}
\int_{R_{\mathrm{g}}}^{R_{\mathrm{s}}} \mathrm{d} r \frac{\left\langle\kappa_{\mathrm{a}}\right\rangle}{\left\langle\mu_{\nu}\right\rangle} & =\frac{\mathcal{H}}{L_{\nu}\left(R_{\mathrm{g}}\right)} \\
& \sim \frac{0.44}{\left\langle\mu_{\nu}\right\rangle^{*}} \frac{\left(k T_{\nu_{\mathrm{e}}, 4}\right)^{2}}{R_{\mathrm{s}, 7}^{1 / 2}} \frac{(-\dot{M})}{M_{\odot} / \mathrm{s}}\left(\frac{\widetilde{M}}{M_{\odot}}\right)^{-\frac{1}{2}}
\end{aligned}
$$

For sufficiently small accretion rates $|\dot{M}|$ this is less than about 0.5 , and the assumption made before Eq. (81) is verified, i.e., the reabsorption of neutrinos emitted in the gain region can be neglected.

\section{Mass accretion onto the neutron star}

The shock accretes mass at a rate $\dot{M} \equiv 4 \pi R_{\mathrm{s}}^{2} \rho_{\mathrm{p}} v_{\mathrm{p}}$ as determined by the conditions in the core of the progenitor star (see Sect. 4.4). In a stationary state, this rate is equal to the rate at which matter is advected inward from the shock to the neutrinosphere to be finally added into the neutron star. The rate at which matter can be absorbed by the neutron star, however, depends on the efficiency by which neutrinos are able to remove the energy excess of the infalling material relative to the energy of the strongly bound matter in the neutron star surface layers. For the large accretion rates typical of the collapsed stellar core right after bounce, the density is so high that the infalling matter becomes opaque to neutrinos. In this case the efficiency of the energy loss is reduced. When the gas is 
hotter, the neutrino opacity increases (because of the energy dependence of the neutrino cross sections), and the neutrinosphere moves to a larger radius. Due to this regulatory effect, the neutrinospheric temperature is a rather inert quantity and, e.g., turns out to be very similar in different numerical models. Therefore it is not a steadystate mass accretion rate which governs the temperature at the base of the "atmosphere" (as for accretion in optically thin conditions), but the "surface" of the nascent neutron star forms where the temperature is sufficiently high for neutrino opaqueness to set in.

When neutrino cooling is not efficient enough, the advection of matter through the neutrino cooling region is reduced compared to the accretion into the shock, and matter piles up on top of the neutron star. Similarly, strong neutrino heating in the gain region can reduce the inflow of matter. The transition from accretion to an explosion is characterized by an inversion of infall to outflow. For this reason the analysis of the conditions for shock revival requires the inclusion of this sort of time-dependence in the discussion. In the simplified model considered here, the mass accretion rate is allowed to change between $R_{\mathrm{s}}$ and $R_{\mathrm{g}}$. Matter advected through $R_{\mathrm{g}}$ at a rate determined by the efficiency of neutrino cooling is then assumed to be added into the neutron star (compare Fig. 2).

Using Eqs. (2) and (6) and the definition $\dot{M}(r)=$ $4 \pi r^{2} \rho v$, Eq. (4) can be rewritten in the following form:

$$
\begin{aligned}
\frac{\partial}{\partial r}\left[\dot{M}\left(\frac{e+P}{\rho}-\frac{G \widetilde{M}}{r}\right)\right]= & 4 \pi r^{2}\left(Q_{\nu}+Q_{\mathrm{d}}\right) \\
& -4 \pi r^{2} \rho \frac{\partial}{\partial t}\left(\frac{e}{\rho}\right) \\
& +\left(\frac{e}{\rho}-\frac{G \widetilde{M}}{r}\right) \frac{\partial \dot{M}}{\partial r}
\end{aligned}
$$

where $Q_{\nu}=Q_{\nu}^{+}-Q_{\nu}^{-}$is the net rate (per unit volume) of energy transfer between neutrinos and the stellar medium and $Q_{\mathrm{d}}$ denotes the energy consumed or released by the photodisintegration of nuclei. The latter term has to be introduced in the equation when rest-mass contributions from nucleons and nuclei are not included in the internal energy density $\varepsilon$ (Eq. (5)). The nuclei present in the accretion flow through the shock are assumed to be dissociated to free nucleons within the shock front (cf. Eq. (38)). Therefore the rate $Q_{\mathrm{d}}$ in terms of the (positive) nuclear binding energy per unit mass, $q_{\mathrm{d}}$, is

$Q_{\mathrm{d}}=\rho v q_{\mathrm{d}} \delta\left(r-R_{\mathrm{s}}\right)$.

Here $\delta(x)$ is the delta function. For $v<0$, which is true in case of accretion, energy is extracted from the stellar medium, i.e., $Q_{\mathrm{d}}<0$. Now integrating Eq. (90) between
$R_{\nu}$ and a radius $r$ that is infinitesimally larger than $R_{\mathrm{S}}$ gives

$$
\begin{gathered}
\dot{M}\left[\frac{e+P}{\rho}-\frac{G \widetilde{M}}{r}\right]_{R_{\mathrm{s}}}-\dot{M}^{\prime}\left[\frac{e+P}{\rho}-\frac{G \widetilde{M}}{r}\right]_{R_{\nu}}= \\
\dot{M}^{\prime} q_{\mathrm{d}}+\int_{M\left(R_{\nu}\right)}^{M\left(R_{\mathrm{s}}\right)} \mathrm{d} M\left[\frac{Q_{\nu}}{\rho}-\frac{\partial}{\partial t}\left(\frac{e}{\rho}\right)\right] \\
+\int_{\dot{M}^{\prime}}^{\dot{M}} \mathrm{~d} \dot{M}\left(q_{\mathrm{d}}+\frac{e}{\rho}-\frac{G \widetilde{M}}{r}\right),
\end{gathered}
$$

where $\partial M / \partial r=4 \pi r^{2} \rho$ was used. The mass accretion rate through the shock was defined as $\dot{M}=4 \pi R_{\mathrm{s}}^{2} \rho_{\mathrm{p}} v_{\mathrm{p}}$ and the corresponding accretion rate through the neutrinosphere as $\dot{M}^{\prime} \equiv \dot{M}\left(R_{\nu}\right)=4 \pi R_{\nu}^{2} \rho_{\nu} v_{\nu}$. The term for the rate of energy consumption by nuclear dissociation was split into two parts according to $\dot{M}(r)=\dot{M}^{\prime}+\int_{\dot{M}^{\prime}}^{\dot{M}(r)} \mathrm{d} \dot{M}$.

From Eq. (92) an approximation for $\dot{M}^{\prime}$ can be derived by taking into account that $\left|Q_{\nu} / \rho\right| \gg \partial(e / \rho) / \partial t$ in the region between $R_{\nu}$ and $R_{\mathrm{s}}$, where strong neutrino heating and cooling occurs. Moreover, the integrand of the last term on the right hand side of Eq. (92) is usually small, because $q_{\mathrm{d}}$ corresponds to about 8-9 MeV per nucleon for complete disintegration of nuclei into free nucleons, $G \widetilde{M} / R_{\mathrm{s}} \sim 14\left(\widetilde{M} / M_{\odot}\right) / R_{\mathrm{s}, 7} \mathrm{MeV}$ per nucleon, and $e / \rho \approx \frac{1}{2} v_{\mathrm{p}}^{2} \sim \frac{1}{2} G \widetilde{M} / R_{\mathrm{S}}$ immediately above the shock, where the infall velocity $v_{\mathrm{p}}$ is given by Eq. (43) and the specific internal energy is typically much smaller than the specific kinetic energy. For the same reason, the first term on the left hand side of Eq. (92) is much smaller than the second term when $\dot{M}$ and $\dot{M}^{\prime}$ are of the same order. With all this one gets

$\dot{M}^{\prime} \approx-\int_{R_{\nu}}^{R_{\mathrm{s}}} \mathrm{d} r 4 \pi r^{2} Q_{\nu} \times\left(\left[\frac{e+P}{\rho}\right]_{R_{\nu}}-\frac{G \widetilde{M}}{R_{\nu}}+q_{\mathrm{d}}\right)^{-1}$

Because of the large gravitational binding energy of matter at the neutrinosphere, the term in brackets in Eq. (93) is negative. The integral adds up the contributions from neutrino cooling between $R_{\nu}$ and $R_{\mathrm{g}}$ and from neutrino heating between $R_{\mathrm{g}}$ and $R_{\mathrm{s}}$. If cooling is stronger (which is the case in the first second after bounce), the integral is negative and $\dot{M}^{\prime}<0$, i.e., the neutron star accretes matter. If neutrino heating dominates, there is mass outflow, $\dot{M}^{\prime}>0$.

Such mass loss takes place during the later phase of the neutrino-cooling evolution of the nascent neutron star, where a baryonic wind, the so-called neutrino-driven wind, is blown off the neutron star surface due to neutrino energy deposition just outside the neutrinosphere (Qian \& Woosley 1996). The transition from accretion to mass outflow and the onset of mass loss can be discussed with the formulae presented here. A description of the wind regime (where the fluid velocity $v$ approaches the local speed of sound), however, is beyond the scope of the present work, 
because it requires retaining the velocity gradient in the momentum equation, Eq. (3). Assuming steady-state conditions, this leads to the well known set of dynamic wind equations which can also be discussed by analytic means (see Qian \& Woosley 1996, and references therein). In contrast, the toy model developed in this paper does not make use of steady-state assumptions for the mass flow through the gain layer, i.e., it is allowed that $\dot{M} \neq \dot{M}^{\prime}$ in general.

The integral in Eq. (93) was evaluated in Sect. 6:

$\int_{R_{\nu}}^{R_{\mathrm{s}}} \mathrm{d} r 4 \pi r^{2} Q_{\nu}=-L_{\mathrm{acc}}+\mathcal{H}-\mathcal{C}$.

Equation (77) gives the net energy exchange between neutrinos and stellar medium in the layer $\left[R_{\nu}, R_{\mathrm{g}}\right]$, Eq. (87) the corresponding result for the interval $\left[R_{\mathrm{g}}, R_{\mathrm{s}}\right]$, when $\mathcal{H}$ is taken from Eq. (83) and the neutrino luminosity $L_{\nu}\left(R_{\mathrm{g}}\right)$ from Eq. (76) with $a$ and $b$ provided by Eqs. (74) and (75), respectively. Plugging in numbers representative for the early post-bounce evolution, $L_{\nu} \approx$ $510^{52} \mathrm{erg} \mathrm{s}^{-1}, R_{\nu} \approx 50 \mathrm{~km}, \widetilde{M} \approx 1 M_{\odot}, a \sim 1$, one finds $L_{\nu}\left(R_{\mathrm{g}}\right) \approx 6.310^{52} \mathrm{erg} \mathrm{s}^{-1}$ and $\int_{R_{\nu}}^{R_{\mathrm{g}}} \mathrm{d} r 4 \pi r^{2} Q_{\nu}=$ $-L_{\mathrm{acc}}=L_{\nu}\left(R_{\nu}\right)-L_{\nu}\left(R_{\mathrm{g}}\right) \approx-1.310^{52} \mathrm{erg} \mathrm{s}^{-1}$, and us$\operatorname{ing} R_{\mathrm{s}} \approx 2 R_{\mathrm{g}} \approx 200 \mathrm{~km},\left\langle\mu_{\nu}\right\rangle^{*} \sim 1,\left\langle\mu_{\nu}\right\rangle_{\mathrm{g}} \sim 0.75$, yields $\int_{R_{\mathrm{g}}}^{R_{\mathrm{s}}} \mathrm{d} r 4 \pi r^{2} Q_{\nu}=\mathcal{H}-\mathcal{C} \approx 7.710^{51} \mathrm{erg} \mathrm{s}^{-1}$. The gravitational energy at the neutrinosphere at $50 \mathrm{~km}$ is about $-28 \mathrm{MeV}$ per nucleon, $q_{\mathrm{d}}$ is roughly $8 \mathrm{MeV}$ per nucleon, and the internal energy plus pressure account for typically $\sim 10 \mathrm{MeV}$ per nucleon:

$$
\left(\frac{e+P}{\rho}\right)_{R_{\nu}} \approx\left(\frac{5}{2}+\frac{4}{3} Y_{\mathrm{e}} \frac{\mathcal{F}_{3}\left(\eta_{\mathrm{e}}\right)}{\mathcal{F}_{2}\left(\eta_{\mathrm{e}}\right)}\right) \frac{k T_{\nu}}{m_{\mathrm{u}}} \approx \frac{7}{2} \frac{k T_{\nu}}{m_{\mathrm{u}}},
$$

where $e=\varepsilon$ has been applied because $\frac{1}{2} \rho v^{2} \ll \varepsilon$ at the neutrinosphere. Therefore the sum of the terms in the denominator of Eq. (93) can be estimated to be about $-10^{19} \mathrm{erg} \mathrm{g}^{-1}$. This leads to a mass accretion rate of the neutron star of $\dot{M}^{\prime} \sim-0.3 M_{\odot} \mathrm{s}^{-1}$, a value which is in the range of the results of detailed numerical simulations and is of the order of the mass infall rate on the shock, $\dot{M}$.

\section{Mass and energy conservation in the gain region}

Mass and energy conservation in the gain region between $R_{\mathrm{g}}$ and $R_{\mathrm{s}}$ determine the early postbounce evolution of the supernova shock. For example, the shock is pushed outward when the matter that falls through the shock stays hot and piles up on top of the neutron star, forming an extended envelope instead of being accreted into the dense core quickly after efficient energy loss in the neutrino cooling layer below $R_{\mathrm{g}}$. Similarly, strong neutrino heating in the gain region causes an increase of the postshock pressure and thus drives an expansion of the shock. On the other hand, enhanced neutrino emission will extract mass and/or energy from the layer which supports the supernova shock. The consequence will be a retraction of the shock in radius. These effects need to be accounted for by an appropriate discussion of the delayed explosion mechanism. A steady-state picture is certainly not adequate.

\subsection{Mass in the gain region}

The mass $\Delta M_{\mathrm{g}}$ in the gain region can be calculated as volume integral over the density:

$\Delta M_{\mathrm{g}}=\int_{R_{\mathrm{g}}}^{R_{\mathrm{s}}} \mathrm{d} r 4 \pi r^{2} \rho(r)$

with the density $\rho(r)$ given by Eq. (59). Alternatively, since the latter equation is the exact solution for hydrostatic equilibrium, one can use $\rho(r)=-r^{2}(\mathrm{~d} P / \mathrm{d} r) /(G \widetilde{M})$ with $P(r)$ from Eq. (60). Defining the coefficients $c_{1} \equiv$ $\rho_{\mathrm{s}}^{1 / 3}-G \widetilde{M} /\left(4 K R_{\mathrm{s}}\right)$ and $c_{2} \equiv G \widetilde{M} /(4 K)$, one finds:

$$
\begin{aligned}
\Delta M_{\mathrm{g}}= & 4 \pi\left[\frac{1}{3} c_{1}^{3}\left(R_{\mathrm{s}}^{3}-R_{\mathrm{g}}^{3}\right)+\frac{3}{2} c_{1}^{2} c_{2}\left(R_{\mathrm{s}}^{2}-R_{\mathrm{g}}^{2}\right)\right. \\
& \left.+3 c_{1} c_{2}^{2}\left(R_{\mathrm{s}}-R_{\mathrm{g}}\right)+c_{2}^{3} \ln \left(\frac{R_{\mathrm{s}}}{R_{\mathrm{g}}}\right)\right] \\
= & 4 \pi\left[\frac{1}{3}\left(R_{\mathrm{s}}^{3} \rho_{\mathrm{s}}-R_{\mathrm{g}}^{3} \rho_{\mathrm{g}}\right)+\frac{c_{2}}{2}\left(R_{\mathrm{s}}^{2} \rho_{\mathrm{s}}^{\frac{2}{3}}-R_{\mathrm{g}}^{2} \rho_{\mathrm{g}}^{\frac{2}{3}}\right)\right. \\
& \left.+c_{2}^{2}\left(R_{\mathrm{s}} \rho_{\mathrm{s}}^{\frac{1}{3}}-R_{\mathrm{g}} \rho_{\mathrm{g}}^{\frac{1}{3}}\right)+c_{2}^{3} \ln \left(\frac{R_{\mathrm{s}}}{R_{\mathrm{g}}}\right)\right]
\end{aligned}
$$

In deriving the second form of Eq. (97), use was made of $\rho(r)=\left(c_{1}+c_{2} / r\right)^{3}$. Moreover, with $\rho=(P / K)^{3 / 4}$ the density in Eq. (97) can be substituted by the pressure $P$. Note that the quantities $\rho_{\mathrm{g}}=\rho\left(R_{\mathrm{g}}\right)$ and $P_{\mathrm{g}}=P\left(R_{\mathrm{g}}\right)$ at the gain radius must be expressed by the exact relations of Eqs. (59) and (60), respectively. They depend on the postshock state of the matter as do the coefficients $c_{1}$ and $c_{2}$. The gain radius $R_{\mathrm{g}}$ is given by Eq. (66). It is also a function of the conditions immediately behind the shock. Writing the postshock temperature in terms of the postshock pressure via Eq. (56), $k T_{\mathrm{s}}=\left[3 P_{\mathrm{s}} /\left(f_{\mathrm{r}} g_{\mathrm{r}} a_{\gamma}\right)\right]^{1 / 4}$, and using Eqs. (39), (43), (44) and (53)-(55) with typical values $\beta \sim 7, \alpha \sim 1 / \sqrt{2}, s_{\gamma} \sim 4$, and $\eta_{\mathrm{e}} \sim 2$ (the exact values of these parameters are not essential for the discussion and affect the result rather insensitively), one gets in case of $\left|U_{\mathrm{s}}\right|=\left|\dot{R}_{\mathrm{s}}\right| \ll\left|v_{\mathrm{p}}\right|:$

$k T_{\mathrm{s}} \approx 2 R_{\mathrm{s}, 7}^{-\frac{5}{8}}\left(\frac{-\dot{M}}{M_{\odot} / \mathrm{s}}\right)^{\frac{1}{4}}\left(\frac{\widetilde{M}}{M_{\odot}}\right)^{\frac{1}{8}}[\mathrm{MeV}]$.

Inserting this in Eq. (66) and using $L_{\nu}=2 L_{\nu_{\mathrm{e}}}$ yields

$$
\begin{aligned}
R_{\mathrm{g}, 7} \approx 1.13 R_{\mathrm{s}, 7}^{\frac{9}{16}}\left(k T_{\nu_{\mathrm{e}}, 4}\right)^{-\frac{1}{2}} & \left(\frac{L_{\nu, 52}\left(R_{\mathrm{g}}\right)}{\left\langle\mu_{\nu}\right\rangle_{\mathrm{g}}}\right)^{-\frac{1}{4}} \\
& \times\left(\frac{-\dot{M}}{M_{\odot} / \mathrm{s}}\right)^{\frac{3}{8}}\left(\frac{\widetilde{M}}{M_{\odot}}\right)^{\frac{3}{16}}
\end{aligned}
$$

where the neutrino luminosity at the gain radius, $L_{\nu}\left(R_{\mathrm{g}}\right)$, is given by Eq. (76).

Instead of the exact expression of Eq. (97) an approximation for $\Delta M_{\mathrm{g}}$ is sometimes preferable. Performing the 
integration of Eq. (96) with the approximate density profile of Eq. (63), one finds

$\Delta M_{\mathrm{g}} \approx 4 \pi \rho_{\mathrm{s}} R_{\mathrm{s}}^{3} \ln \left(\frac{R_{\mathrm{s}}}{R_{\mathrm{g}}}\right) \propto \frac{-\dot{M}}{\widetilde{M}^{1 / 2}} R_{\mathrm{s}}^{3 / 2} \ln \left(\frac{R_{\mathrm{s}}}{R_{\mathrm{g}}}\right)$.

Here $\rho_{\mathrm{s}}$ was written in terms of $R_{\mathrm{s}}$ by making use of $\rho_{\mathrm{s}}=$ $\beta \rho_{\mathrm{p}}$ and Eq. (44). Moreover, from Eq. (99) one can deduce that $R_{\mathrm{S}} / R_{\mathrm{g}} \propto R_{\mathrm{s}}^{7 / 16}$ for $\left|U_{\mathrm{s}}\right| \ll\left|v_{\mathrm{p}}\right|$. An increase of the shock radius therefore means that $\Delta M_{\mathrm{g}}$ will also grow.

The rate at which the mass in the gain region changes in time due to a shift of the upper and lower boundaries of this region but also due to a variation of the density of the stellar medium, is determined as the total time derivative of Eq. (96):

$\frac{\mathrm{d}}{\mathrm{d} t}\left(\Delta M_{\mathrm{g}}\right)=4 \pi R_{\mathrm{s}}^{2} \rho_{\mathrm{s}} \dot{R}_{\mathrm{s}}-4 \pi R_{\mathrm{g}}^{2} \rho_{\mathrm{g}} \dot{R}_{\mathrm{g}}+\int_{R_{\mathrm{g}}}^{R_{\mathrm{s}}} \mathrm{d} r 4 \pi r^{2} \frac{\partial \rho}{\partial t}$,

where $\dot{R}_{\mathrm{s}} \equiv \mathrm{d} R_{\mathrm{s}} / \mathrm{d} t=U_{\mathrm{s}}$ is the shock velocity and $\dot{R}_{\mathrm{g}} \equiv \mathrm{d} R_{\mathrm{g}} / \mathrm{d} t$ the velocity of the gain radius. When the integration in Eq. (101) is carried out to a radius infinitesimally smaller than $R_{\mathrm{s}}$ with the help of Eq. (2), one obtains

$$
\begin{aligned}
\frac{\mathrm{d}}{\mathrm{d} t}\left(\Delta M_{\mathrm{g}}\right) & =4 \pi R_{\mathrm{s}}^{2} \rho_{\mathrm{s}}\left(\dot{R}_{\mathrm{s}}-v_{\mathrm{s}}\right)-4 \pi R_{\mathrm{g}}^{2} \rho_{\mathrm{g}}\left(\dot{R}_{\mathrm{g}}-v_{\mathrm{g}}\right) \\
& =4 \pi R_{\mathrm{s}}^{2} \rho_{\mathrm{p}} \dot{R}_{\mathrm{s}}-4 \pi R_{\mathrm{g}}^{2} \rho_{\mathrm{g}} \dot{R}_{\mathrm{g}}-\dot{M}+\dot{M}^{\prime},
\end{aligned}
$$

with $v_{\mathrm{g}}$ and $v_{\mathrm{s}}$ being the velocities of the stellar medium at the gain radius and just behind the shock, respectively. The lower expression was derived by using the shock jump condition for the mass flow, Eq. (36), and the definitions $\dot{M}=4 \pi R_{\mathrm{s}}^{2} \rho_{\mathrm{p}} v_{\mathrm{p}}$ and $\dot{M}^{\prime}=4 \pi R_{\mathrm{g}}^{2} \rho_{\mathrm{g}} v_{\mathrm{g}}=4 \pi R_{\nu}^{2} \rho_{\nu} v_{\nu}$ as introduced in Sect. 7. Equation (102) shows that the mass in the gain region can change because of inflow and outflow of gas but also due to the motion of the boundaries $R_{\mathrm{g}}$ and $R_{\mathrm{s}}$. Knowing the initial mass in this layer, $\Delta M_{\mathrm{g}}^{0}$, Eq. (102) allows one to calculate the value at later times.

\subsection{Energy in the gain region}

Since the postshock matter is effectively in hydrostatic equilibrium (see Sect. 5) the kinetic energy is negligible compared to the internal energy and the gravitational potential energy, and the total energy in the gain region is therefore given by

$\Delta E_{\mathrm{g}}=\int_{R_{\mathrm{g}}}^{R_{\mathrm{s}}} \mathrm{d} r 4 \pi r^{2}\left[\varepsilon(r)-\frac{G \widetilde{M}}{r} \rho(r)\right]$.

To evaluate the right hand side, one substitutes $\varepsilon=$ $P /(\Gamma-1)$, which relates the internal energy density $\varepsilon$ and the pressure $P$ for an ideal gas, with the adiabatic index $\Gamma$ being typically between $4 / 3$ and $5 / 3$, depending on whether relativistic or nonrelativistic particles, respectively, dominate the pressure. In addition making use of hydrostatic equilibrium (Eq. (47)) or, alternatively, applying the virial theorem, one finds

$$
\begin{aligned}
\Delta E_{\mathrm{g}}=\frac{4 \pi}{3(\Gamma-1)} & \left(P_{\mathrm{s}} R_{\mathrm{s}}^{3}-P_{\mathrm{g}} R_{\mathrm{g}}^{3}\right) \\
& -4 \pi G \widetilde{M} \frac{3 \Gamma-4}{3(\Gamma-1)} \int_{R_{\mathrm{g}}}^{R_{\mathrm{s}}} \mathrm{d} r r \rho(r) .
\end{aligned}
$$

The second term is the gravitational potential energy times $\left(\Gamma-\frac{4}{3}\right) /(\Gamma-1)$. An exact expression for the integral is obtained when Eq. (59) is used for $\rho(r)$ :

$$
\begin{aligned}
\int_{R_{\mathrm{g}}}^{R_{\mathrm{s}}} \mathrm{d} r r \rho(r)=\frac{c_{1}^{3}}{2}\left(R_{\mathrm{s}}^{2}-R_{\mathrm{g}}^{2}\right) & +\left(3 c_{1}^{2} c_{2}+\frac{c_{2}^{3}}{R_{\mathrm{s}} R_{\mathrm{g}}}\right)\left(R_{\mathrm{s}}-R_{\mathrm{g}}\right) \\
& +3 c_{1} c_{2}^{2} \ln \left(\frac{R_{\mathrm{s}}}{R_{\mathrm{g}}}\right)
\end{aligned}
$$

The coefficients $c_{1}$ and $c_{2}$ were already defined in Sect. 8.1. For the following discussion an approximation of this integral is sufficient. It can be derived by employing the approximate power law profile for the density, Eq. (63):

$\int_{R_{\mathrm{g}}}^{R_{\mathrm{s}}} \mathrm{d} r r \rho(r) \approx \rho_{\mathrm{s}} \frac{R_{\mathrm{s}}^{2}}{R_{\mathrm{g}}}\left(R_{\mathrm{s}}-R_{\mathrm{g}}\right)$.

The rate at which the total energy in the gain region changes with time can be calculated as the time derivative of Eq. (103). With the definition $l \equiv(\varepsilon+P) / \rho-G \widetilde{M} / r$ one finds

$$
\begin{aligned}
\frac{\mathrm{d}}{\mathrm{d} t}\left(\Delta E_{\mathrm{g}}\right)= & 4 \pi R_{\mathrm{s}}^{2} \rho_{\mathrm{s}} l_{\mathrm{s}} \dot{R}_{\mathrm{s}}-4 \pi R_{\mathrm{g}}^{2} \rho_{\mathrm{g}} l_{\mathrm{g}} \dot{R}_{\mathrm{g}} \\
& -4 \pi R_{\mathrm{s}}^{2} P_{\mathrm{s}} \dot{R}_{\mathrm{s}}+4 \pi R_{\mathrm{g}}^{2} P_{\mathrm{g}} \dot{R}_{\mathrm{g}} \\
& +\int_{R_{\mathrm{g}}}^{R_{\mathrm{s}}} \mathrm{d} r 4 \pi r^{2}\left(\frac{\partial \varepsilon}{\partial t}-\frac{G \widetilde{M}}{r} \frac{\partial \rho}{\partial t}\right)
\end{aligned}
$$

where $\dot{R}_{\mathrm{s}}$ and $\dot{R}_{\mathrm{g}}$ have the same meaning as in Eq. (101). The partial derivatives in the integral can be substituted by Eqs. (2) and (4). Making additional use of Eqs. (5) and (6) and of $\frac{1}{2} \rho v^{2} \ll \varepsilon$ yields

$$
\begin{aligned}
\frac{\mathrm{d}}{\mathrm{d} t}\left(\Delta E_{\mathrm{g}}\right)= & 4 \pi R_{\mathrm{s}}^{2} \rho_{\mathrm{s}} l_{\mathrm{s}}\left(\dot{R}_{\mathrm{s}}-v_{\mathrm{s}}\right)-4 \pi R_{\mathrm{g}}^{2} \rho_{\mathrm{g}} l_{\mathrm{g}}\left(\dot{R}_{\mathrm{g}}-v_{\mathrm{g}}\right) \\
& -4 \pi R_{\mathrm{s}}^{2} P_{\mathrm{s}} \dot{R}_{\mathrm{s}}+4 \pi R_{\mathrm{g}}^{2} P_{\mathrm{g}} \dot{R}_{\mathrm{g}}+\int_{R_{\mathrm{g}}}^{R_{\mathrm{s}}} \mathrm{d} r 4 \pi r^{2} Q_{\nu}
\end{aligned}
$$

Now employing the continuity equation for the mass flow across the shock, Eq. (36), and replacing the integral for the energy exchange with neutrinos between $R_{\mathrm{g}}$ and $R_{\mathrm{s}}$ by $\mathcal{H}-\mathcal{C}$ as given in Eqs. (82) and (87), one ends up with

$$
\begin{aligned}
\frac{\mathrm{d}}{\mathrm{d} t}\left(\Delta E_{\mathrm{g}}\right)= & 4 \pi R_{\mathrm{s}}^{2} \rho_{\mathrm{p}} l_{\mathrm{s}}\left(\dot{R}_{\mathrm{s}}-v_{\mathrm{p}}\right)-4 \pi R_{\mathrm{g}}^{2} \rho_{\mathrm{g}} l_{\mathrm{g}}\left(\dot{R}_{\mathrm{g}}-v_{\mathrm{g}}\right) \\
& -4 \pi R_{\mathrm{s}}^{2} P_{\mathrm{s}} \dot{R}_{\mathrm{s}}+4 \pi R_{\mathrm{g}}^{2} P_{\mathrm{g}} \dot{R}_{\mathrm{g}}+\mathcal{H}-\mathcal{C} \\
= & 4 \pi R_{\mathrm{s}}^{2} \rho_{\mathrm{p}} l_{\mathrm{s}} \dot{R}_{\mathrm{s}}-4 \pi R_{\mathrm{g}}^{2} \rho_{\mathrm{g}} l_{\mathrm{g}} \dot{R}_{\mathrm{g}}-4 \pi R_{\mathrm{s}}^{2} P_{\mathrm{s}} \dot{R}_{\mathrm{s}} \\
& +4 \pi R_{\mathrm{g}}^{2} P_{\mathrm{g}} \dot{R}_{\mathrm{g}}-\dot{M} l_{\mathrm{s}}+\dot{M}^{\prime} l_{\mathrm{g}}+\mathcal{H}-\mathcal{C} .
\end{aligned}
$$


The mass accretion rates $\dot{M}$ and $\dot{M}^{\prime}$ account for the inflow of matter into the gain region through the shock and for the mass that is advected through the gain radius, respectively (see Sect. 7 and discussion after Eq. (102)). Equation (109) means that the total energy in the gain region changes due to active mass motions, $p \mathrm{~d} V$ work associated with these mass motions, the movement of the boundaries, and neutrino heating.

Making use of $\varepsilon_{\mathrm{s}}=P_{\mathrm{s}} /(\Gamma-1), \rho_{\mathrm{s}} / \rho_{\mathrm{p}}=\beta$, and Eq. (39), one finds for $l_{\mathrm{s}}=\left(\varepsilon_{\mathrm{s}}+P_{\mathrm{s}}\right) / \rho_{\mathrm{s}}-G \widetilde{M} / R_{\mathrm{s}}$ :

$l_{\mathrm{s}} \approx-\left[1-\frac{\Gamma}{\Gamma-1} \frac{\beta-1}{\beta^{2}}\left(1-\frac{U_{\mathrm{s}}}{v_{\mathrm{p}}}\right)^{2}\right] \frac{G \widetilde{M}}{R_{\mathrm{s}}}$,

where Eq. (43) with $\alpha \approx 1 / \sqrt{2}$ was employed for $v_{\mathrm{p}}^{2} \approx$ $G \widetilde{M} / R_{\mathrm{s}} \approx 1.310^{19} R_{\mathrm{s}, 7}^{-1}\left(\widetilde{M} / M_{\odot}\right) \operatorname{erg~g}^{-1}$. Because of hydrostatic equilibrium a simple relation exists between $l_{\mathrm{g}}$ and $l_{\mathrm{s}}$. With $\varepsilon=P /(\Gamma-1)$ and Eqs. (56) and (59) one obtains

$l_{\mathrm{g}}=l_{\mathrm{s}}-\frac{3 \Gamma-4}{4(\Gamma-1)} \frac{G \widetilde{M}}{R_{\mathrm{s}}}\left(\frac{R_{\mathrm{s}}}{R_{\mathrm{g}}}-1\right)$.

Using the more general density-pressure relation $P=K \rho^{\gamma}$ instead of Eq. (56), and the corresponding hydrostatic density profile of Eq. (62), leads to

$l_{\mathrm{g}}=l_{\mathrm{s}}-\left(1-\frac{\Gamma}{\Gamma-1} \frac{\gamma-1}{\gamma}\right) \frac{G \widetilde{M}}{R_{\mathrm{s}}}\left(\frac{R_{\mathrm{s}}}{R_{\mathrm{g}}}-1\right)$.

For $\Gamma=\gamma$, this gives $l_{\mathrm{g}}=l_{\mathrm{s}}$.

\section{Evolution of shock radius and shock velocity}

The model developed in the preceding sections allows one to study the behavior of the supernova shock in response to the processes that play a role in the collapsed stellar core. The physics between the neutron star surface and the shock is constrained by the energy influx from the neutrinosphere on the one hand and the mass accretion into the shock front on the other. Equations (97), (104) (in combination with (105)), and (39) determine the shock radius $R_{\mathrm{s}}$, the shock velocity $U_{\mathrm{s}}$, and the postshock pressure $P_{\mathrm{s}}$. The state of the matter immediately behind the shock and that at the gain radius are related via Eqs. (59)(61) and (111), the gain radius $R_{\mathrm{g}}$ is given by Eq. (66), the postshock temperature by $k T_{\mathrm{s}}=\left[3 P_{\mathrm{s}} /\left(f_{\mathrm{r}} g_{\mathrm{r}} a_{\gamma}\right)\right]^{1 / 4}$ (Eq. (56)), and the postshock density as $\rho_{\mathrm{s}}=\beta \rho_{\mathrm{p}}$ with $\rho_{\mathrm{p}}$ from Eq. (44).

The mass accretion rate $\dot{M}$ into the shock is a fixed parameter of the problem (in Eq. (46) it is expressed in terms of the constant $H$ which is linked to the structure of the progenitor star). The rate of mass advection into the neutron star, $\dot{M}^{\prime}$, can be calculated from Eq. (93). The radius $R_{\nu}$ and mass $M$ of the neutron star, the neutrinospheric luminosity $L_{\nu}$, and the spectral temperature of the emitted electron neutrinos $T_{\nu_{\mathrm{e}}}$ (assumed to be roughly equal to the temperature $T_{\nu}$ of the stellar gas at the neutrinosphere) are also input parameters. The discussion takes into account the effects of neutrino losses in the cooling region, expressed by Eqs. (74)-(77), and of neutrino heating in the gain region as given by Eqs. (82), (83), and (87).

The time dependence of the considered model requires as initial conditions the values $\Delta M_{\mathrm{g}}^{0}$ and $\Delta E_{\mathrm{g}}^{0}$ for the initial mass and energy in the gain region. This couples the subsequent evolution in $\Delta M_{\mathrm{g}}$ and $\Delta E_{\mathrm{g}}$, which can be followed with Eqs. (102) and (109), respectively, to the situation that exists right after core bounce. Knowing $\dot{M}^{\prime}(t)$ allows one to include also the changes of the neutron star mass.

\subsection{Shock expansion and acceleration}

Combining Eqs. (104) and (106) and using Eq. (60) for $P_{\mathrm{g}}$ in terms of $P_{\mathrm{s}}$ with $K^{3 / 4}=P_{\mathrm{s}}^{3 / 4} / \rho_{\mathrm{s}}$, one gets the relation

$$
\begin{aligned}
\left(x-x_{0}\right) x^{3} \approx\left(\frac{R_{\mathrm{g}}}{R_{\mathrm{s}}}\right)^{3}[x & \left.+\left(\frac{R_{\mathrm{s}}}{R_{\mathrm{g}}}-1\right)\right]^{4} \\
& +4(3 \Gamma-4)\left(\frac{R_{\mathrm{s}}}{R_{\mathrm{g}}}-1\right) x^{3} .
\end{aligned}
$$

Here $x$ and $x_{0}$ were defined as

$$
\begin{aligned}
x & \equiv P_{\mathrm{s}}\left(\frac{G \widetilde{M} \rho_{\mathrm{s}}}{4 R_{\mathrm{s}}}\right)^{-1} \propto\left(1+U_{\mathrm{s}} \sqrt{\frac{R_{\mathrm{s}}}{G \widetilde{M}}}\right)^{2}, \\
x_{0} & \equiv 3(\Gamma-1) \frac{\Delta E_{\mathrm{g}}}{4 \pi R_{\mathrm{s}}^{3}}\left(\frac{G \widetilde{M} \rho_{\mathrm{s}}}{4 R_{\mathrm{s}}}\right)^{-1} \propto-\frac{\Delta E_{\mathrm{g}}}{\dot{M} \sqrt{R_{\mathrm{s}} \widetilde{M}}}
\end{aligned}
$$

The proportionality relations can be verified by using Eqs. (39), (43) (with $\alpha=1 / \sqrt{2}$ ) and (44).

Equation (113) is the key equation to understand the behavior of the supernova shock under the influence of accretion and neutrino heating. Typically, $\Delta E_{\mathrm{g}}<0$ during the shock stagnation phase, and therefore $x_{0}<0$. Equation (113) depends on two variables which constrain the conditions at the shock front, namely on $x>0$ and on $y \equiv R_{\mathrm{s}} / R_{\mathrm{g}}$, for which $y \geq 1$ holds. Fixing the parameters $\dot{M}, \widetilde{M}$ and $R_{\mathrm{s}}$, one can show that a larger value of $x$ and thus a larger $U_{\mathrm{s}}$ requires that $x_{0}$ and therefore $\Delta E_{\mathrm{g}}$ is bigger (i.e., less negative). Physically, this corresponds to the case where neutrino energy deposition leads to a rising postshock pressure $P_{\mathrm{s}}$ (compare Eq. (114)), which accelerates the shock front. On the other hand, if $\left|U_{\mathrm{s}}\right| \ll\left|v_{\mathrm{p}}\right| \approx\left(G \widetilde{M} / R_{\mathrm{s}}\right)^{1 / 2}$ the quantity $x$ is essentially constant, and $y \propto R_{\mathrm{s}}^{7 / 16}$ (cf. Eq. (99)) is the variable which reacts to changes of $x_{0}$. The corresponding discussion is more transparent when Eq. (113) is rewritten in the following form:

$\left[x-x_{0}+4(3 \Gamma-4)\right] x^{3} y^{3} \approx(x+y-1)^{4}+4(3 \Gamma-4) x^{3} y^{4}$.

For $x_{0}<0$ this equation has a solution $\hat{y}$ which shrinks, if $\Delta E_{\mathrm{g}}$ and thus $x_{0}$ is larger (i.e., less negative). Therefore the radius $R_{\mathrm{S}}$ of the shock, which is compatible with the assumptions, is smaller. Inversely, if $\Delta E_{\mathrm{g}}$ and $x_{0}$ are lower 
(more negative), $R_{\mathrm{s}}$ will be larger. This behavior can be explained by the observation that $\Delta E_{\mathrm{g}}$ decreases when matter with negative specific energy is accumulated in the gain region. Such an accumulation of mass will cause a growth of the shock radius. It should be noted that for $x$ of order unity (i.e., $\left|U_{\mathrm{s}}\right| \ll\left|v_{\mathrm{p}}\right|$ ) a solution $\hat{y} \geq 1$ of Eq. (116) exists only in case of $x_{0} \leq 0$. A positive value of $\Delta E_{\mathrm{g}}$, on the other hand, is compatible only with a sufficiently large shock velocity $U_{\mathrm{s}}$.

The situation is graphically illustrated in Fig. 3, where $\Delta M_{\mathrm{g}}$ from Eq. (97) and $\Delta E_{\mathrm{g}}$ from Eqs. (104) and (105) are plotted as functions of $U_{\mathrm{s}}$ for different choices of the shock radius $R_{\mathrm{s}}$ (with parameters: $\widetilde{M}=1.25 M_{\odot}, \dot{M}=$ $0.3 M_{\odot} \mathrm{s}^{-1}, \Gamma=\gamma=4 / 3, \beta=7, \alpha=1 / \sqrt{2}, g_{\mathrm{r}}=6.41$, $f_{\mathrm{r}}=1.16$ ). Figure 3 (or Eq. (100)) shows that a growth of the mass $\Delta M_{\mathrm{g}}$ in the gain region will cause an increase of $R_{\mathrm{s}}$. This means that $\mathrm{d} R_{\mathrm{s}} / \mathrm{d} t \geq 0$ can be ensured if

$\frac{\mathrm{d}}{\mathrm{d} t}\left(\Delta M_{\mathrm{g}}\right) \geq 0$.

This, however, is not a sufficient criterion for a continued outward motion of the shock. The dotted arrows in Fig. 3 indicate a situation where the decrease of energy in the gain region is so large that the increase of the shock radius implies a deceleration of the shock. For moving along the path marked by solid arrows, i.e., for obtaining stable shock expansion with $\mathrm{d} U_{\mathrm{s}} / \mathrm{d} t \geq 0$, a necessary condition is

$$
\frac{\mathrm{d}}{\mathrm{d} t}\left(\Delta E_{\mathrm{g}}\right) \geq U_{\mathrm{s}}\left[\frac{\partial\left(\Delta E_{\mathrm{g}}\right)}{\partial R_{\mathrm{s}}}\right]_{U_{\mathrm{s}}} \text {. }
$$

The right hand side of Eq. (118) cannot be zero in general, because $\mathrm{d}\left(\Delta E_{\mathrm{g}}\right) / \mathrm{d} t>0$ can also be associated with a shrinkage of $R_{\mathrm{s}}$ if more matter (with negative total energy) is lost from the gain region by advection through the gain radius than is resupplied by gas falling into the shock. The combined conditions of Eqs. (117) and (118) guarantee that $R_{\mathrm{S}}$ and $U_{\mathrm{s}}$ grow at the same time. Applied to a stalled shock, in which case $U_{\mathrm{s}}=0$, Eq. (117) together with Eq. (118) can therefore be considered as "shock revival criterion", which states that for an expansion and acceleration of the shock front to occur, the energy in the gain region should increase and simultaneously the mass in the gain region should not decrease.

\subsection{Shock revival criterion}

If the conditions between neutrinosphere and shock vary slowly with time, $\dot{R}_{\mathrm{g}} \approx 0$ is a good assumption. Since $v_{\mathrm{p}} \neq 0$, Eq. (109) can then be written in the form

$$
\frac{\mathrm{d}}{\mathrm{d} t}\left(\Delta E_{\mathrm{g}}\right) \approx-\dot{M} l_{\mathrm{s}}+\dot{M}\left(l_{\mathrm{s}}-\frac{P_{\mathrm{s}}}{\rho_{\mathrm{p}}}\right) \frac{\dot{R}_{\mathrm{s}}}{v_{\mathrm{p}}}+\dot{M}^{\prime} l_{\mathrm{g}}+\mathcal{H}-\mathcal{C} \text {. }
$$

Replacing $l_{\mathrm{s}}$ in the bracket on the right hand side of Eq. (119) by Eq. (110) and using Eq. (39) for $P_{\mathrm{s}} / \rho_{\mathrm{p}}$, one derives in case of $\left|U_{\mathrm{s}}\right| \ll\left|v_{\mathrm{p}}\right|$ the expression

$$
\begin{aligned}
\frac{\mathrm{d}}{\mathrm{d} t}\left(\Delta E_{\mathrm{g}}\right) \approx & -\dot{M} l_{\mathrm{s}}-\dot{M}\left(2-\frac{1}{\beta}-\frac{\beta-1}{\beta^{2}} \frac{\Gamma}{\Gamma-1}\right) v_{\mathrm{p}} \dot{R}_{\mathrm{s}} \\
& +\dot{M}^{\prime} l_{\mathrm{g}}+\mathcal{H}-\mathcal{C} .
\end{aligned}
$$

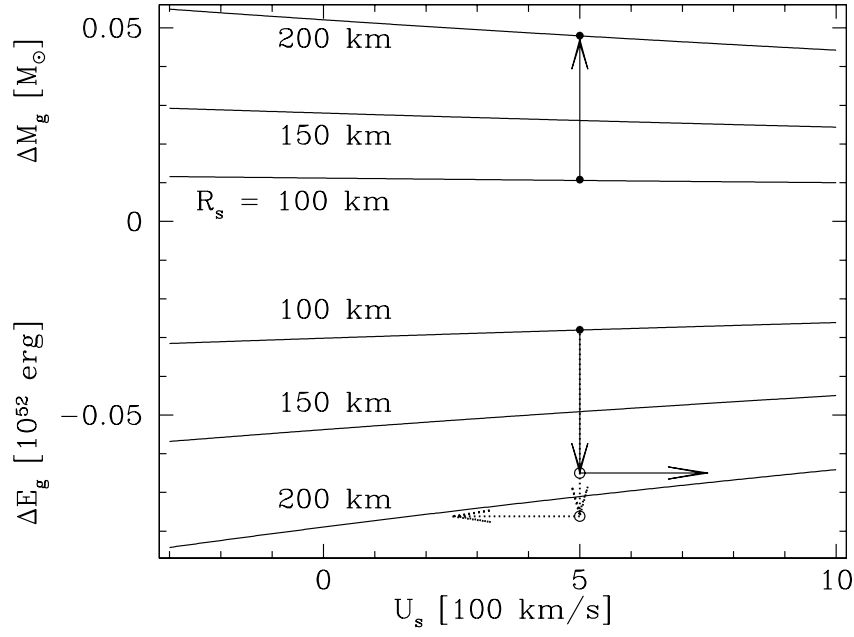

Fig. 3. Mass $\Delta M_{\mathrm{g}}$ and energy $\Delta E_{\mathrm{g}}$ in the gain region as functions of shock velocity $U_{\mathrm{s}}$ for different shock radii $R_{\mathrm{s}}$. The values of neutron star mass $\widetilde{M}$ and mass infall rate $\dot{M}$ into the shock are fixed. Two possible cases for a transition from initial shock radius $R_{\mathrm{s}}=100 \mathrm{~km}$ to final shock radius $R_{\mathrm{s}}=200 \mathrm{~km}$ are indicated. The path marked by solid arrows corresponds to stable shock expansion, the situation marked by dotted arrows means a slow-down of the shock

This equation is correct to first order in $\left|\dot{R}_{\mathrm{s}} / v_{\mathrm{p}}\right| \ll 1$. From the discussion in Sect. 8.2 follows that for an outward acceleration of a stalled shock $\left(\dot{R}_{\mathrm{s}}=U_{\mathrm{s}}=0\right)$, a necessary condition is (see Eq. (118)):

$\frac{\mathrm{d}}{\mathrm{d} t}\left(\Delta E_{\mathrm{g}}\right)=-\dot{M} l_{\mathrm{s}}+\dot{M}^{\prime} l_{\mathrm{g}}+\mathcal{H}-\mathcal{C}>0$.

Note that neutrino heating $(\mathcal{H})$ and cooling $(\mathcal{C})$ in the gain region as well as the mass accretion rate $\dot{M}$ through the shock have a direct influence. But also neutrino losses below $R_{\mathrm{g}}$ have an effect by determining $\dot{M}^{\prime}$, and a more indirect one by causing additional energy deposition in the gain region, where the neutrino energy extracted from the cooling layer is partly reabsorbed.

The terms proportional to $\dot{M}=4 \pi R_{\mathrm{s}}^{2} \rho_{\mathrm{p}} v_{\mathrm{p}}$ account for the so-called ram pressure of the infalling matter, which is proportional to $\rho_{\mathrm{p}} v_{\mathrm{p}}^{2}$ and damps shock expansion, because the accretion of matter through the shock yields a negative contribution to the right hand sides of Eqs. (119)-(121). A comparison of Eqs. (120) and (121) shows that the onset of shock expansion enhances this and therefore Eq. (121) gives a minimum requirement.

Instead of just an outward acceleration of the shock, a positive postshock velocity, i.e., $v_{\mathrm{s}}>0$, may be considered as a stronger criterion for the possibility of an explosion. With $\beta=\rho_{\mathrm{s}} / \rho_{\mathrm{p}}$ and Eq. (36) one derives $v_{\mathrm{s}}=\beta^{-1}\left(v_{\mathrm{p}}-\right.$ $\left.U_{\mathrm{s}}\right)+U_{\mathrm{s}}$, which means that $v_{\mathrm{s}}>0$ translates into $U_{\mathrm{s}}>$ $-v_{\mathrm{p}} /(\beta-1)$. Since $\beta \gg 1$ (Eq. (41)), this condition is fulfilled while $\left|U_{\mathrm{s}} / v_{\mathrm{p}}\right| \ll 1$ still holds. Using this more rigorous criterion will therefore affect the details of the discussion, but will not change the picture qualitatively.

$\mathrm{d}\left(\Delta E_{\mathrm{g}}\right) / \mathrm{d} t>0$ can be achieved by strong neutrino heating $\left(\mathcal{H}\right.$ large), but can also result if $\dot{M}^{\prime} l_{\mathrm{g}}>\dot{M} l_{\mathrm{s}}$. 
For $l_{\mathrm{g}}=l_{\mathrm{s}}$, which is true when $\Gamma=\gamma$ (see Eq. (112)), this is equivalent to $\dot{M}^{\prime}<\dot{M}$, i.e., when less mass is accreted through the shock than is lost from the gain region into the neutron star (note that $l_{\mathrm{s}}<0$; Eq. (110)). As a consequence, however, the mass between $R_{\mathrm{g}}$ and $R_{\mathrm{s}}$ and therefore the shock radius will decrease, in conflict with the demand for shock expansion (see Sect. 9.1). To make sure the shock expands, also Eq. (117) has to be fulfilled. In case of $U_{\mathrm{s}}=0, \dot{R}_{\mathrm{g}}=0$, Eq. (102) yields:

$\frac{\mathrm{d}}{\mathrm{d} t}\left(\Delta M_{\mathrm{g}}\right)=-\dot{M}+\dot{M}^{\prime} \geq 0 \Longleftrightarrow \dot{M} \leq \dot{M}^{\prime}$

Both Eqs. (121) and (122) constrain the parameters for which a revival of the stalled shock can occur.

\subsection{Conditions for shock revival}

The properties of Eq. (121) together with Eq. (122) will now be discussed in more detail. For chosen fixed values of the shock stagnation radius, those combinations of mass accretion rate $\dot{M}$ and neutrinospheric luminosity $L_{\nu}$ will be determined which allow for an outward acceleration of the shock front. For these conditions an explosion driven by neutrino energy deposition may develop.

Assuming $U_{\mathrm{s}}=0$ the gain radius is given by Eq. (99). For the neutrino luminosity $L_{\nu}=2 L_{\nu_{\mathrm{e}}}$ will be taken again. The accretion rate $\dot{M}^{\prime}$ of Eq. (93) can be calculated by using Eqs. (94) and (95). Neutrino effects are evaluated from Eqs. (74)-(77) and Eqs. (83) and (87) with Eq. (88) for the postshock density $\rho_{\mathrm{s}}$.

Several consistency constraints have to be taken into account to make sure that the assumptions of the analytic model developed in the preceding sections are fulfilled:

(i) For the gain radius (Eq. (99)) $R_{\nu}+h \lesssim R_{\mathrm{g}} \leq R_{\mathrm{S}}$ must hold. Here $h$ is the scale height of the exponential neutron star atmosphere, Eq. (51). The left inequality constrains the neutrinospheric luminosity to be $L_{\nu} \lesssim L_{1}(\dot{M})$, where the limit $L_{1}$ depends on the accretion rate $\dot{M}$. The right inequality, on the other hand, requires $L_{\nu} \geq L_{2}(\dot{M})$;

(ii) Since the neutrinospheric luminosity $L_{\nu}$ and temperature $T_{\nu}$ are not coupled here by the assumption of blackbody emission, Eq. (78) must be satisfied to have $L_{\text {acc }} \geq 0$, i.e., to have a cooling layer outside of the neutrinosphere. This translates into a condition $L_{\nu} \leq L_{3}$;

(iii) The definition of $R_{\mathrm{g}}$ implies that neutrinos transfer energy to the stellar gas for $R_{\mathrm{g}} \leq r \leq R_{\mathrm{s}}$. Therefore Eq. (87) has to fulfill the condition $\mathcal{H}-\mathcal{C} \geq 0$, corresponding to $L_{\nu} \geq L_{4}(\dot{M})$. This constraint is similar to the one which follows from the requirement that $R_{\mathrm{g}} \leq R_{\mathrm{s}}$, but somewhat stronger, depending on the value of the ratio between $\left\langle\mu_{\nu}\right\rangle_{\mathrm{g}}$ and $\left\langle\mu_{\nu}\right\rangle^{*}$;

(iv) Equation (89) (with $\mathcal{H}$ taken from Eq. (83)) has to be less than about 0.5 to justify the disregard of reabsorption of neutrinos emitted from the gain layer. This limits the neutrinospheric luminosity to $L_{\nu} \lesssim L_{5}(\dot{M})$; (v) The postshock temperature must be $k T_{\mathrm{s}} \gtrsim 1 \mathrm{MeV}$ because the matter behind the shock is assumed to be completely disintegrated into free nucleons, and $\alpha$ particles therefore do not exist. For this to hold, the absolute value of the mass accretion rate must exceed some lower limit, $|\dot{M}| \gtrsim\left|\dot{M}_{1}\right|$, where $\dot{M}_{1}$ depends on the shock radius and the effective mass $\widetilde{M}$ of the remnant;

(vi) Since self-gravity of the gas between neutrinosphere and shock was neglected, the total mass there must be much smaller than the mass of the neutron star. This requirement leads to an upper limit for the rate of mass accretion: $|\dot{M}| \lesssim\left|\dot{M}_{2}\right|$.

While conditions (i)-(iii) ensure the logical coherence of the model, a violation of conditions (iv)-(vi) would just reduce the accuracy of the discussion. For example, the framework developed in the previous sections can be generalized such that the (partial) recombination of nucleons to $\alpha$ particles or heavy nuclei at temperatures below about one $\mathrm{MeV}$ is taken into account. Item (vi) implies

$$
\begin{aligned}
\int_{R_{\nu}}^{R_{\mathrm{s}}} \mathrm{d} r 4 \pi r^{2} \rho(r) & =\int_{R_{\nu}}^{R_{\mathrm{eos}}} \mathrm{d} r 4 \pi r^{2} \rho(r)+\int_{R_{\mathrm{eos}}}^{R_{\mathrm{s}}} \mathrm{d} r 4 \pi r^{2} \rho(r) \\
& \lesssim \int_{R_{\nu}}^{\infty} \mathrm{d} r 4 \pi r^{2} \rho_{1}(r)+\int_{R_{\nu}+h}^{R_{\mathrm{s}}} \mathrm{d} r 4 \pi r^{2} \rho_{2}(r),
\end{aligned}
$$

where $\rho_{1}(r) \equiv \rho_{\nu} \exp \left[-\left(r-R_{\nu}\right) / h\right]$ (Eq. (50) with $h$ from Eq. (51)) and $\rho_{2}(r) \equiv \rho_{\mathrm{s}}\left(R_{\mathrm{s}} / r\right)^{3}$ (Eq. (63)). A reasonable upper bound for this mass integral is

$$
\int_{R_{\nu}}^{R_{\mathrm{s}}} \mathrm{d} r 4 \pi r^{2} \rho(r) \lesssim \frac{\widetilde{M}}{5},
$$

which limits the allowed accretion rate according to condition (vi).

The sequence of plots in Fig. 4 shows the results of an evaluation of Eqs. (121) and (122) together with the constraints (i)-(vi) for different shock stagnation radii: $R_{\mathrm{s}}=100,150,200,250$ and $300 \mathrm{~km}$, respectively. The numerical values chosen for the other parameters were: $\widetilde{M}=1.25 M_{\odot}, R_{\nu}=50 \mathrm{~km}, k T_{\nu}=4 \mathrm{MeV}, \beta=7$, $\Gamma=\gamma=\frac{4}{3}, f_{\mathrm{g}}=1.25$ (corresponding to $\eta_{\mathrm{e}} Y_{\mathrm{e}} \approx 1$ at the neutrinosphere), $q_{\mathrm{d}}=8.510^{18} \mathrm{erg} \mathrm{g}^{-1},\left\langle\mu_{\nu}\right\rangle^{*}=0.7$, $\left\langle\mu_{\nu}\right\rangle_{\mathrm{g}}=0.6$, and $\widehat{\left\langle\mu_{\nu}\right\rangle}=0.4$.

The roots of $\mathrm{d}\left(\Delta E_{\mathrm{g}}\right) / \mathrm{d} t$ and $\mathrm{d}\left(\Delta M_{\mathrm{g}}\right) / \mathrm{d} t$ are represented by the lines labeled with $\mathrm{O}_{\mathrm{E}}$ and $\mathrm{O}_{\mathrm{M}}$, respectively. These lines separate regions in the $|\dot{M}|-L_{\nu}$ plane, within which the collapsed stellar core reacts differently to the mass inflow through the shock and to the irradiation by neutrinos emitted from the neutrinosphere (and from the cooling layer). In this respect the plots of Fig. 4 can be considered as "phase diagrams" for the postbounce evolution of the supernova. Within the hatched areas both Eqs. (121) and (122) are simultaneously fulfilled. Additional lines correspond to constraints (i)-(vi). 

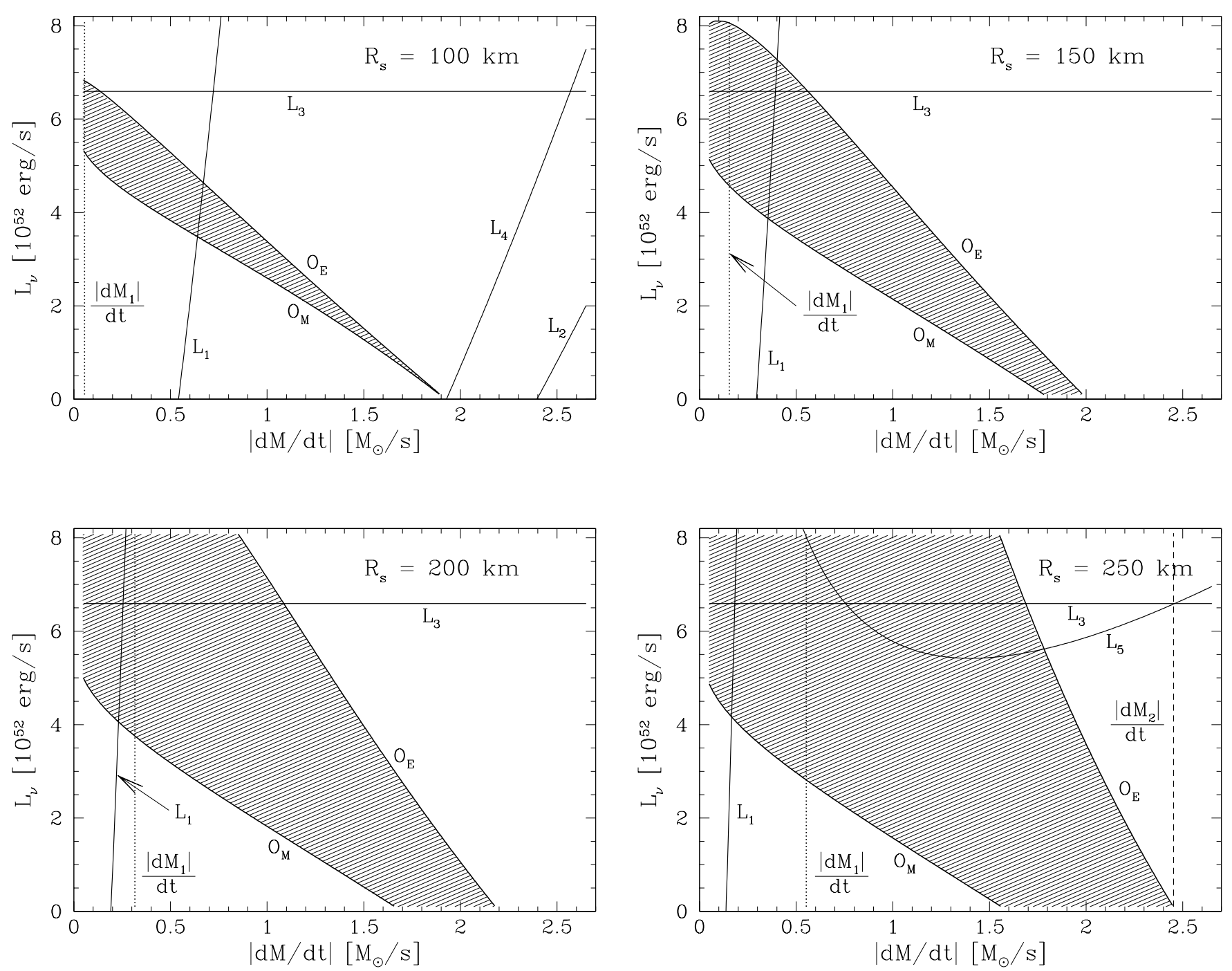

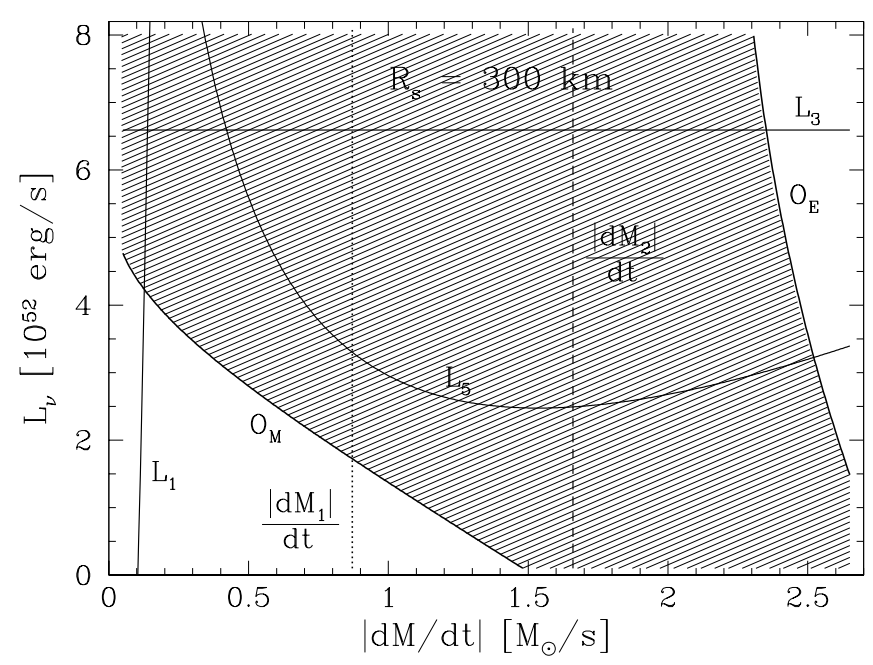

They are displayed as warning flags that the assumptions of the treatment may need to be generalized. Left of the vertical dotted line, which corresponds to constraint (v), $\alpha$
Fig. 4. Conditions for shock revival by neutrino heating for different shock stagnation radii $R_{\mathrm{s}}$. The lines labeled with $\mathrm{O}_{\mathrm{E}}$ and $\mathrm{O}_{\mathrm{M}}$ connect the roots of $\mathrm{d}\left(\Delta E_{\mathrm{g}}\right) / \mathrm{d} t$ and $\mathrm{d}\left(\Delta M_{\mathrm{g}}\right) / \mathrm{d} t$, respectively, in the plane defined by the mass accretion rate into the shock, $\dot{M}$, and the neutrinospheric luminosity $L_{\nu}$. The curves with labels $L_{i}(i \in\{1, \ldots, 5\})$ and $\left|\mathrm{d} M_{j} / \mathrm{d} t\right|(j \in\{1,2\})$ correspond to the constraints (i)-(vi) listed in Sect. 9.3. They represent warning flags indicating that the assumptions of the discussion may need to be generalized. The hatched areas mark the regions where the conditions are favorable for a neutrino-driven explosion, because Eqs. (121) and (122) are both satisfied such that the supernova shock expands and accelerates. Below the curve $\mathrm{O}_{\mathrm{M}}$ the rate of mass loss from the gain layer to the neutron star exceeds the mass accretion rate $\dot{M}$ and therefore $\mathrm{d}\left(\Delta M_{\mathrm{g}}\right) / \mathrm{d} t$ is negative. Above the curve $\mathrm{O}_{\mathrm{E}}$ the energy deposition by neutrino heating cannot compensate for the accumulation of mass with negative total energy in the gain region and therefore $\mathrm{d}\left(\Delta E_{\mathrm{g}}\right) / \mathrm{d} t$ is negative

particles and heavy nuclei in the postshock medium would have to be taken into account, and the analysis performed here is not very accurate. The vertical dashed line marks 

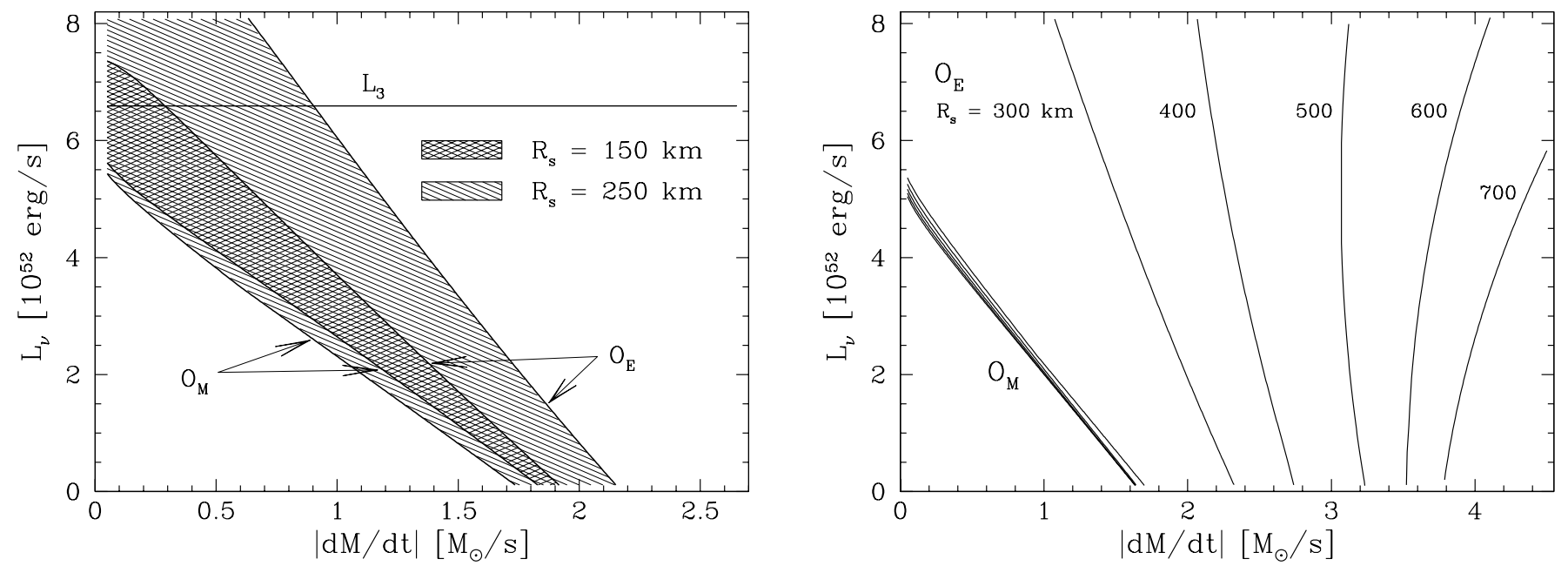

Fig. 5. Left: conditions for shock revival by neutrino heating for shock stagnation radii $R_{\mathrm{s}}=150 \mathrm{~km}$ (cross-hatched area) and $250 \mathrm{~km}$ (hatched area). Right: the lines $\mathrm{O}_{\mathrm{M}}$ and $\mathrm{O}_{\mathrm{E}}$ which connect the roots of Eqs. (121) and (122), respectively, for shock radii $300 \mathrm{~km}, 400 \mathrm{~km}, 500 \mathrm{~km}, 600 \mathrm{~km}$, and $700 \mathrm{~km}$. Different from Fig. 4, the gain radius and the integrals for neutrino heating and cooling in the gain layer were evaluated by using the exact solution for hydrostatic equilibrium instead of approximate power-law profiles. In addition, the disappearance of free nucleons and therefore the quenching of neutrino absorption and emission below a temperature of $1 \mathrm{MeV}$ were taken into account. Above the line labeled with $L_{3}$ the accretion luminosity $L_{\text {acc }}($ Eq. (77)) becomes negative. The lines corresponding to constraints (v) and (vi) are omitted for reasons of clarity

the boundary right of which Eq. (124) and thus constraint (vi) is violated.

Two of the simplifications that entered the analysis for Fig. 4 can be easily removed. On the one hand, the gain radius $R_{\mathrm{g}}$ and heating and cooling in the gain layer can be calculated more accurately, when the density and temperature profiles of Eqs. (59) and (61) instead of the power-law approximation of the hydrostatic atmosphere [Eq. (63)] are used. In this case $R_{\mathrm{g}}$ must be numerically determined as the root of Eq. (33) (with $L_{\nu_{\mathrm{e}}}=\frac{1}{2} L_{\nu}\left(R_{\mathrm{g}}\right)$ as given by Eq. (76)), and the integrals for $\mathcal{H}$ and $\mathcal{C}$ in Eq. (82) can also be evaluated numerically. On the other hand, the recombination of free nucleons to $\alpha$ particles and heavy nuclei at low temperatures can roughly be taken into account concerning its effects on the neutrino interaction in the gain region. Provided that above a certain temperature, say $1 \mathrm{MeV}$, all nuclei are disintegrated into free nucleons and below this temperature all nucleons are bound in nuclei, neutrino absorption and emission reactions will not take place outside of the corresponding radius $R_{\alpha}$. The latter can also be calculated from the temperature profile of Eq. (61). The heating and cooling integrals are then performed with the upper integration boundary being chosen as the minimum of $R_{\mathrm{s}}$ and $R_{\alpha}$. The recombination of nucleons to $\alpha$ particles releases a sizable amount of energy, about $7 \mathrm{MeV}$ per nucleon. This additional energy source in the gain region was not included in the discussion here, because it requires a detailed modelling of the composition history of the postshock medium (considering the different degree of disintegration of nuclei during infall and recombination of nucleons during later expansion in different volumes of matter).

The results of this more general treatment are displayed in Fig. 5 for shock radii $R_{\mathrm{s}}=150 \mathrm{~km}$ and $250 \mathrm{~km}$.
The quantitative changes are significant: Compared to Fig. 4 the different value for the $\mathcal{H}-\mathcal{C}$ term moves the $\mathrm{O}_{\mathrm{M}}$-line slightly upward and the $\mathrm{O}_{\mathrm{E}}$-line more strongly downward. A similar effect is associated with a moderate reduction of the shock radius in Fig. 4. The gain radius obtained by the exact calculation can also shrink with growing shock radius, different from the approximate representation of Eq. (99). On the other hand, the outer boundary of the gain layer is defined by the recombination radius $R_{\alpha}$ of $\alpha$ particles instead of the possibly larger shock radius. Both effects combined, the total heating rate in the gain layer is similar. Therefore the qualitative picture remains unchanged.

The hatched areas in the plots of Figs. 4 and 5 include those combinations of parameters for which the conditions of Eqs. (121) and (122) are both satisfied and therefore the initially stagnant shock will expand and will be accelerated. Below the $\mathrm{O}_{\mathrm{M}}$ line neutrino cooling outside of the neutrinosphere is very efficient and the neutron star swallows matter faster than gas is resupplied by accretion through the shock. Therefore $\mathrm{d}\left(\Delta M_{\mathrm{g}}\right) / \mathrm{d} t$ is negative. When the mass accretion rate $|\dot{M}|$ drops below this critical line, shock expansion can be supported only by an increasing core luminosity, because a larger value of $L_{\nu}$ reduces the neutrino losses from the cooling region. Otherwise advection through the gain radius and thus into the neutron star extracts mass from the neutrino-heating region and the shock retreats. Figures 4 and 5 show that for given rate $|\dot{M}|$ there is a lower limit of the neutrinospheric luminosity $L_{\nu}$, which must be exceeded when shock expansion and acceleration shall occur.

Above the $\mathrm{O}_{\mathrm{E}}$ line neutrino heating (represented by the $\mathcal{H}-\mathcal{C}$ term in Eq. (121)) cannot compete with the accumulation of matter with negative total energy in the 
gain layer. In this case $\mathrm{d}\left(\Delta E_{\mathrm{g}}\right) / \mathrm{d} t$ is negative. $R_{\mathrm{s}}$ can nevertheless grow for such conditions, simply because gas piles up on top of the neutron star. This pushes the shock farther out, but does not allow positive postshock velocities to develop. Since the postshock matter is gravitationally bound $\left(\Delta E_{\mathrm{g}}<0\right)$, an explosion, however, requires sufficiently powerful energy input by neutrinos. The position of the $\mathrm{O}_{\mathrm{E}}$ line shifts with changing shock radius. For discussing the destiny of the shock this change of the overall situation associated with the shock motion therefore has to be taken into account. This can be done by solving the equations of the toy model for time-dependent information about the shock radius and the shock velocity (see Sect. 9.4).

The $\mathrm{O}_{\mathrm{E}}$ line is very sensitive to the shock position, whereas the $\mathrm{O}_{\mathrm{M}}$ line is only weakly dependent (Fig. 5). On the one hand, a high core luminosity $L_{\nu}$ reduces the downward advection of gas through the gain radius. On the other hand, the neutrino heating in the gain layer increases with larger shock radius. Both effects determine the slopes and positions of the critical lines. The distance between the $\mathrm{O}_{\mathrm{M}}$ and $\mathrm{O}_{\mathrm{E}}$ lines in Figs. 4 and 5 grows for larger shock radii, and the hatched area expands. This is caused by an increase of the $\mathcal{H}-\mathcal{C}$ term in Eq. (121).

Acceleration is easier for a shock which has stalled at a large distance from the center, i.e., the same core luminosity can then ensure favorable conditions already for a higher value of $|\dot{M}|$. Besides stronger neutrino heating in the more extended gain region, another effect contributes to this. The increase of the postshock pressure which is necessary to accelerate the standing shock to a positive velocity $U_{\mathrm{s}} \ll\left|v_{\mathrm{p}}\right|$ is given by

$$
\begin{aligned}
\Delta P_{\mathrm{s}} & =P_{\mathrm{s}}\left(U_{\mathrm{s}}\right)-P_{\mathrm{s}}(0) \\
& \cong-2\left(1-\frac{1}{\beta}\right) \rho_{\mathrm{p}} v_{\mathrm{p}} U_{\mathrm{s}}=-\left(1-\frac{1}{\beta}\right) \frac{\dot{M} U_{\mathrm{s}}}{2 \pi R_{\mathrm{s}}^{2}} .
\end{aligned}
$$

This pressure increase is lower when the shock radius $R_{\mathrm{s}}$ is big.

The $\mathrm{O}_{\mathrm{M}}$ line defines a critical curve for the shock evolution, whose slope and position are hardly dependent on the shock radius. It can be approximated analytically by solving Eq. (122) in case of $\dot{M}^{\prime}=\dot{M}$ for the critical core luminosity $L_{\nu}^{*}$ as a function of $\dot{M}$. One derives

$L_{\nu}^{*}(\dot{M}) \approx \frac{\mathrm{e}^{-a} b(1-0.1 \omega)-\dot{M}\left(l_{\nu}+q_{\mathrm{d}}\right)}{1-\mathrm{e}^{-a}(1-0.1 \omega)}$,

where $l_{\nu}$ is defined as the quantity $l=(e+P) / \rho-G \widetilde{M} / r$ at the neutrinosphere (cf. Eqs. (93) and (95)), $a$ and $b$ were introduced in Eqs. (74) and (75), respectively, and $\omega$ is given by

$\omega \equiv \frac{\left(k T_{\nu_{\mathrm{e}}, 4}\right)^{2}}{\left\langle\mu_{\nu}\right\rangle^{*}} \frac{(-\dot{M})}{M_{\odot} / \mathrm{s}}\left(\frac{\widetilde{M}}{M_{\odot}}\right)^{-\frac{1}{2}} R_{\mathrm{s}, 7}^{-1 / 2}\left[\left(\frac{R_{\mathrm{s}}}{R_{\mathrm{g}}}\right)^{2}-1\right]$.

To obtain Eq. (126) use was made of Eqs. (66), (76), (77), (83), (87), (88), (93), (94), and (99). The term in the curly brackets of Eq. (87) was assumed to be equal to $\frac{2}{3}$, and the rather weak dependence of the gain radius in Eq. (127) on the neutrino luminosity was ignored in writing $L_{\nu}^{*}$ in the explicit form of Eq. (126). In the latter equation $\omega$ also depends on the mass infall rate $\dot{M}$. For representative shock radii and accretion rates, $\omega$ is found to be of order unity: $\omega \sim 1$. With this, $L_{\nu}^{*}$ becomes a simple linear function of $\dot{M}$. Inserting the parameter values used for Figs. 4 and 5 (listed after Eq. (124)), one ends up with

$L_{\nu}^{*}(\dot{M}) \approx\left(5.6-3.3 \frac{(-\dot{M})}{M_{\odot} / \mathrm{s}}\right) 10^{52} \frac{\mathrm{erg}}{\mathrm{s}}$

This expression has a root for $\dot{M}=-1.7 M_{\odot} \mathrm{s}^{-1}$ and fits the $\mathrm{O}_{\mathrm{M}}$ lines in Figs. 4 and 5 reasonably well.

\subsection{Time-dependent solutions}

The equations of the toy model developed in this paper can be solved for the shock radius $R_{\mathrm{s}}(t)$ and the shock velocity $U_{\mathrm{s}}(t)$ as functions of time. For this purpose the mass and energy in the gain layer have to be evolved according to the conservation laws of Eqs. (102) and (109). Together with $U_{\mathrm{s}}=\mathrm{d} R_{\mathrm{s}} / \mathrm{d} t \equiv \Delta R_{\mathrm{s}} / \Delta t$ these equations were integrated implicitly in time, with the velocity of the gain radius given by $\dot{R}_{\mathrm{g}} \equiv \Delta R_{\mathrm{g}} / \Delta t$ for time step $\Delta t$. The mass in the gain layer, $\Delta M_{\mathrm{g}}$, and the corresponding total (internal plus gravitational) energy, $\Delta E_{\mathrm{g}}$, were initially calculated from Eq. (97) and Eqs. (104) and (105), respectively. The gain radius $R_{\mathrm{g}}$ and the heating and cooling integrals for the gain layer were evaluated using the exact solution of hydrostatic equilibrium (Eqs. (59)-(61)) with the option to chose an arbitrary value for the structural polytropic index $\gamma$ (Eq. (62)). The quenching of neutrino absorption and emission reactions by the recombination of free nucleons to $\alpha$ particles and heavy nuclei below a temperature around $1 \mathrm{MeV}$ was taken into account.

The postshock density is related to the preshock density by $\rho_{\mathrm{s}}=\beta \rho_{\mathrm{p}}$, and the postshock pressure is given by Eq. (39). The density contrast $\beta$ as well as the pressure jump at the shock are affected by the conditions in the gain layer. The latter is assumed to be in hydrostatic equilibrium with mass inflow from the infall region and additional gain or loss by mass exchange with the neutron star. Therefore simultaneous conservation of mass and energy requires that $\beta$ is allowed to float, just as $P_{\mathrm{s}}$ is a degree of freedom which adjusts in response to the energy input due to the heating by neutrinos. This means that $\beta$ is also considered as a variable which the set of equations is solved for.

Although generalization is straightforward, the neutron star mass $\widetilde{M}$, the mass accretion rate into the shock, $\dot{M}$, and the neutrinospheric parameters $\left(L_{\nu}, T_{\nu}, R_{\nu}\right)$ were kept constant with time for reasons of simplicity. Supernova calculations show that during a transient, but rather short period of several $10 \mathrm{~ms}$ up to about $100 \mathrm{~ms}$ after bounce, $L_{\nu}$ and $\dot{M}$ decrease from very high values to a much lower level, and lateron change only slowly 

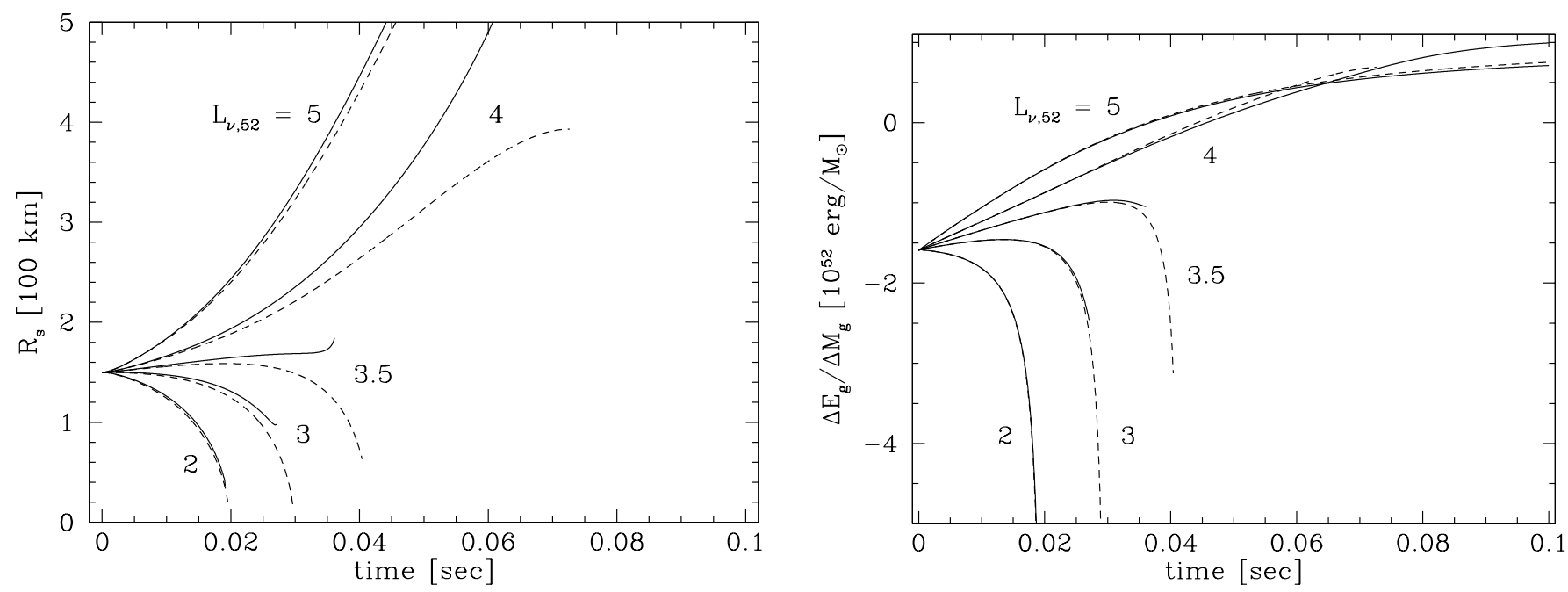

Fig. 6. Shock radius (left) and specific energy in the gain layer (right) as functions of time for different neutrinospheric luminosities (measured in units of $10^{52} \mathrm{erg} \mathrm{s}^{-1}$ ) and for an initial shock stagnation radius of $150 \mathrm{~km}$ [with $U_{\mathrm{s}}(t=0)=0$ ]. The structural polytropic index of the gain layer was chosen to be $\gamma=4 / 3$, the mass accretion rate into the shock $|M|=0.3 M_{\odot} \mathrm{s}^{-1}$, the neutron star mass $\widetilde{M}=1.25 M_{\odot}$, and the neutrinospheric radius and temperature $R_{\nu}=50 \mathrm{~km}$ and $T_{\nu}=4 \mathrm{MeV}$, respectively. The gain radius and the integrals for neutrino heating and cooling in the gain layer were evaluated by using the exact solution for hydrostatic equilibrium. The disappearance of free nucleons and therefore the quenching of neutrino absorption and emission below a temperature of $1 \mathrm{MeV}$ were also taken into account. The dashed lines correspond to the case where the density jump in the shock was set to be large (see text)

with time (cf., for example, Fig. 2 in Rampp \& Janka 2000). A discussion of the subsequent destiny of the supernova shock should not be affected by this variation, because the shock expansion turns out to occur on a significantly shorter timescale (see below). The ongoing contraction of the neutron star and a corresponding change of the neutrinospheric temperature and luminosity, however, were found to have considerable influence (see Janka \& Müller 1996). For the exemplary purpose of the calculations reported on below, the introduction of additional, model-dependent degrees of freedom will nevertheless be abstained from.

\subsubsection{Results for different $L_{\nu}$ and fixed $\dot{M}$}

The shock radius $R_{\mathrm{S}}(t)$ and the specific energy in the gain layer, $\Delta E_{\mathrm{g}} / \Delta M_{\mathrm{g}}$, are shown as functions of time in Fig. 6 for different neutrinospheric luminosities $L_{\nu}$. The structural polytropic exponent $\gamma$ was set equal to the adiabatic index $\Gamma$ of the equation of state, both chosen to be $\frac{4}{3}$. The other parameters of the evaluation were $\widetilde{M}=1.25 M_{\odot}$, $R_{\nu}=50 \mathrm{~km}, T_{\nu}=4 \mathrm{MeV}$, and $|\dot{M}|=0.3 M_{\odot} \mathrm{s}^{-1}$. The initial shock radius was set to $150 \mathrm{~km}$ with $U_{\mathrm{s}}(t=0)=0$ and $\dot{R}_{\mathrm{g}}(t=0)=0$.

The solid lines display the case where $\beta$ was allowed to vary in all equations. Only for sufficiently high neutrinospheric luminosity the shock is able to expand to large radii. For lower $L_{\nu}$ the specific energy per nucleon in the gain layer begins to drop again at some stage of the evolution, and continued shock expansion is not possible, because the postshock pressure is not large enough for driving the shock out. The sudden positive acceleration of the shock front towards the end of the solid lines for these unsuccessful cases is a mathematical artifact, which occurs in response to the rapid decrease of the factor $\left(1-\beta^{-1}\right)$ in Eq. (39) as $\beta$ falls to unphysical values near unity. For a given value of $P_{\mathrm{s}}$, the decay of the term $\left(1-\beta^{-1}\right) \rightarrow 0$ is attempted to be compensated by a catastrophic increase of the factor $\left(v_{\mathrm{p}}-U_{\mathrm{s}}\right)^{2}$ in Eq. (39). In contrast to the solid lines, the dashed curves were obtained by explicity setting $\left(1-\beta^{-1}\right) \approx 1$ in Eq. (39). Thus assuming that the density jump in the shock is large, the shock velocity is solely determined by the value of the pressure $P_{\mathrm{s}}$ behind the shock. Therefore the dashed lines show the breakdown of the shock expansion more clearly than the solid lines. They confirm that shock recession is correlated with a decrease of the specific energy in the gain layer.

The properties of the time-dependent solutions for the shock radius agree with the discussion of Sects. 9.1-9.3. Keeping $\dot{M}$ fixed, there is a threshold value for the core luminosity above which the shock runs out to large radii and the energy per baryon in the gain layer becomes positive. A high neutrinospheric luminosity has two favorable effects: On the one hand the neutrino heating in the gain layer is larger, on the other hand the energy loss by neutrino emission in the cooling layer is lower, thus reducing the mass accretion into the neutron star and the mass loss from the gain layer. The case with $L_{\nu}=410^{52} \mathrm{erg} \mathrm{s}^{-1}$ is near the borderline between successful shock expansion and failure for the chosen set of parameters (compare also Fig. 5): The shock is already very weak when it has reached a radius of about $400 \mathrm{~km}$, which is clearly visible from the dashed lines. 

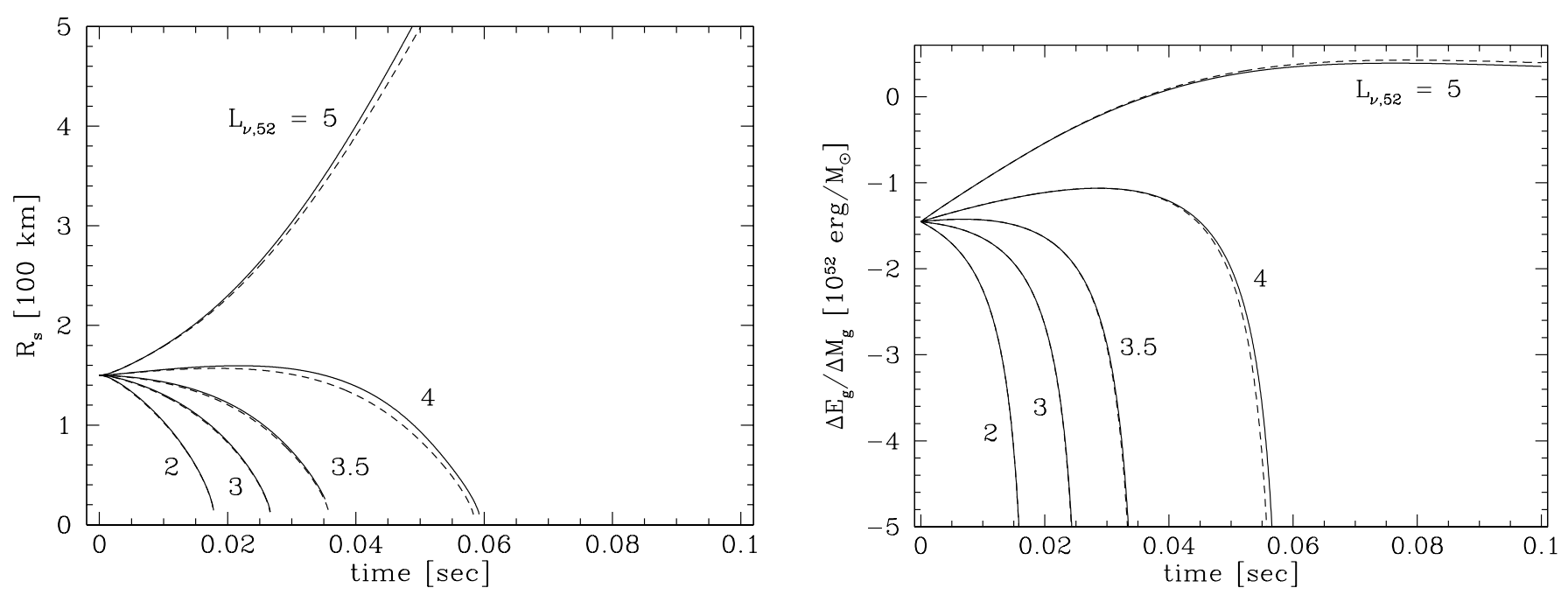

Fig. 7. Same as Fig. 6, but for a structural polytropic index $\gamma=1.45$ in the gain layer

The mass accretion rates, $\dot{M}^{\prime}$, of the nascent neutron star, which correspond to increasing values of the neutrinospheric luminosity $L_{\nu}$ in Fig. 6, are all negative, with values: $\left|\dot{M}^{\prime}\right|=1.15,0.84,0.68,0.53$, and 0.21 , and the $\nu_{\mathrm{e}}$ plus $\bar{\nu}_{\mathrm{e}}$ luminosities at the gain radius for these cases are: $L_{\nu}\left(R_{\mathrm{g}}\right)=5.0,5.3,5.5,5.7$, and $6.010^{52} \mathrm{erg} \mathrm{s}^{-1}$. Because of the contribution from the accretion luminosity, $L_{\nu}\left(R_{\mathrm{g}}\right)$ shows much less variation than the core luminosity $L_{\nu}$, and the neutrino heating in the gain layer is also similar. The accretion component is not dominant when the shock moves out. The breakdown of shock expansion is therefore associated with a low neutrinospheric luminosity which causes high mass loss from the gain layer, leading to a decrease of the pressure support behind the shock. At the same time the width of the gain layer shrinks, its optical depth drops, and the neutrino energy deposition decreases. This leads to a negative feedback and the shock recession accelerates dramatically.

The optical depth for $\nu_{\mathrm{e}}$ and $\bar{\nu}_{\mathrm{e}}$ absorption in the gain layer is given by Eq. (89), $\tau_{\mathrm{a}} \equiv \mathcal{H} / L_{\nu}\left(R_{\mathrm{g}}\right)$. Its value depends on the particular conditions, the shock position, mass infall rate into the shock, and the neutrinospheric luminosity, temperature and radius, which influence the position of the gain radius. For the models shown in Figs. 6 and 7 the initial value is between 0.15 and 0.18 . In case of shock expansion the optical depth increases for an intermediate period of time by up to 50 per cent due to the growth of the gain region. This improves the conditions for ongoing neutrino heating and leads to a rapid rise of the energy in the gain layer. The positive feedback also causes a sharp bifurcation in the behavior of cases of failing and successful shock expansion. Because neutrinos are not only absorbed, but also reemitted, the net effect of neutrino energy deposition is lowered somewhat. It scales with $(\mathcal{H}-\mathcal{C}) / L_{\nu}\left(R_{\mathrm{g}}\right)$, which has typically only about half the value of $\tau_{\mathrm{a}}$. When the gain layer expands, the temperature decreases and the reemission of neutrinos is reduced.

\subsubsection{Results for different $\dot{M}$}

When $|\dot{M}|$ lies below the $\mathrm{O}_{\mathrm{M}}$ line of Figs. 4 and 5, the shock expansion is suppressed. But also high mass infall rates damp the shock expansion, because a larger increase of the postshock pressure is needed for shock acceleration (see Eq. (125)) and the optical depth of the gain layer decreases (because the gain radius is farther out). For high $|\dot{M}|$ the shock therefore gains speed more slowly.

In case of very high mass infall rates $|\dot{M}|$ and small shock radii a gain layer does not exist. Provided the neutrino luminosity is sufficiently large such that $\dot{M}^{\prime}>\dot{M}$ (which is easily fulfilled for high $|\dot{M}|$ ), the shock is slowly pushed outward by the gas that stays in the layer between the neutron star and the shock. Eventually the postshock temperature will be low enough for a gain layer to form. With neutrino-heated gas accumulating above the gain radius (i.e., $\Delta M_{\mathrm{g}}$ increases) the shock moves even farther out, but the total energy in the gain layer decreases because the neutrino heating cannot compensate the negative binding energy of the growing gas mass. Only when the shock has reached a sufficiently large radius the situation becomes favorable for an explosion because then $\mathrm{d}\left(\Delta E_{\mathrm{g}}\right) / \mathrm{d} t>0$ (i.e., the conditions are now left of the corresponding $\mathrm{O}_{\mathrm{E}}$ line in Fig. 5). If this radius is very far out, because $|\dot{M}|$ is very high, the energy deposited in the gain layer may not be sufficient to produce a positive total energy in the gain layer. The gas behind the shock will stay bound and an explosion is not possible. The critical accretion rate for this to happen depends on the neutrinospheric parameters $\left(R_{\nu}, T_{\nu}\right.$ and $\left.L_{\nu}\right)$, to some degree also on the structural polytropic index $\gamma$. For the parameters and neutrino luminosities considered in the present discussion this value is found to be around $4 M_{\odot} \mathrm{s}^{-1}$.

Even for somewhat smaller absolute values of the accretion rate and a positive total energy in the gain layer explosions might not occur. The question, however, whether an outward running supernova shock will reach the 
stellar surface and what amount of matter it is able to eject, requires a global treatment of the problem, including the possible energy release by nuclear burning and recombination of nucleons, and including the energy which will be spent on lifting the stellar mantle and envelope in the gravitational field of the star. This is far beyond the limits of the current treatment, which focusses on a discussion of the conditions that are necessary for reviving a stalled shock and for pushing it out to a radius of $\gtrsim 1000 \mathrm{~km}$ by the neutrino heating mechanism.

\subsubsection{Thermodynamic conditions}

The entropy per nucleon in the gain layer, where relativistic electrons, positrons and photons as well as nonrelativistic nucleons and nuclei contribute to the pressure, is given by

$s=\frac{\varepsilon+P}{k T\left(\rho / m_{\mathrm{u}}\right)}-\sum_{i} \eta_{i} Y_{i}$,

where the sum runs over all kinds of particles $i$. When nuclei are fully dissociated, in which case $Y_{\mathrm{p}}=Y_{\mathrm{e}}$ and $Y_{\mathrm{n}}=1-Y_{\mathrm{e}}$, this sum can be written as

$$
\begin{aligned}
\sum_{i} \eta_{i} Y_{i}= & Y_{\mathrm{e}}\left(\eta_{\mathrm{e}}+\eta_{\mathrm{p}}-\eta_{\mathrm{n}}\right)+\eta_{\mathrm{n}} \\
\approx Y_{\mathrm{e}} \eta_{\mathrm{e}} & +Y_{\mathrm{e}} \ln \left(\frac{Y_{\mathrm{e}}}{1-Y_{\mathrm{e}}}\right) \\
& +\ln \left(1.2710^{-3} \frac{\left(1-Y_{\mathrm{e}}\right) \rho_{9}}{(k T)^{3 / 2}}\right) .
\end{aligned}
$$

Since typically $Y_{\mathrm{e}} \sim 0.2-0.3$ and $\eta_{\mathrm{e}} / \pi \lesssim 1$ around the gain radius, the combined terms scaling with $Y_{\mathrm{e}}$ are negligibly small. Using $P=(\Gamma-1) \varepsilon$, Eq. (129) can be evaluated for the models plotted in Figs. 6 and 7. Near the gain radius characteristic initial values of the entropy per nucleon are found to be between 10 and 14, with a contribution from relativistic degrees of freedom of $s_{\mathrm{r}} \sim 2-3$. This is in good agreement with results from detailed hydrodynamical models (see, e.g., Rampp \& Janka 2000). In case of successful shock expansion, the total entropy per nucleon increases to values between 25 and 30 towards the end of the computed evolution.

Clearly, neither the entropy nor the pressure are dominated by radiation and leptons, but baryons play an important role, at least at the beginning of shock expansion. Nevertheless, the description in Sect. 5 of the gain layer as being a "radiation-dominated" region remains justified, although in a generalized sense. While in the region around the neutrinosphere baryons (and possibly degenerate electrons) yield the major contribution to the pressure and internal energy, the importance of electronpositron pairs and photons increases at lower densities. In Sect. 5.2 (Eqs. (56)-(58)) it was argued that for $r>R_{\text {eos }}$ both the pressure contributions from relativistic and nonrelativistic particles can be written as $P \propto T^{4}$, provided that the electron fraction $Y_{\mathrm{e}}$ and the electron degeneracy parameter $\eta_{\mathrm{e}}$ do not vary strongly. In the gain layer this is fulfilled, because the electron degeneracy is typically small, i.e., $\eta_{\mathrm{e}} \lesssim \pi$, and electron-positron pairs are abundant (see the detailed discussion by Bethe 1993, 1996b). Indeed, the hydrodynamical simulation of Rampp \& Janka (2000) shows that $\eta_{\mathrm{e}}$ in the gain layer changes only between about 1.5 and 3 during the interesting phase of the post-bounce evolution.

Despite of the considerable contribution to the pressure which is provided by nonrelativistic baryons, also the use of $\Gamma=(\partial \ln P / \partial \ln \rho)_{\mathrm{s}}=\frac{4}{3}$ for the adiabatic index of the equation of state in the postshock region is justified, although the calculations in this paper are not constrained to this specific choice. At the conditions present between the gain radius and the shock (density between a few $10^{8} \mathrm{~g} \mathrm{~cm}^{-3}$ and several $10^{9} \mathrm{~g} \mathrm{~cm}^{-3}$ and temperatures between roughly $\lesssim 1 \mathrm{MeV}$ and $\gtrsim 2 \mathrm{MeV}$ ), a finite mass fraction of $\alpha$ particles is still present $\left(X_{\alpha} \lesssim 0.5\right)$. The disintegration of these $\alpha$ 's at nuclear statistical equilibrium around $1-1.5 \mathrm{MeV}$ and the growing importance of $\mathrm{e}^{+} \mathrm{e}^{-}$pairs and photons for higher temperatures produce $\Gamma$ values between 1.3 and 1.4. This can, for example, be verified by an inspection of the equation of state of Lattimer \& Swesty (1991).

\subsubsection{Steady-state conditions}

Steady-state conditions are realized when $\dot{R}_{\mathrm{s}}=\dot{R}_{\mathrm{g}}=0$, which in general requires that $\mathrm{d}\left(\Delta M_{\mathrm{g}}\right) / \mathrm{d} t=\mathrm{d}\left(\Delta E_{\mathrm{g}}\right) / \mathrm{d} t=$ 0. From Eq. (122) one gets $\dot{M}^{\prime}=\dot{M}$, which yields

$L_{\mathrm{acc}}=\dot{M}\left(l_{\nu}+q_{\mathrm{d}}\right)+\mathcal{H}-\mathcal{C}$,

when Eqs. (93) and (94) and the definition $l=(e+$ $P) / \rho-G \widetilde{M} / r$ are used (note that $\frac{1}{2} \rho v^{2} \ll \varepsilon$ at the neutrinosphere). Neutrino heating and cooling in the gain layer scale with $L_{\nu}\left(R_{\mathrm{g}}\right)$ and thus $L_{\nu}\left(R_{\nu}\right)$ (Eq. (76)), and depend on $R_{\mathrm{s}}$, directly as well as via $R_{\mathrm{g}}$. Also $L_{\text {acc }}$ depends on the neutrinospheric luminosity $L_{\nu}$ (Eq. (77)). Equation (121), on the other hand, yields

$\dot{M}=\frac{\mathcal{H}-\mathcal{C}}{l_{\mathrm{s}}-l_{\mathrm{g}}}$

for $l_{\mathrm{s}}<l_{\mathrm{g}}$, which is fulfilled if $\Gamma<\gamma$. $[\Gamma=\gamma$ requires $\mathcal{H}-\mathcal{C}=0$ and therefore $R_{\mathrm{g}}=\min \left(R_{\mathrm{s}}, R_{\alpha}\right)$.] From the combined Eqs. (131) and (132) the values of the shock radius $R_{\mathrm{S}}$ and the neutrinospheric luminosity $L_{\nu}$ can be determined which correspond to steady-state conditions for a given value of $\dot{M}$. The solution for $L_{\nu}$ lies on the critical $\mathrm{O}_{\mathrm{M}}$ line displayed in Figs. 4 and 5, where $\dot{M}^{\prime}$ and $\dot{M}$ are equal. The core luminosity has to satisfy this constraint, because the neutrinospheric conditions and the temperature in the cooling layer above the neutrinosphere are assumed to be regulated by the interaction with the high neutrino fluxes from the hot, neutrino-opaque neutron star. This inner boundary condition differs from the one used for discussing ordinary steady-state accretion onto neutron stars (Chevalier 1989). There the temperature of the optically thin medium at the base of the atmosphere is assumed to adopt a value which ensures that 
photon or neutrino losses carry away the binding energy of the matter which is accreted at a given constant rate. This requirement then yields a condition for calculating the steady-state position of the accretion shock.

\subsection{Convective energy transport}

The simplified analytic model described in this paper can certainly not account for the detailed effects associated with convective overturn in the neutrino-heated layer between gain radius and shock. This overturn is an intrinsically multi-dimensional phenomenon where low-entropy downflows and hot, rising bubbles of neutrino-heated gas coexist in the same region of the star. Therefore the mixing achieved by the gas motions is not complete even on a macroscopic scale. Nevertheless, some consequences and fundamental effects associated with the existence of convective energy transport in the gain region can be figured out.

The described analytic model distinguishes between the adiabatic index $\Gamma$ of the equation of state in the gain layer, and the structural polytropic index $\gamma$. The gain layer is subject to non-adiabatic changes, because energy deposition by neutrino heating takes place. Also, the gain layer is not necessarily isentropic. Using the developed framework of equations with $P=K \rho^{\gamma}, \gamma=\Gamma=$ const., and $K=$ const. for the equation of state in the gain region which is the default setting for the analyses in Sect. 9 -, Eq. (112) yields the same value for the total specific energies at $R_{\mathrm{g}}$ and $R_{\mathrm{s}}$. For a gas with adiabatic index $\Gamma$ this also means that isentropic conditions are realized. This, therefore, corresponds to the case where convection is very efficient in carrying energy from the gain radius, close to which neutrino heating is strongest, to directly behind the shock. Chosing instead $\gamma>\Gamma$ yields $l_{\mathrm{g}}>l_{\mathrm{s}}$, a result which is more characteristic of the situation without convection. Here the energy deposition by neutrinos establishes negative gradients of the entropy and specific energy between gain radius and shock.

Repeating the derivations of Sects. 5-9 with $\gamma>\frac{4}{3}$, reveals, on the one hand, that the gain radius $R_{\mathrm{g}}$ is smaller and therefore the optical depth and the net heating in the gain region, $\mathcal{H}-\mathcal{C}$, are somewhat larger than for the "standard" case of $\gamma=\frac{4}{3}$ (because the hydrostatic density and temperature profiles are flatter behind the shock). On the other hand, however, a less efficient energy transport from the gain radius to the shock has a severe disadvantage: The gas which is advected inward through $R_{\mathrm{g}}$, carries away a large fraction of the energy absorbed from neutrinos before. In Eq. (121) the term $\dot{M}^{\prime} l_{\mathrm{g}}$ yields a smaller positive or even a negative contribution when $\dot{M}^{\prime}<0$ and $l_{\mathrm{g}}$ is negative or positive, respectively. This reduces the net effect of neutrino heating and is harmful for shock expansion and acceleration.

A comparison of Figs. 6 and 7 demonstrates the differences. The time-dependent solutions for shock radius and specific energy in the gain layer were obtained with $\Gamma=\frac{4}{3}$ in both cases, but in Fig. $7 \gamma=1.45$ was chosen instead of $\gamma=\frac{4}{3}$. For $\gamma>\Gamma$ the shock expansion is weaker and the specific energy in the gain layer stays lower. The effect is particularly obvious for the core neutrino luminosity of $L_{\nu}=410^{52} \mathrm{erg} \mathrm{s}^{-1}$. Figure 6 shows a marginal success for this case, whereas in Fig. 7 the shock expansion fails.

These findings are confirmed by an inspection of the spherically symmetric simulation of the collapse and postbounce evolution of a $15 M_{\odot}$ progenitor star published recently by Rampp \& Janka (2000). After an expansion to more than $350 \mathrm{~km}$, the shock in this model finally recedes to a much smaller radius and fails to produce an explosion. The shock recession is caused (or accompanied) by a rapid decrease of the mass in the gain region, because more matter is flowing through the gain radius than is resupplied by accretion through the shock. In the hydrodynamical simulation one finds that $\Delta E_{\mathrm{g}}$ also decreases during this phase, an effect which should not occur if $l_{\mathrm{g}}=l_{\mathrm{s}}<0$ (compare Eq. (121)).

This discussion emphasizes the importance of convective energy transport between the gain radius and the shock. Postshock convection reduces the mass loss as well as the energy loss from the gain region, which are associated with the continuous inward advection of neutrinoheated gas during the phase of shock revival. Also an increase of the core luminosity can diminish the accretion of gas into the neutron star by suppressing the net neutrino emission from the cooling layer. Both effects have been demonstrated in numerical simulations to be helpful for an explosion.

\subsection{Limits of the toy model}

Employing idealized and in many respects simplifying assumptions, a toy model was developed here on grounds of an approximate solution of the hydrodynamic equations, Eqs. (2)-(4). By reducing the complexity, hopefully without sacrificing fundamental properties, the model is intended to help discussing the principles and the essence of the neutrino-driven mechanism. It is, however, not meant to compete with detailed hydrodynamical simulations, where usually a lot more refinements concerning the description of the stellar fluid and of the neutrino transport are included.

The stellar structure outside of the forming neutron star at the center is considered to consist of three layers: The cooling layer, from where neutrino loss extracts energy, is bounded by the neutrinosphere on the one side and the gain radius on the other; the heating layer extends between gain radius and shock and receives net energy deposition by neutrinos; in the infall region exterior to the shock, matter of the progenitor star moves inward at a significant fraction of the free-fall velocity. The evolution of the shock depends on the conditions in the gain layer. Assuming the radial structure of this layer to be given by hydrostatic equilibrium one can discuss the behavior of the shock in response to integral properties of the 
heating region. The total mass and energy of the gain layer change due to inflow and outflow of gas, neutrino heating, and a possible shift of the boundaries, and are therefore sensitive to the rate of mass accretion by the shock on the one hand, and the irradiation by the neutrinospheric luminosities on the other.

\subsubsection{Temperature in the cooling layer}

The present work concentrates on a discussion of the phase of shock revival due to neutrino energy deposition, where the infall of the stellar gas is inverted to outflow. This implies that the gas flow through the gain layer cannot be stationary, i.e., the mass infall rate into the shock, $\dot{M}$, is in general different from the mass accretion rate $\dot{M}^{\prime}$ by the nascent neutron star. Here $\dot{M}^{\prime}$ in dependence of the physical conditions near the neutron star surface is estimated by assuming that the temperature at and just outside the neutrinosphere is governed by the interaction of the stellar medium with the neutrinos streaming out from deeper layers. This temperature determines the energy loss from the cooling layer around the neutron star, the efficiency of which then drives the mass exchange (inflow or outflow) with the gain layer farther out.

This picture is certainly a simplification of the real situation. For example, when outward motion of the postshock gas sets in, the advection of gas through the gain radius may be quenched as found in spherically symmetric simulations. In the described model this effect could be reproduced if the temperature in the cooling layer would drop. The current set of equations, however, does not allow one to calculate this effect because it does not include how the temperature in the cooling layer depends on the expansion or compression of the neutron star atmosphere. On the other hand, in the three-dimensional situation downflows and rising bubbles can coexist when convective overturn is present in the gain layer, as suggested by two-dimensional hydrodynamical simulations (Herant et al. 1994; Burrows et al. 1995; Janka \& Müller 1996). In this case accretion does not need to stop even when the shock is accelerated outward (Bethe 1993, 1995; however, Burrows et al. 1995 see a decrease of the neutrino luminosity associated with a reduced accretion rate when the explosion sets in). Convective overturn and thus accretion might in fact continue until the shock has reached a radius above $1000 \mathrm{~km}$ (Bethe 1997). It is not easy to estimate the fraction of the gas which stays in the gain layer relative to the part which is advected inward to the neutron star. The ansatz described here may be considered as a crude attempt to do so.

\subsubsection{Hydrostatic equilibrium}

The stellar structure in the gain layer was calculated as a solution of the equation of hydrostatic equilibrium. The latter is derived from Eq. (3) combined with Eq. (2). When the velocity-dependent terms can be neglected, this yields

$$
\frac{\partial v}{\partial t}+v \frac{\partial v}{\partial r}=\frac{\mathrm{d} v}{\mathrm{~d} t}=-\frac{1}{\rho} \frac{\partial P}{\partial r}-\frac{\partial \Phi}{\partial r}=0 .
$$

Hydrostatic equilibrium holds in the regime where the fluid velocity $v$ is much smaller than the local sound speed $c_{\mathrm{s}}$. This is well fulfilled when the postshock gas settles inward, and is also well fulfilled when it starts moving outward behind an accelerating shock. Of course, omitting the $v(\partial v / \partial r)=\partial\left(v^{2} / 2\right) / \partial r$ term from Eq. (133) implies that the discussed toy model will not reproduce the solutions for a stationary wind, which has a critical point where $v=c_{\mathrm{s}}$. This is not a handicap during the shock revival phase and the onset of shock expansion, because the gas behind the (slowly propagating) shock front moves (relative to the shock) with subsonic velocities. It means, however, that the treatment is not sufficiently general to follow the explosion to large radii and high shock velocities, which is the evolutionary phase when the expanding medium around the nascent neutron star forms a neutrinodriven wind. When the shock is far out and the gain layer has expanded by a large amount, the assumption of hydrostatic equilibrium therefore becomes inadequate and the corresponding structure of the gain layer is not sufficiently accurate any longer. Unless the gas velocity approaches $c_{\mathrm{s}}$, the modification of the stellar density and pressure profiles due to velocity effects is small. In fact, when the time-dependent calculations of Sects. 9.4 and 9.5 were terminated at $t=0.1$ seconds, the specific kinetic energy of the gas immediately behind the shock was only a minor fraction of the specific internal energy, at most about $20 \%$. The integral kinetic energy of the gain layer was even smaller compared to the total internal energy.

Considering hydrostatic conditions means that the fluid velocity is assumed not to be relevant for the structure of the neutron star atmosphere. In fact, an accretion or outflow velocity field in the gain layer was not derived (and was not of direct relevance for the discussion), although the toy model employs the mass accretion rates $\dot{M}$ and $\dot{M}^{\prime}$. The fluid velocity immediately above the shock is given by the infall velocity of the gas, and at the gain radius it must be equal to $v_{\mathrm{g}}=\dot{M}^{\prime} /\left(4 \pi R_{\mathrm{g}}^{2} \rho_{\mathrm{g}}\right)$. Different rates for the mass accretion into the shock and the mass flow through the gain radius imply that the mass of the gain layer can grow or drop due to active mass inflow or outflow (in addition to the motion of the shock and of the gain radius as the outer and inner boundaries, respectively, of the considered stellar shell). Therefore the conditions in the gain layer are non-stationary, i.e., $\partial \rho / \partial t=0$ cannot be true in general.

Deriving a velocity profile for the gain layer requires solving Eq. (2) with non-vanishing time derivative of the density and the lower boundary condition being given by $v\left(R_{\mathrm{g}}\right)=v_{\mathrm{g}}$. Doing so, the velocity jump at the shock is also influenced by the physical conditions around the gain radius. This is analogue to the postshock density, which is sensitive to the mass accumulated in the gain layer, 
and is similar to the postshock pressure, which varies with the integral value of the energy deposited by neutrinos in the gain layer. Mass and energy loss or gain thus affect the whole heating layer simultaneously. Assuming hydrostatic equilibrium implies that the physical state of the gas behind the shock front is coupled to the conditions at the gain radius, because the sound crossing timescale is considered to be small compared with all other relevant timescales of the problem. Therefore the RankineHugoniot relations for the density jump and the velocity jump at the shock front cannot be satisfied exactly, which reflects the approximative nature of the hydrostatic structure. The violation of the Rankine-Hugoniot conditions (for specific values of the EoS parameters), however, is usually small and the overall properties of the calculated solutions should be close to the true ones, in particular at some distance behind the shock front.

\subsubsection{Equation of state and convection}

The equation of hydrostatic equilibrium in the gain layer was solved assuming that gas pressure, density and temperature are related by $P=K \rho^{\gamma}=A(k T)^{4}$ (Eq. 56)) with $\gamma, K$ and $A$ being constants. As discussed in Sects. 5.2 and 9.4, this is reasonably well fulfilled in the gain layer, where the stellar gas consists of a mixture of relativistic electrons, positrons and photons and nonrelativistic nucleons and nuclei. On the one hand the electron degeneracy is low $\left(\eta_{\mathrm{e}} \lesssim \pi\right)$ and both $\eta_{\mathrm{e}}$ and the electron fraction $Y_{\mathrm{e}}$ do not vary strongly (the variation of $Y_{\mathrm{e}}$ is at most a factor of about two, roughly between 0.25 at the gain radius and 0.5 at the shock). For these reasons fermion captures on nucleons were ignored concerning their effect on the $Y_{\text {e }}$ profile in the gain layer. On the other hand, heavy nuclei are mostly disintegrated into free nucleons with some admixture of $\alpha$ particles. Therefore also the sum of the nuclear number fractions $\sum_{i} Y_{i}$ is roughly constant; in fact, $\sum_{i} Y_{i} \approx 1$ is a good approximation. Because there is a finite mass fraction of $\alpha$ particles, $X_{\alpha}=4 Y_{\alpha} \lesssim 0.5$, nuclear statistical equilibrium yields an adiabatic index of $\Gamma=1+P / \varepsilon \approx \frac{4}{3}$ for the equation of state at typical postshock conditions, although nucleons are responsible for a large fraction of the pressure. None of these assumptions, however, is rigorously fulfilled in the medium of the gain layer at all radii and at all times. But making use of these assumptions simplifies the discussion considerably because the equation of state can be treated analytically. Since the overall properties of the stellar gas are accounted for, it is very unlikely that the conclusions on the qualitative level of this paper are affected when more refinements and complications are added. This might change details, but should not modify the essence of the toy model.

The energy input to the gain layer by neutrino heating is accounted for in the model. This energy gain from neutrinos means that the changes in a fluid element are non-adiabatic. Therefore the structural polytropic index $\gamma$ can be different from the adiabatic index $\Gamma$ of the EoS. Varying $\gamma$ allows one to mimic additional processes which might affect the evolution and behavior of the gain layer. Chosing $\gamma=\Gamma=\frac{4}{3}$ implies that the gain layer is considered to be isentropic, i.e., the energy deposited by neutrinos is assumed to be efficiently (and instantaneously) redistributed such that the entropy is roughly equal everywhere and $l_{\mathrm{s}}=l_{\mathrm{g}}$ holds (Eq. (112)). Since neutrino heating is strongest near the gain radius, this means that energy has to be transported from smaller radii to positions closer to the shock. Such an effect is realized by the strong postshock convection seen in multi-dimensional hydrodynamic simulations (e.g., Herant et al. 1994; Burrows et al. 1995; Janka \& Müller 1996). Using $\gamma>\Gamma$ a situation is described where more of the deposited energy stays near the gain radius, corresponding to less efficient energy transport by convection. The toy model confirms that this has a negative influence on the possibility of shock expansion.

\subsubsection{Neutrino processes}

Processes different from $\nu_{\mathrm{e}}$ and $\bar{\nu}_{\mathrm{e}}$ absorption and emission by nucleons were not taken into account for the neutrino heating and cooling above the neutrinosphere. Both neutrino-electron/positron scattering and neutrinopair annihilation have much smaller reaction cross sections than the baryonic processes and are less efficient in transferring energy to the stellar medium. For this reason they do not play a crucial role for the explosion mechanism (Bethe \& Wilson 1985; Bethe 1990, 1993, 1995, 1997; Cooperstein et al. 1987).

Effects due to muon and tau neutrinos and antineutrinos $\left(\nu_{x}\right)$ were completely ignored in the discussions of this paper. Because muons and tau leptons cannot be produced in the low-density medium above the neutrinosphere, $\nu_{\mu}$ and $\nu_{\tau}$ do not interact with nucleons via charged-current reactions and therefore couple to the gas less strongly than electron neutrinos and antineutrinos. Energy exchange by neutral-current scatterings off nucleons contributes in shaping their emission spectra near the neutrinosphere (Janka et al. 1996; Burrows et al. 2000) and might also be relevant for the heating in the gain layer. Although the recoil energy transfer per scattering is reduced by a factor $\epsilon /\left(m c^{2}\right)$ relative to the absorption of neutrinos with energy $\epsilon$ ( $m$ is the nucleon mass), the cross sections of both processes are similar and all flavors of neutrinos and antineutrinos participate in the neutral-current reactions with neutrons as well as with protons. Using Eq. (10) for the nucleon scattering opacity and the mean energy exchange per reaction as given by Tubbs (1979), one can estimate the importance of nucleon scattering for the energy transfer to the medium relative to $\nu_{\mathrm{e}}$ and $\bar{\nu}_{\mathrm{e}}$ absorption as:

$$
\begin{aligned}
& \frac{Q_{\nu, \mathrm{sc}}^{+}}{Q_{\nu, \mathrm{abs}}^{+}} \sim \frac{\frac{1+3 \alpha^{2}}{16} 5\left(Y_{\mathrm{n}}+Y_{\mathrm{p}}\right)\left(\left\langle\epsilon_{\nu_{x}}^{3}\right\rangle-6 k T\left\langle\epsilon_{\nu_{x}}^{2}\right\rangle\right)}{\frac{1+3 \alpha^{2}}{4}\left\langle\epsilon_{\nu_{\mathrm{e}}}^{2}\right\rangle\left(Y_{\mathrm{n}}+2 Y_{\mathrm{p}}\right) m c^{2}} \\
& \sim \frac{15}{2} \frac{\left(k T_{\nu_{x}}\right)^{2}}{\left(k T_{\nu_{\mathrm{e}}}\right)^{2}} \frac{k T_{\nu_{x}}-k T}{m c^{2}} \sim 30 \frac{k T_{\nu_{x}}-k T}{m c^{2}}
\end{aligned}
$$


where $Q_{\nu \text {,abs }}^{+}$was taken from Eq. (28) and $T$ is the gas temperature. $T_{\nu_{\mathrm{e}}}$ and $T_{\nu_{x}}$ are the spectral temperatures of electron neutrinos and heavy-lepton antineutrinos, respectively, for which $T_{\nu_{\mathrm{e}}}^{2} \approx \frac{1}{2} T_{\bar{\nu}_{\mathrm{e}}}^{2} \approx \frac{1}{4} T_{\nu_{x}}^{2}$ was assumed again (compare Sect. 3). Moreover, the estimate was obtained by using $L_{\nu_{\mathrm{e}}} \approx L_{\bar{\nu}_{\mathrm{e}}} \approx L_{\nu_{x}}$ and $Y_{\mathrm{n}}+2 Y_{\mathrm{p}} \approx 1$. The spectral average of the third power of the neutrino energy, $\left\langle\epsilon_{\nu_{x}}^{3}\right\rangle$, was defined in analogy to Eq. (25). Since the spectral temperatures and therefore the scattering cross sections of $\nu_{\mathrm{e}}$ and $\bar{\nu}_{\mathrm{e}}$ are smaller than those of $\nu_{x}$, electron neutrinos plus antineutrinos were given roughly the same weight as one of the heavy-lepton neutrinos. For typical values $k T_{\nu_{x}} \sim 8 \mathrm{MeV}$ and $k T \sim 2 \mathrm{MeV}$ one therefore derives a relative contribution of scattering processes to the neutrino heating in the gain layer of about $20 \%$.

Apart from this moderate amplification of the heating, muon and tau neutrinos have other effects on the shock propagation during the post-bounce evolution of a supernova. Within the first tens of milliseconds after shock formation, muon and tau neutrino pairs are produced by $\mathrm{e}^{ \pm}$annihilation in the heated matter immediately behind the shock. In addition to the disintegration of nuclei and the emission of $\nu_{\mathrm{e}}$ and $\bar{\nu}_{\mathrm{e}}$, this extracts energy from the shock-heated layers and weakens the prompt bounce shock. Somewhat later, between several ten milliseconds and a few hundred milliseconds after bounce, most of the muon and tau neutrinos come from the hot mantle layer of accreted material below the neutrinosphere of the forming neutron star. Since $\nu_{\mu}$ and $\nu_{\tau}$ pairs now carry away energy which otherwise would be radiated in electron neutrinos and antineutrinos and would thus be more efficient for the heating behind the shock, this will have a negative effect on the possibility of shock rejuvenation. During the following phase of the evolution, when the deleptonization of the neutron star advances to deeper layers and the neutron star enters the Kelvin-Helmholtz cooling stage (Burrows \& Lattimer 1986), muon and tau neutrinos are mostly produced at higher densities. Being less strongly coupled to the nuclear medium, they diffuse to the surface more rapidly than $\nu_{\mathrm{e}}$ and $\bar{\nu}_{\mathrm{e}}$. This helps keeping the neutrinospheric layer hot, where electron neutrinos and antineutrinos take over a larger part of the energy transport. During this late phase of the evolution, $\nu_{\mu}$ and $\nu_{\tau}$ might thus even support higher $\nu_{\mathrm{e}}$ and $\bar{\nu}_{\mathrm{e}}$ fluxes.

\section{Summary and conclusions}

In this paper an analytic approach was presented which allows one to discuss the conditions for the revival of a stalled supernova shock by neutrino heating. The treatment is time-dependent in the sense that the gas flow is not assumed to be steady and the model can be used to calculate the shock radius, shock velocity, and the properties of the gain layer as functions of time.

\subsection{Components of the toy model}

The "atmosphere" of the collapsed stellar core outside of the neutrinosphere is considered to consist of three distinct layers (Sect. 2). Between neutrinosphere and gain radius there is a cooling region where neutrino emission extracts energy. In a heating layer between gain radius and shock, $\nu_{\mathrm{e}}$ and $\bar{\nu}_{\mathrm{e}}$ absorptions on nucleons dominate the inverse capture reactions of electrons and positrons and deposit energy. Finally, there is an infall region above the shock, where the gas of the progenitor star is accelerated to nearly the free-fall velocity. These different layers are in contact and exchange mass and energy.

The radial structure of the cooling and heating layers is assumed to be described by the conditions of hydrostatic equilibrium, which requires that the sound travel timescale is smaller than the other relevant timescales of the problem. This assumption is reasonably well fulfilled during the phase when the shock is near stagnation or just starts to gain momentum. For such conditions the layer behaves like one unit and reacts to changes in an infinitesimally short time. In combination with a simple representation of the equation of state, hydrostatic equilibrium allows one to calculate the density and pressure profiles analytically (Sect. 5). The radius and velocity of the shock then depend on integral properties of the gain layer, i.e., its total mass and energy.

Changes of the mass and energy integrals are caused by the motion of the boundaries or by active mass flow into or out of the gain layer. In Sect. 8 conservation equations for these global quantities were derived by integrating the equations of hydrodynamics, including the terms with time derivatives, over the volume of the gain layer. Following these integral quantities it was then possible to compute the shock position and shock velocity as well as other important quantities, e.g., the location of the gain radius, as functions of time. Assuming hydrostatic equilibrium thus reduces the mathematical problem to an integration of a set of ordinary differential equations with time as the independent variable. Discussing the destiny of the supernova shock therefore means solving an initial value problem. This expresses the fact that the shock evolution depends on the initial conditions, for example, on the shock stagnation radius and the initial energy in the gain layer, and is controlled by the cumulative effects of neutrino energy deposition and mass accumulation in the gain layer.

In general the gas falling through the shock will not move as a stationary flow between shock and neutrinosphere: the rate at which mass is advected through the gain radius is usually different from the mass accretion rate by the shock. Steady-state accretion or mass loss are special cases, which should be limits of the more general situation.

It is not easy to calculate the fraction of the accreted gas which stays in the gain layer. The neutron star can "swallow" matter only at a finite rate, depending on the efficiency with which the gas gets rid of the excess energy that prevents its integration into the neutron star surface. This efficiency is a sensitive function of the conditions in the cooling layer above the neutrinosphere. Since the hot, 
nascent neutron star below the neutrinosphere is a source of intense neutrino radiation and these neutrinos interact still frequently in the cooling region, the temperature there should be close to the neutrinospheric value. With this assumption and with known radius and density profile it is possible to calculate the neutrino energy loss from the cooling layer. This then allows one to derive a rough estimate for the rate at which gas can be advected through the neutrinosphere into the neutron star (Sect. 7).

Up to this stage the discussion does not require a detailed solution for the velocity field of the flow. Since hydrostatic conditions are assumed to hold, in which case the kinetic energy is small compared to internal and gravitational energies, and the time evolution can be discussed by considering integral quantities, it is sufficient to know the rates at which mass enters or leaves the gain layer at both boundaries.

The model described here is based on a number of simplifications and approximations. With the analytic representation of the equation of state developed in Sect. 5.2, there is no need to monitor the radial profile and time evolution of the electron fraction. In addition to assuming hydrostatic equilibrium this, of course, limits the accuracy of the radial structure derived for the gain layer. Other shortcomings are the treatment of the neutron star as a point mass, i.e., the gravity of the atmosphere above the neutrinosphere is neglected, general relativistic effects are ignored, the energy release by nucleon recombination in the postshock medium at $k T \lesssim 1-2 \mathrm{MeV}$ is not included, and additional neutrino heating and cooling by neutrinoelectron scattering and neutrino-pair processes are not considered. Although it may be desirable to include these effects for a more detailed solution, it seems very unlikely that more refinements will change the essence of the discussion.

In calculating the neutrino energy deposition in the gain layer the neutrinospheric luminosity as well as the neutrino emission from the cooling layer, which is associated with the accretion of gas onto the neutron star, are taken into account. The most problematic and probably most serious weakness of the presented toy model, however, is the overly simplified description of the conditions in the cooling layer, which are essential for estimating both the mass accretion rate and the accretion luminosity of the neutron star. The temperature of the medium around the neutrinosphere is certainly not only determined by the interaction with the neutrino flow from the neutrinosphere, but also depends on the processes in contact with the gain layer. This is currently not included in the toy model.

Despite of these simplifications the toy model yields interesting insights into the interdependence of effects and processes which determine the post-bounce evolution of the supernova shock. Thus it may help one understanding the results of the much more complex hydrodynamic simulations.

\subsection{Results and conclusions}

In particular, the discussion of this paper allows one to draw the following conclusions:

1. A criterion for shock revival could be derived in Sects. 9.1 and 9.2. It defines the conditions for which the radius $R_{\mathrm{s}}$ and the velocity $U_{\mathrm{s}}=\dot{R}_{\mathrm{s}}$ of the supernova shock can grow simultaneously. This criterion, applied to a stalled shock $\left(U_{\mathrm{s}} \sim 0\right)$, states that expansion $\left(\dot{R}_{\mathrm{s}}>0\right)$ and acceleration $\left(\dot{U}_{\mathrm{s}}>0\right)$ will occur at the same time when both the mass and the energy in the gain layer increase, i.e., when $\mathrm{d}\left(\Delta M_{\mathrm{g}}\right) / \mathrm{d} t>0$ and $\mathrm{d}\left(\Delta E_{\mathrm{g}}\right) / \mathrm{d} t>0$

2. For fixed neutrinospheric radius and temperature and given neutron star mass, the destiny of a shock at radius $R_{\mathrm{s}}$ depends on the rate at which the shock accretes matter, $\dot{M}$, and on the $\nu_{\mathrm{e}}$ plus $\bar{\nu}_{\mathrm{e}}$ luminosity $L_{\nu}$ coming from the neutrinosphere. In the $\dot{M}-L_{\nu}$ plane the two inequality relations of the shock revival criterion define two critical lines, an upper one and a lower one, which enclose the region where favorable conditions for shock expansion and acceleration occur. Neutrino heating is strong enough there to ensure $\mathrm{d}\left(\Delta E_{\mathrm{g}}\right) / \mathrm{d} t>0$ for a growing mass of the gain layer. The existence of a threshold value of the core neutrino luminosity (Burrows \& Goshy 1993) for a given value of $\dot{M}$ is confirmed. Besides stronger neutrino heating in the gain layer, the main effect of a higher $L_{\nu}$ is reducing the neutrino emission in the cooling region and thus suppressing the rate at which mass is advected into the neutron star. If $L_{\nu}$ drops below the threshold value, the gain layer loses more mass than it receives by gas falling into the shock. Therefore the pressure support for the shock breaks down and the shock retreats. The same effect can be caused when the mass accretion rate $\dot{M}$ drops below a critical limit. This means that not only a low core luminosity but also a low mass accretion rate by the shock can prevent shock expansion;

3. The efficiency of the neutrino cooling between neutrinosphere and gain radius is an important factor which contributes to regulating the mass advection through the gain radius and into the neutron star, and thus affects also the evolution of the gain layer and of the shock. Although the description in the toy model is greatly simplified, it demonstrates the importance of an accurate treatment of the physics, in particular of the neutrino-matter interactions, in the cooling layer. It must be suspected that excessive neutrino emission in the cooling layer, causing mass and energy loss from the gain layer, and not insufficient neutrino heating in the gain layer, may have been the main reason why spherically symmetric simulations ultimately failed to produce explosions, although the shock had expanded to larger radii, at least for some period of the postbounce evolution (see, e.g., Bruenn 1993; Bruenn et al. 1995; Rampp \& Janka 2000); 
4. The area between the two critical lines in the $\dot{M}-L_{\nu}$ plane grows for larger shock radii, because the conditions for neutrino heating in the gain layer improve. For parameters $\left(|\dot{M}|, L_{\nu}\right)$ above the upper critical line, neutrino energy deposition cannot compensate for the inflow of "negative total energy" with the gas that is falling through the shock. Since the mass in the gain layer increases, the shock nevertheless expands. But only after the shock has reached a sufficiently large radius can neutrino heating raise the total energy in the gain layer. When this radius is very far out, the total energy in the gain layer may stay negative, even for high neutrino luminosities, because only a small fraction of the gas in the gain layer experiences favorable heating conditions. In this case an explosion cannot occur. For the parameters considered in the discussed model, this happens when the (absolute value of the) mass accretion rate by the shock is larger than about $4 M_{\odot} \mathrm{s}^{-1}$. Also for somewhat lower accretion rates and in cases where the energy in the gain layer has become positive, explosions might not be possible. The question whether the shock is finally able to eject some part of the mantle and envelope of the progenitor star requires a global treatment of the problem. The toy model developed in this paper, however, is suitable to discuss only the phase of shock revival in dependence of the conditions around the forming neutron star. Considering the early evolution of the supernova shock is in general not sufficient to make predictions about the final outcome, in particular in cases where the postshock medium cannot acquire enough energy to gravitationally unbind the whole mass above the gain radius;

5 . The equations of the toy model were integrated for time-dependent solutions $R_{\mathrm{s}}(t)$ and $U_{\mathrm{s}}(t)$, and characteristic properties of the gain layer as functions of time. These solutions confirm the conclusions drawn from an evaluation of the shock revival criterion. In addition, they yield information about the evolution of the mass and energy in the gain layer;

6. Accounting for the complex effects of multidimensional convective overturn within the simplified discussion of the toy model is not possible. Nevertheless, some consequences of convective energy transport in the gain layer can be addressed. For a suitable choice of the structural polytropic index $\gamma$ of the hydrostatic atmosphere one can describe a situation where the gain layer is essentially isentropic in contrast to a case where the energy transport by convection is less efficient and therefore negative gradients of the entropy and specific energy are present between the gain radius and the shock front. Without convection such negative gradients must develop because neutrino heating is strongest just outside of the gain radius. Despite of a slightly larger neutrino energy deposition (because of a smaller gain radius) the absence of "convection" in the toy model has a negative effect on the shock expansion. Because convection redistributes the energy deposited by neutrinos mainly near the gain radius to regions closer to the shock, the energy loss associated with the downward advection of gas through the gain radius is reduced. Therefore more energy stays in the gain layer, in particular at larger radii, the postshock pressure is enhanced, and the shock is driven out more easily;

7. Parametric studies with the toy model suggest that successful explosions, driven by neutrino energy deposition, cannot be very energetic. The total energy per unit mass (gravitational plus internal energy plus minor kinetic contributions) in the expanding gain layer (between gain radius and shock) was observed to saturate around $0.1 \mathrm{~s}$ after shock revival and was found to be always limited, even for high core luminosities $L_{\nu}$, by $\Delta E_{\mathrm{g}} / \Delta M_{\mathrm{g}} \lesssim 10^{52} \operatorname{erg} M_{\odot}^{-1}$, (corresponding to an energy per nucleon of $\$ 5 \mathrm{MeV}$ ). For "typical" mass accretion rates by the shock, $\dot{M}$, of a few $0.1 M_{\odot} \mathrm{s}^{-1}$, the integral mass in the gain layer was then between several $10^{-2} M_{\odot}$ and (1-2) $10^{-1} M_{\odot}$, the corresponding total energy in the gain layer at most around $10^{51} \mathrm{erg}$. For higher accretion rates $\dot{M}$ the mass in the gain layer was found to be larger, but the energy per nucleon at the same time lower. The maximum total energies were obtained for intermediate values of $\dot{M}$ (around $1 M_{\odot} \mathrm{s}^{-1}$ ) and high $\nu_{\mathrm{e}}$ plus $\bar{\nu}_{\mathrm{e}}$ luminosities $L_{\nu}$ (values up to $1210^{52} \mathrm{erg} \mathrm{s}^{-1}$ were considered), but $\Delta E_{\mathrm{g}}$ was less than $(2-3) 10^{51} \mathrm{erg}$ in all cases.

\subsection{Implications}

The physical mechanism of powering the explosion by neutrino absorption on nucleons therefore seems to limit the explosion energy to values of at most a few $10^{51} \mathrm{erg}$. There is no obvious reason why neutrino-driven explosions could not be less energetic. The upper limit of the explosion energy is of the order of or a small multiple of the gravitational binding energy of the gas mass in the neutrinoheating layer around the nascent neutron star.

The reason for this energy limit is a very fundamental one, associated with the mechanism how the energy for the explosion is delivered, stored and carried outward. The energy which starts and drives the explosion is mainly transferred to the stellar gas by electron neutrino and antineutrino absorption on nucleons. As soon as the baryons have obtained a sufficiently large mean energy the expansion of the heating region sets in and the nucleons move away from the central source of the neutrino flux. The specific energy for this to happen is of the order of the gravitational binding energy of a nucleon. In fact, because of the inertia of the gain layer and the confinement by the matter falling into the shock, the nucleons can absorb more energy than that. If this were not the case, the energy of the expanding layer would be consumed by lifting the baryons in the gravitational field of the neutron star, and the kinetic energy at infinity could never be large. 
The neutrinospheric luminosity $L_{\nu}$ as well as the mass in the gain layer behind a stalled shock decrease with time, associated with the decreasing rate $\dot{M}$ of mass accretion by the shock. This has negative effects on the possibility of shock revival, as discussed above on grounds of the toy model. It definitely does not improve the conditions for a strong explosion, because of the limited energy which a baryon can absorb before it starts moving outward. With little gas being exposed to neutrino heating the explosion energy will therefore stay low. For these reasons "late" neutrino-driven explosions appear to be disfavored compared to delayed ones that develop within the post-bounce period when both $L_{\nu}$ and $\dot{M}$ are still high. This is typically the case until about half a second after core bounce.

Estimating the final explosion energy of the star requires, of course, that the energy release by nucleon recombination and possible nuclear burning in a fraction of the mass of the gain layer are added, and the gravitational binding energy of the mantle and envelope material of the progenitor star is subtracted (see, e.g., Bethe 1990, 1993; Bethe 1996a,c). Within the considered toy model these energies cannot be estimated. In case of a successful neutrino-driven explosion these terms, however, should not be the dominant ones in the total energy budget.

The total energy of the explosion should also not receive a major contribution by the energy released during the phase of the neutrino-driven wind, which succeeds the period of shock revival and early shock expansion. Different from the latter phase, the neutrino-driven wind is characterized by quasi steady-state conditions, with the mass flow rate not varying with the radius outside of a narrow region where the mass loss of the neutron star is determined. Baryons interacting with neutrinos near the surface of the neutron star cannot absorb a particle energy much larger than their gravitational binding energy before they are driven away from the neutrinosphere. Although always positive, the neutrino heating decreases rapidly when the wind accelerates outward and the distance from the source of the luminosity increases. Since the confining effect of mass infall to a shock is absent, the final net energy of a nucleon moving out with the wind will be even smaller than at earlier times. In addition, the neutrino luminosity and the neutron star radius shrink with time. Therefore the mass loss rate during the wind phase will be lower than right after shock revival. For these reasons the total mass ejected in the wind is expected to be less than a few $10^{-2} M_{\odot}$ (see Woosley \& Baron 1992; Woosley 1993a; Qian \& Woosley 1996).

Overcoming the stringent limit on the energy per nucleon that neutrinos can transfer to the heated matter, requires specific conditions. It could either be achieved by a sudden, luminous outburst of (energetic) neutrinos, which builds up on a timescale shorter than the expansion time of the gas around the neutron star. However, assuming a standard, hydrostatic cooling history of the nascent neutron star, there is no theoretical model to support such a scenario. Alternatively, the energy of the explosion could be absorbed and carried by non-baryonic particles, i.e., electrons and positrons and photons. In both cases the total energy is not constrained roughly by the binding energy of the gas in the gravitational potential of the neutron star. In fact, neutrino-electron scattering and neutrino-antineutrino annihilation have been suggested as important sources of energy for the explosion (Goodman et al. 1987; Colgate 1989). An accurate discussion of the physics of neutrino transport in the semi-transparent regime around the neutrinosphere (Janka 1991a,b) and a detailed evaluation of the conditions in the heating layer, however, show that both $\nu \mathrm{e}^{ \pm}$scattering (Bethe \& Wilson 1985; Bethe 1990, 1993, 1995) and $\nu \bar{\nu}$ annihilation (Cooperstein et al. 1987; Bethe 1997) are significantly less efficient than $\nu_{\mathrm{e}}$ and $\bar{\nu}_{\mathrm{e}}$ absorption, and thus contribute only minor fractions to the explosion energy.

The situation may be different when the global spherical symmetry is broken, e.g., in case of a black hole that accretes gas from a thick disk formed by the collapsing matter of a rapidly rotating, massive star. The disk becomes very hot and loses energy primarily by neutrino emission. Such a scenario was suggested as source of cosmological gamma-ray bursts and possibly strange, very energetic supernova explosions (Woosley 1993b; MacFadyen \& Woosley 1999; MacFadyen et al. 1999). In this case the neutrino luminosities can be higher, the region where neutrino pairs annihilate is more compact (which implies that the neutrino number densities are larger), and the geometry favors more head-on collisions between neutrinos. All these effects lead to an enhanced probability of $\nu \bar{\nu}$ annihilation in the close vicinity of the black hole (Popham et al. 1999).

Acknowledgements. It is a pleasure to thank M. Rampp for comments, his patience in many discussions, and a comparative evaluation of his spherically symmetric hydrodynamical simulations. The author is very grateful to an anonymous referee for thoughtful and knowledgable comments which helped to improve the manuscript significantly. The preparation of Fig. 2 by Mrs. H. Krombach is acknowledged as well as help by M. Bartelmann to tame the "LATEX devil". This work was supported by the SFB-375 "Astroparticle Physics" of the Deutsche Forschungsgemeinschaft.

\section{References}

Baron, E., \& Cooperstein, J. 1990, ApJ, 353, 597

Bethe, H. A. 1990, Rev. Mod. Phys., 62, 801

Bethe, H. A. 1993, ApJ, 412, 192

Bethe, H. A. 1995, ApJ, 449, 714

Bethe, H. A. 1996a, ApJ, 469, 737

Bethe, H. A. 1996b, ApJ, 473, 343

Bethe, H. A. 1996c, Nucl. Phys. A, 606, 95

Bethe, H. A. 1997, ApJ, 490, 765

Bethe, H. A., \& Wilson, J. R. 1985, ApJ, 295, 14

Bloom, J. S., et al. 1999, Nature, 401, 453

Bludman, S. A., \& Van Riper, K. A. 1978, ApJ, 224, 631

Brown, G. E., \& Weingartner, J. C. 1994, ApJ, 436, 843

Bruenn, S. W. 1985, ApJS, 58, 771

Bruenn, S. W. 1986a, ApJ, 311, L69

Bruenn, S. W. 1986b, ApJS, 62, 331

Bruenn, S. W. 1989a, ApJ, 340, 955 
Bruenn, S. W. 1989b, ApJ, 341, 385

Bruenn, S. W. 1993, in Nuclear Physics in the Universe, ed. M. W. Guidry, \& M. R. Strayer (Institute of Physics Publ., Bristol), 31

Bruenn, S. W., Mezzacappa, A., \& Dineva, T. 1995, Phys. Rep., 256, 69

Burrows, A. 1987, ApJ, 318, L57

Burrows, A., \& Goshy, J. 1993, ApJ, 416, L75

Burrows, A., \& Lattimer, J. M. 1986, ApJ, 307, 178

Burrows, A., \& Sawyer, R. F. 1998, Phys. Rev. C, 58, 554

Burrows, A., \& Sawyer, R. F. 1999, Phys. Rev. C, 59, 510

Burrows, A., Hayes, J., \& Fryxell, B. A. 1995, ApJ, 450, 830

Burrows, A., Young, T., Pinto, P. A., Eastman, R., \& Thompson, T. 2000, ApJ, 539, 865

Chevalier, R. A. 1989, ApJ, 346, 847

Colgate, S. A. 1989, Nature, 341, 489

Colgate, S. A., \& White, R. H. 1966, ApJ, 143, 626

Colgate, S. A., \& Fryer, C. 1995, Phys. Rep., 256, 5

Colgate, S. A., Herant, M., \& Benz, W. 1993, Phys. Rep., 227, 157

Cooperstein, J., Bethe, H. A., \& Brown, G. E. 1984, Nucl. Phys. A, 429, 527

Cooperstein, J., van den Horn, L. J., \& Baron, E. A. 1987, ApJ, 321, L129

Fryer, C. L. 1999, ApJ, 522, 413

Fryer, C. L., Benz, W., \& Herant, M. 1996, ApJ, 460, 801

Galama T. J., et al. 1998, Nature, 395, 670

Goodman, J., Dar, A., \& Nussinov, S. 1987, ApJ, 314, L7

Herant, M., Benz, W., Hix, W. R., Fryer, C. L., \& Colgate, S. A. 1994, ApJ, 435, 339

Hillebrandt, W. 1987, in High Energy Phenomena Around Collapsed Stars, ed. F. Pacini (Reidel, Dordrecht), 73

Höflich, P., Wheeler, J. C., \& Wang, L. 1999, ApJ, 521, 179

Iwamoto, K, et al. 1998, Nature, 395, 672

Janka, H.-Th. 1991a, Ph.D. Thesis, TU München, MPAReport, 587

Janka, H.-Th. 1991b, A\&A, 244, 378

Janka, H.-Th. 1992, A\&A, 256, 452

Janka, H.-Th. 1995, Astropart. Phys., 3, 377

Janka, H.-Th., \& Müller, E. 1995, ApJ, 448, L109

Janka, H.-Th., \& Müller, E. 1996, A\&A, 306, 167

Janka, H.-Th., Keil, W., Raffelt, G., \& Seckel, D. 1996, Phys. Rev. Lett., 76, 2621

Keil, W., Janka, H.-Th., \& Müller, E. 1996, ApJ, 473, L111

Khokhlov, A. M., Höflich, P. A., Oran, E. S., et al. 1999, ApJ, 524, L107

Lattimer, J. M., \& Swesty, F. D. 1991, Nucl. Phys. A, 535, 331

Lichtenstadt, I., Khokhlov, A. M., \& Wheeler, J. C. 1999, preprint, submitted to ApJ

Liebendörfer, M., Mezzacappa, A., Thielemann, F.-K., Messer, O. E. B., Hix, W. R., \& Bruenn, S. W. 2000, preprint [astro-ph/0006418]

MacFadyen, A., \& Woosley, S. E. 1999, ApJ, 524, 262

MacFadyen, A., Woosley, S. E., \& Heger, A. 1999, ApJ, in press [astro-ph/9910034]
Mayle, R., \& Wilson, J. R. 1988, ApJ, 334, 909

Messer, O. E. B., Mezzacappa, A., Bruenn, S. W., \& Guidry, M. W. 1998, ApJ, 507, 353

Mezzacappa, A., Calder, A. C., Bruenn, S. W., et al. 1998a, ApJ, 493, 848

Mezzacappa, A., Calder, A. C., Bruenn, S. W., et al. 1998b, ApJ, 495, 911

Mezzacappa, A., Liebendörfer, M., Messer, O. E. B., et al. 2000, Phys. Rev. Lett., submitted [astro-ph/0005366]

Myra, E. S., \& Bludman, S. A. 1989, ApJ, 340, 384

Myra, E. S., Bludman, S. A., Hoffman, Y., Lichtenstadt, I., Sack, N., \& Van Riper, K. A. 1987, ApJ, 318, 744

Qian, Y.-Z., Woosley, S. E. 1996, ApJ, 471, 331

Pons, J. A., Reddy, S., Prakash, M., Lattimer, J. M., \& Miralles, J. A. 1999, ApJ, 513, 780

Popham, R., Woosley, S. E., \& Fryer, C. 1999, ApJ, 518, 356

Raffelt, G. G., \& Seckel, D. 1995, Phys. Rev. D, 52, 1780

Rampp, M. 2000, Ph.D. Thesis, TU München

Rampp, M., \& Janka, H.-Th. 2000, ApJ, 539, L33

Reddy, S., Prakash, M., \& Lattimer, J. M. 1998, Phys. Rev. D, 58, 013009

Reddy, S., Prakash, M., Lattimer, J. M., \& Pons, J. A. 1999, Phys. Rev. C, 59, 2888

Rybicki, G. B., \& Lightman, A. P. 1979, Radiative Processes in Astrophysics (John Wiley \& Sons, New York)

Shapiro, S. L., \& Teukolsky, S. A. 1983, Black Holes, White Dwarfs, and Neutron Stars (John Wiley \& Sons, New York)

Shigeyama, T. 1995, Publ. Astron. Soc. Jan., 47, 581

Suzuki, H. 1989, Neutrino Burst from Supernova Explosion and Proto Neutron Star Cooling, Ph.D. Thesis, Univ. Tokyo

Takahashi, K., El Eid, M. F., \& Hillebrandt, W. 1978, A\&A, 67,185

Thompson, C. 2000, ApJ, 534, 915

Tubbs, D. L. 1979, ApJ, 231, 846

Wang, L., \& Wheeler, J. C. 1998, ApJ, 504, L87

Wilson, J. R. 1985, in Numerical Astrophysics, ed. J. M. Centrella, J. M. LeBlanc, \& R. L. Bowers (Jones and Bartlett Publ., Boston), 422

Wilson, J. R., \& Mayle, R. 1988, Phys. Rep., 163, 63

Wilson, J. R., \& Mayle, R. 1993, Phys. Rep., 227, 97

Wilson, J. R., Mayle, R., Woosley, S. E., \& Weaver, T. 1986, Ann. NY Acad. Sci., 470, 267

Woosley, S. E. 1993a, A\&AS, 97, 205

Woosley, S. E. 1993b, ApJ, 405, 273

Woosley, S. E., \& Baron, E. 1992, ApJ, 391, 228

Woosley, S. E., \& Weaver, T. A. 1994, in Supernovae, ed. S. A. Bludman, R. Mochkovitch, \& J. Zinn-Justin, North-Holland, Amsterdam, 63

Woosley, S. E., Eastman, R. G., \& Schmidt, B. P. 1999, ApJ, 516,788

Woosley, S. E., Wilson, J. R., \& Mayle, R. 1986, ApJ, 302, 19

Yamada, S. 2000, Nucl. Phys. A, 662, 219

Yamada, S., \& Toki, H. 2000, Phys. Rev. C, 61, 5803

Yamada, S., Janka, H.-Th., \& Suzuki, H. 1999, A\&A, 344, 533 